Shaffer et al. Metabolite-microbe profiles are shaped by the environment

\title{
Multi-omics profiling of Earth's biomes reveals that microbial and metabolite composition are shaped by the environment
}

\begin{abstract}
.
Using sequence data to understand function in microbial communities presents challenges, in part due to the complex intricacies of processes such as transcription and translation. Here, we sample the metabolome directly using untargeted metabolomics, and combine it with metagenomic sequencing to harness a multi-omics approach. We focus on a diverse sample set representing Earth's habitats to describe the distribution of metabolites, and their relationships with microbial communities and the environment.
\end{abstract}

Microbes produce an array of secondary metabolites that perform diverse functions from communication to defense ${ }^{1}$. These metabolites have been used to benefit human health and sustainability ${ }^{2}$. To begin to understand the metabolic capabilities of microbial communities, metagenomic sequence data can be profiled for genes related to certain products, allowing interpretation of metabolic potential ${ }^{3}$. Although metagenome mining is a powerful way to annotate biosynthetic gene clusters (BCGs), chemical evidence is required to confirm the presence of metabolites and comprehensively address this fundamental hypothesis, as metagenomic data only identify metabolic potential. To describe the Earth's metabolome, we use an integrated omics approach: the direct survey of metabolites associated with microbial communities spanning diverse environments using untargeted metabolomics coupled with metagenome analysis. We show that the presence of certain classes of secondary metabolites can be limited or amplified by the environment. Importantly, our data indicate that considering the relative abundances of secondary metabolites (i.e., rather than only presence/absence) strengthens differences in metabolite profiles across environments, and that their richness and 
Shaffer et al. Metabolite-microbe profiles are shaped by the environment

composition in any given sample do not directly reflect those of co-occurring microbial communities, but rather vary with the environment.

From a genomics perspective, information regarding metabolic potential is obtained through detection and classification of BGCs, the genomic loci underlying the production of secondary metabolites and their precursors ${ }^{4}$. The most sensitive approaches amplify BGCspecific marker sequences by $\mathrm{PCR}^{5}$, but only metagenomic methods can link BGCs to their genomes (i.e., metagenome-assembled genomes, or MAGs) of origin and detect BGCs in novel MAGs. Along these lines, a recent large-scale analysis of putative BGC regions from microbial MAGs surprisingly concluded that, although the main classes of secondary metabolites are enriched in particular microbial taxa, the relative distribution of secondary metabolite biosynthetic potential across environments was conserved, implying that most classes of secondary metabolites are not limited or amplified by the environment. One caveat of such analyses is that annotated BGCs often have incomplete sequences, potentially impacting annotation and quantification ${ }^{3}$. More importantly, gene-level data about BGCs inferred from MAGs cannot offer information about actual synthesis (e.g., gene expression), creating uncertainty about the distribution of secondary metabolites across environments ${ }^{6-9}$. Even with high-coverage gene expression data, currently lacking for most environments, the complex structural and modular nature of many secondary metabolites prevents their accurate association with the underlying genomic origins ${ }^{10}$. Furthermore, quantifying metabolite diversity from such metatranscriptomic and/or metaproteomic data (also lacking for most environments) is problematic due to a suite of post-translational processes that can dissociate the level of gene transcription from the abundance of gene products ${ }^{11}$. Finally, shotgun metagenomics does not 
Shaffer et al. Metabolite-microbe profiles are shaped by the environment

capture BGCs from low-abundance MAGs efficiently, as shown from comparative studies of targeted sequencing approaches ${ }^{5}$.

An approach to surmount these issues is to complement metagenomics with a direct survey of secondary metabolites using untargeted metabolomics. Liquid chromatography with untargeted tandem mass spectrometry (LC-MS/MS) is a versatile method that detects tens of thousands of metabolites in biological samples ${ }^{12}$. Although LC-MS/MS metabolomics has generally suffered from a low metabolite annotation rate when applied to non-model organisms, recent computational advances can systematically classify metabolites using their fragmentation spectra $^{13}$. Untargeted metabolomics provides the relative abundance (i.e., intensity) of each metabolite detected across samples rather than just counts of unique structures (e.g., Fig. 1a vs. 1b), and thus provides a direct readout of the surveyed environment, a result that is difficult to achieve with a purely genomics approach. Whereas there is a clear need for the use of untargeted metabolomics to quantify the metabolic activities of microbiota, this methodology has been limited by the challenge of discriminating the secondary metabolites produced by microbes from tens-of-thousands of metabolites detected in the environment. To resolve this bottleneck, we devised a computational method for recognizing and annotating putative secondary metabolites of microbial origin from fragmentation spectra. The annotations were first obtained from spectral library matching and in silico annotation ${ }^{14}$ using the GNPS web-platform ${ }^{15}$. These annotations were then queried against microbial metabolite reference databases (i.e., Natural Products Atlas ${ }^{16}$ and $\mathrm{MIBiG}^{17}$ ), and molecular networking ${ }^{18}$ was used to propagate the annotation to similar metabolites. Finally, a global chemical classification of these metabolites was achieved using a state-of-the-art annotation pipeline (i.e., SIRIUS) ${ }^{13}$. 
Shaffer et al. Metabolite-microbe profiles are shaped by the environment

We used this methodology to quantify microbial secondary metabolites from diverse microbial communities that span 20 major environments from the Earth Microbiome Project 500 (EMP500) dataset (Fig. S1, Table S1).To avoid confusion with terminology, we define 'microbial community' as consisting of members of Bacteria and Archaea. With this dataset, we show that although the presence/absence of major classes of microbially-related metabolites is indeed relatively conserved across habitats, their relative abundance reveals specific chemistry that is limited or amplified by the environment, especially when considering more resolved chemical class ontology levels (Fig. 1). Importantly, when considering differences in the relative abundances of all microbially-related metabolites, profiles among environments were so distinct that we could identify particular metabolites whose abundances were enriched in certain environments (Fig. 2a,c, Table S2, Table S3). For example, microbially-related metabolites associated with the carbohydrate pathway were especially enriched in aquatic samples, whereas those associated with the polyketide- and shikimate and phenylpropanoid pathways enriched in sediment, soil, and fungal samples (Fig. 2a). Interestingly, distinct analytical approaches identified specific metabolites as particularly important for distinguishing aquatic samples $\left(\mathrm{C}_{28} \mathrm{H}_{58} \mathrm{O}_{15}\right.$, pathway: carbohydrates, superclass: glycerolipids), non-saline plant surface samples $\left(\mathrm{C}_{13} \mathrm{H}_{10} \mathrm{O}\right.$, pathway: shikimates and phenylpropanoids, superclass: flavonoids, class: chalcones), and non-saline animal distal gut samples $\left(\mathrm{C}_{24} \mathrm{H}_{38} \mathrm{O}_{4}\right.$, pathway: terpenoids, superclass: steroids, class: cholane steroids). We also identified specific metabolites that could classify particular environments with 68.9\% accuracy in machine-learning analysis (Fig. 3a, Table S4), and found further support for the importance of particular metabolites in distinguishing environments, including the putative cholane steroid above (i.e., $\mathrm{C}_{24} \mathrm{H}_{38} \mathrm{O}_{4}$ ), and three metabolites enriched in non-saline soil and plant corpus samples (Fig. 2c, Fig. 3a, Table S4). 


\section{Shaffer et al. Metabolite-microbe profiles are shaped by the environment}

In addition to showing that the relative abundances of microbially-related metabolites distinguish environments, our results highlight the advantages of using a multi-omics approach to interpret and predict the contributions of microbes and their environments to chemical profiles in nature. Moreover, our approach illustrates that recent advances in computational annotation tools offer a powerful toolbox to interpret untargeted metabolomics data ${ }^{13}$. With these observations, we hypothesized that the differences in the relative abundances of particular metabolites among environments were due in part to underlying differences in microbial community composition and diversity. To begin to explore these relationships, we analyzed our shotgun metagenomics data and found strong correlations between microbially-related metabolite richness and microbial taxon richness for certain environments (i.e., Animal proximal gut (saline) $r=0.73, p$-value $<0.01$; Plant corpus (non-saline) $r=0.74, p$-value $<0.001$; Sediment (non-saline) $r=0.42 ., p$-value $=0.05$; Water (saline) $r=0.57, p$-value $=0.01)($ Fig. $2 b$; Table S5). We also found similarity in the clustering of samples by environment between datasets (Fig. 2c,d), and a strong correlation between sample-sample distances based on microbially related metabolites vs. microbial taxa (Table S6). Using machine-learning, we determined that specific microbial taxa and their functions could classify particular environments with $72.08 \%$ and $68.19 \%$ accuracy, respectively (Figs. S2 and S3). In addition, we examined correlations between microbe-metabolite co-occurrences learned from shotgun metagenomic profiles and (1) log-fold changes of metabolites across environments, and (2) global distributions of metabolites, and found strong relationships with each (Figure 3b). In particular, the abundances of microbially-related metabolites in aquatic samples had a strong correlation with microbe-metabolite co-occurrences (Figure 3b). In addition to highlighting such environments as 
Shaffer et al. Metabolite-microbe profiles are shaped by the environment

unique vs. other free-living and host-associated samples, this demonstrates that microbes and metabolites can be classified by- and co-occur among environments.

We further generated additional data for EMP500 samples, including gas chromatography-mass spectrometry (GC-MS) and amplicon sequence (i.e., 16S, 18S, ITS, and full-length rRNA operon) data that also supported a strong relationship between bacterial- and archaeal communities and metabolic profiles (Table S6). We anticipate that advances in genomemining will improve the discovery and classification of BGCs from MAGs and provide additional insight into these findings, and by making these data publicly available in Qiita and GNPS our data will provide an important resource for continued collaborative investigations. In the same manner, the development of novel instrumentation and computational methods for metabolomics will expand the depth of metabolites surveyed in microbiome studies.

\section{Methods. (1,000 words max)}

\section{DATASET DESCRIPTION}

Sample collection. Samples were contributed by 34 principal investigators (PIs) of the Earth Microbiome Project 500 (EMP500) Consortium. To achieve more even coverage across microbial environments, we devised an ontology of sample types (i.e., microbial environments), the EMP Ontology (EMPO) (http://www.earthmicrobiome.org/protocols-and-standards/empo//19 and selected samples to fill out EMPO categories as broadly as possible. Samples were collected following the EMP sample submission guide ${ }^{20}$. Briefly, samples were collected fresh, split into 10 aliquots, and then frozen, or alternatively collected and frozen, and subsequently split into 10 aliquots with minimal perturbation. Aliquot size was sufficient to yield 10-100 ng genomic DNA (approximately $10^{7}-10^{8}$ cells). To leave samples amenable to chemical characterization (e.g., 
Shaffer et al. Metabolite-microbe profiles are shaped by the environment

metabolomics), buffers or solutions for sample preservation (e.g., RNAlater) were avoided. Ethanol (50-95\%) was allowed as it is compatible with LC-MS/MS, although should also be avoided if possible. All sample types were stored at $-80^{\circ} \mathrm{C}$ if possible, otherwise $-20^{\circ} \mathrm{C}$. Additional details are described in the Supplementary Material.

Sample metadata. Environmental metadata was collected for all samples based on the EMP Metadata Guide ${ }^{2}$, which combines guidance from the Genomics Standards Consortium MIxS (Minimum Information about any Sequence) standard ${ }^{21}$ and the Qiita Database (https://qiita.ucsd.edu) ${ }^{22}$. The metadata guide provides templates and instructions for each MIxS environmental package (i.e., sample type). Relevant information describing each PIs submission, or study, was organized into a separate study metadata file (Table S1).

\section{METABOLOMICS}

$\boldsymbol{L} \boldsymbol{C}-\boldsymbol{M S} / \mathbf{M S}$. We used liquid chromatography with untargeted tandem mass spectrometry (LCMS/MS) to perform untargeted metabolomic profiling of all samples. Details of sample extraction and preparation, sample analysis (i.e., running the samples), data processing, data annotation, and data analysis are described in the Supplementary Material. Briefly, mass spectrometry data were normalized and converted from raw to the $m / z$ extensible markup language format (.mzML) using ProteoWizard (ver. 3.0.19, MSConvert tool) ${ }^{23}$. The mzML files were then processed with MZmine toolbox ${ }^{24}$. Data are available in the MassIVE dataset MSV000083475. The results files of MZmine were uploaded to GNPS (http://gnps.ucsd.edu) ${ }^{15}$ and analyzed with the feature-based molecular networking workflow ${ }^{18}$. 
Shaffer et al. Metabolite-microbe profiles are shaped by the environment

$\boldsymbol{G C}$-MS. We used gas chromatography to perform additional untargeted metabolomic profiling of volatile compounds from all fecal, sediment, and soil samples. Details of sample extraction and preparation, data processing and annotation, and data analysis are described in the Supplementary Material. Briefly, data were converted from raw format to the .mzML format as above, and are available in the MassIVE dataset MSV000083743. Files were uploaded to GNPS $^{15}$ and processed using the GC-MS data analysis workflow ${ }^{25}$.

\section{METAGENOMICS}

DNA extraction. For each round of extractions described below for both amplicon and shotgun metagenomic sequencing, a single aliquot of each sample was processed for DNA extraction. DNA was extracted following the EMP 96-sample, magnetic bead-based DNA extraction protocol $^{26}$, following Marotz et al. (2017) $)^{27}$, Minich et al. (2018) $)^{28}$, and Minich et al. (2019) ${ }^{29}$, and using the QIAGEN ${ }^{\circledR}$ MagAttract ${ }^{\circledR}$ PowerSoil ${ }^{\circledR}$ DNA KF Kit (384) (i.e., optimized for KingFisher). Additional details are described in the Supplementary Material.

Amplicon sequencing. We generated amplicon sequence data for variable region four (V4) of the bacterial and archaeal 16S ribosomal RNA (rRNA) gene, variable region nine (V9) of the eukaryotic 18S rRNA gene, and the fungal internal transcribed spacer one (ITS1). For amplifying and sequencing all targets, we used a low-cost, miniaturized (i.e., 5- $\mu \mathrm{L}$ volume), high-throughput (384-sample) amplicon library preparation method ${ }^{30}$. The same protocol was modified with different primer sets and PCR cycling parameters depending on the target. Two rounds of DNA extraction and sequencing were performed for each target to obtain greater coverage per sample. For a subset of 500 samples, we also generated high-quality sequence data 
Shaffer et al. Metabolite-microbe profiles are shaped by the environment

for full-length bacterial rRNA operons following the protocol described by Karst et al. $(2021)^{31}$. Additional details are described in the Supplementary Material.

Amplicon data analysis. For multi-omic analyses including amplicon sequence data, we processed each dataset for comparison of beta-diversity. Raw sequence data were converted from $\mathrm{bcl}$ to fastq, and then multiplexed files for each sequencing run uploaded as separate preparations to Qiita (study: 13114). Subsequent processing to generate feature-tables, merge data across preparations, and generate distance matrices were performed in Qiita; all other analyses were performed with QIIME2 ${ }^{32}$. Additional details are described in the Supplementary Material.

Shotgun metagenomic sequencing. One round of DNA extraction was performed for shotgun metagenomic sequencing. Sequencing libraries were prepared using a high-throughput version of the HyperPlus library chemistry (Kapa Biosystems) miniaturized to approximately 1:10 reagent volume and optimized for nanoliter-scale liquid-handling robotics ${ }^{33}$. The final pool was sequenced at Illumina on a NovaSeq6000 using S2 flow cells and 2x150-bp chemistry (Illumina, San Diego, California, USA). Additional details are described in the Supplementary Material.

Shotgun data analysis. Raw sequence data were converted from bcl to fastq, demultiplexed, and trimmed with Atropos (v1.1.24) $)^{34}$ to remove universal adapter sequences and poly-G tails introduced by the NovaSeq instrument (i.e., from use of two-color chemistry). Trimmed reads were mapped to the Web of Life database of microbial genomes ${ }^{35}$ to produce alignments that were used for both taxonomic and functional analysis of microbial communities. Additional details are described in the Supplementary Material. 
Shaffer et al. Metabolite-microbe profiles are shaped by the environment

\section{MULTI-OMICS}

Alpha-diversity correlations. Using the alpha-diversity metrics for LC-MS/MS (i.e., richness) and shotgun metagenomic taxonomic data (i.e., richness, Faith's PD, and weighted Faith's PD ${ }^{36}$ ), we performed correlation analysis to better understand their relationship. We used the function multilevel available in the R package correlation $^{37}$ to perform Spearman correlations for each environment (i.e., based on EMPO), treating study as a random effect, and adjusting for multiple comparisons using the Benjamini-Hochberg correction.

Machine-learning analyses. For machine-learning analyses of LC-MS/MS and shotgun metagenomic taxonomic- and functional data, additional samples were filtered from featuretables such to exclude environments (i.e., based on EMPO) with relatively low sample representation $(<10$ samples).

For each feature-table, we trained a Random Forest classifier for predicting environments with tuned hyperparameters with a 20-time repeated group 5-fold cross-validation approach using the $\mathrm{R}$ package caret $^{38}$. Each dataset was split into a training set (80\%) and a testing set $(20 \%)$, with similar environmental distributions in each fold iteration for the classification of samples. Additional details are described in the Supplementary Material.

Metabolite-microbe co-occurrence analysis. For co-occurrence analyses of LC-MS/MS metabolites and microbial genomes profiled from shotgun metagenomic data, feature-tables were further filtered to only retain the samples found in both datasets. We obtained co-occurrence 
Shaffer et al. Metabolite-microbe profiles are shaped by the environment

probabilities using mmvec, which we used to predict metabolites based on microbial taxa from neural-network, compositionally-robust modeling ${ }^{39}$. We then calculated the Spearman correlation between the loadings of metabolites on each PC vs. (i) betas for metabolite abundances across environments from Songbird multinomial regression, (ii) loadings for metabolites on the first three axes from the RPCA ordination ${ }^{40}$ corresponding to clustering of samples by environment, and (iii) a vector representing the global magnitude of metabolite importance across all three axes from the RPCA biplot ${ }^{40}$. We also visualized the relationships between metabolite-microbe co-occurrences and (i) by ordinating the first three PCs and coloring metabolites by their beta values (e.g., Fig. 3c). Additional details are described in the Supplementary Material.

Mantel correlations between datasets. To explore the relationships between sample-to-sample distances for any two datasets (e.g., LC-MS/MS vs. shotgun metagenomic taxonomic), we used QIIME2's diversity plugin ${ }^{32}$ to perform Mantel tests using Spearman correlations. Input distance matrices are described in the Supplementary Material. 
bioRxiv preprint doi: https://doi.org/10.1101/2021.06.04.446988; this version posted November 4, 2021. The copyright holder for this preprint (which was not certified by peer review) is the author/funder. All rights reserved. No reuse allowed without permission.

Shaffer et al. Metabolite-microbe profiles are shaped by the environment

Figures.

a

$$
\begin{array}{r}
\text { Animal proximal gut (non-saline) } \\
\text { Animal proximal gut (saline) } \\
\text { Animal distal gut (non-saline) } \\
\text { Animal distal gut (saline) } \\
\text { Animal secretion (saline) } \\
\text { Animal corpus (non-saline) } \\
\text { Animal corpus (saline) } \\
\text { Fungus corpus (non-saline) } \\
\text { Plant surface (non-saline) } \\
\text { Plant surface (saline) } \\
\text { Plant corpus (non-saline) } \\
\text { Soil (non-saline) } \\
\text { Sediment (non-saline) } \\
\text { Sediment (saline) } \\
\text { Surface (saline) } \\
\text { Subsurface (non-saline) } \\
\text { Water (non-saline) }
\end{array}
$$
Water (saline)

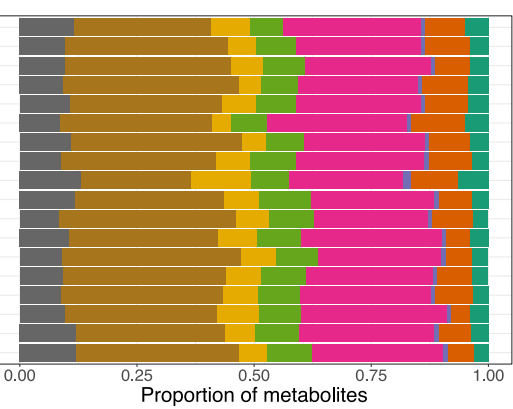

b

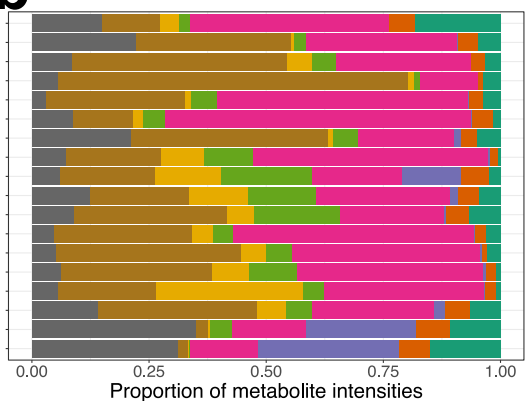

Pathway

Alkaloids
Amino acids and Peptides Carbohydrates Fatty acids
Polyketides Shikimates and Phenylpropanoids Terpenoids
Not annotated

c

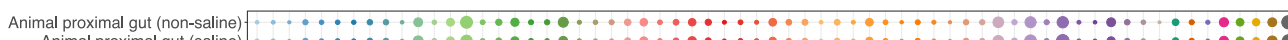

Animal proximat (saline) Animal distal gut (non-saline) Animal distal gut (saline) Animal secretion (saline)

Animal corpus (non-saline)
Animal corpus (saline)

Animal corpus (saline)
Fungus corpus (non-saline)

Plant surface (non-saline)
Plant

Plant surface (non-saline
Plant surface (saline)

Plant corpus (non-saline)

Soil (non-saline)
diment (non-saline)

Sediment (saline)

Surface (saline)
Subsurface (non-saline)

Water (non-saline

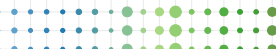

$\therefore \therefore: \cdots: \cdots \cdots:$

$\ldots \ldots \ldots \ldots \ldots \ldots$

$\therefore \ldots \ldots \cdots \cdots \cdots \cdots$

.

:

$\because \cdots \cdots$

$\therefore \therefore \cdots \cdots$

$\therefore \therefore: \cdots \cdots \cdots \cdots$

d

Animal proximal gut (non-saline)
Animal proximal gut (saline) Animal proximal gut (saline)
Animal distal gut (non-saline)

Animal distal gut (saline)
Animal

Animal secretion (saline)

Animal corpus (non-saline)

Animal corpus (saline)

ungus corpus (non-saline)
Plant surface (non-saline)

Plant surface (non-saline
Plant surface (saline

Plant surface (saline)
Plant corpus (non-saline)

Soil (non-saline)

diment (non-saline)

Sediment (saline)
Surface (saline)

Subsurface (non-saline)

Water (non-saline

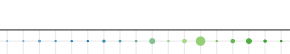

$\ldots$

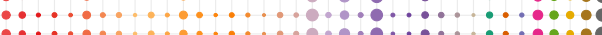

$\because \cdots \cdots \cdots \cdots \cdots \cdots \cdots \cdots \cdots \cdots \cdots \cdots \cdots$

\section{Water (saine)}

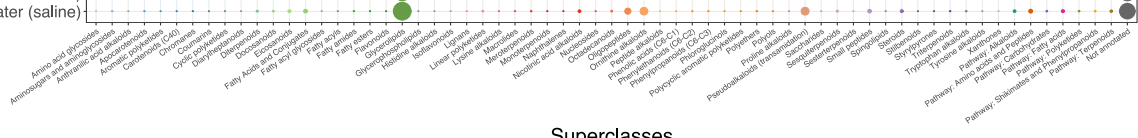

Superclasses

Fig. 1 | Distribution of microbially-related secondary metabolites chemical pathways

\section{among environments described using the Earth Microbiome Project Ontology (EMPO}

version 2, level 4). Both chemical pathway and chemical superclass annotations are shown based on presence/absence $(\mathbf{a}, \mathbf{c})$ and relative intensities $(\mathbf{b}, \mathbf{d})$ of molecular features, respectively. For superclass annotations in panels $\mathbf{c}$ and $\mathbf{d}$, we included pathway annotations for metabolites where superclass annotations were not available, when possible. 
Shaffer et al. Metabolite-microbe profiles are shaped by the environment
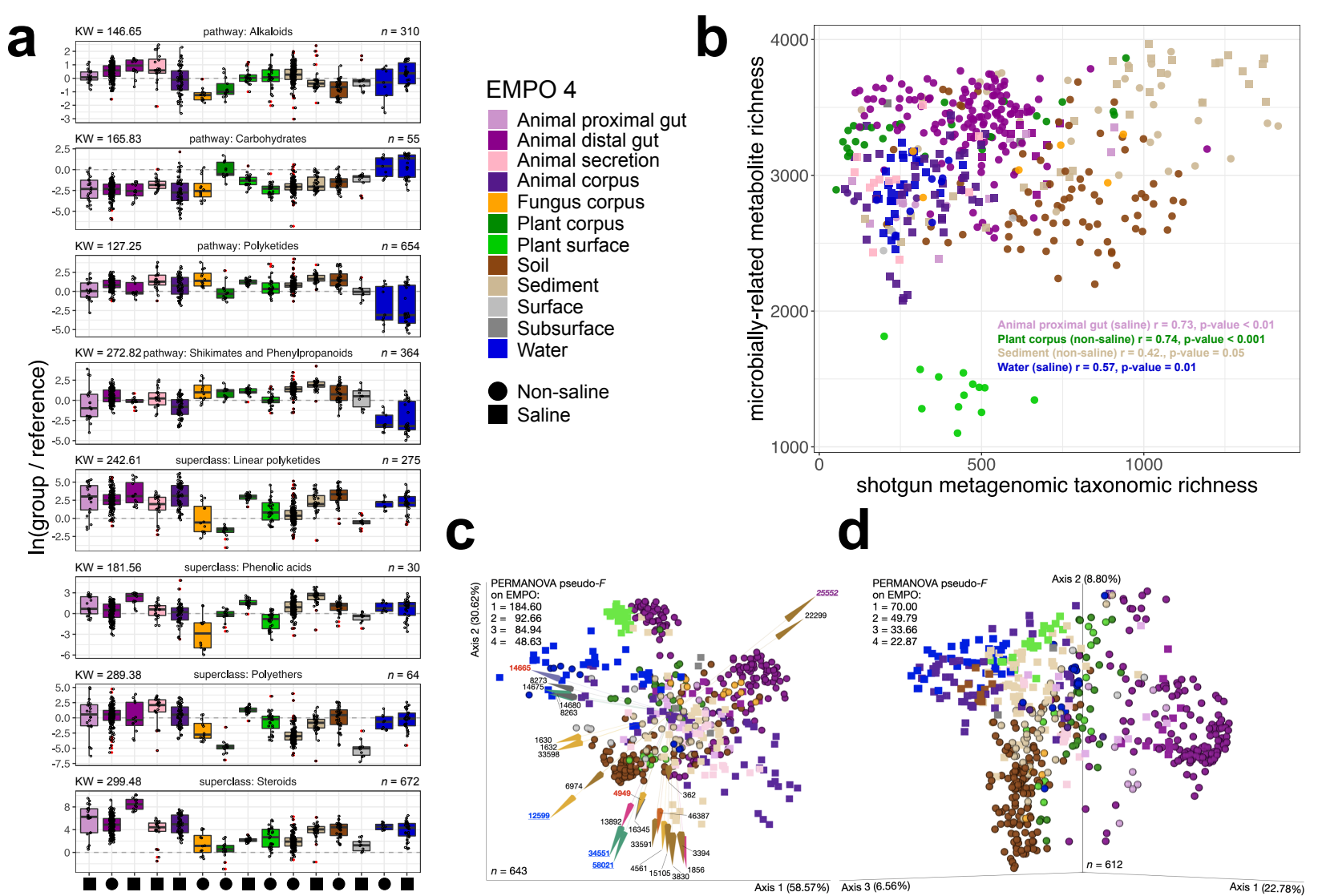

Fig. 2 | Structural-level associations between microbially-related secondary metabolites and

\section{specific environments described using the Earth Microbiome Project Ontology (EMPO}

version 2, level 4). a, Differential abundance of molecular features across environments,

highlighting four example pathways and four superclasses in separate panels. For each panel, the

$y$-axis represents the natural log-ratio of the intensities of metabolites annotated as the listed

ingroup divided by the intensities of metabolites annotated as the reference group (i.e., Amino

Acids and Peptides, $n=615$, for pathways and Flavonoids, $n=42$, for superclasses). The number

of metabolites in each ingroup is shown, as well as the chi-squared statistic from a Kruskal-

Wallis rank sum test for differences in log-ratios across environments (i.e., each test had $p$-value

$<2.2 \mathrm{E}-16)$. Each test included 606 samples. Outliers from boxplots are colored red to highlight

that they are also represented in the overlaid, jittered points. Associations between molecular

features and environments were identified using Songbird multinomial regression (model: 
Shaffer et al. Metabolite-microbe profiles are shaped by the environment

composition $=$ EMPO version 2, level 4; pseudo- $\left.Q^{2}=0.21\right)$. Additional information about features is described in Table S2. b, Relationship between microbially-related metabolite richness and microbial taxon richness across samples and environments, with significant relationships noted. Correlations with metabolite richness were weaker when using Faith's PD and weighted Faith's PD for quantifying microbial alpha-diversity (Table S5). c, Turnover in composition of microbially-related secondary metabolites across environments, visualized using Robust Aitchison Principal Components Analysis (RPCA) showing samples separated based on LC-MS/MS spectra. Shapes represent samples and are colored and shaped by EMPO. Arrows represent metabolites, and are colored by chemical pathways. The direction and magnitude of each arrow corresponds to the strength of the correlation between the relative abundance (i.e., intensity) of the metabolite it represents and the RPCA axes. Samples close to arrow heads have strong, positive associations with respective features, whereas samples at and beyond arrow origins strong, negative associations. The 25 most important metabolites are shown and are described in Table S3. Features annotated in red are those also identified in our multinomial regression analysis as among the top 10 ranked metabolites per environment (Tables S2), those in blue also separated environments in machine-learning analysis (Table S4), and those in purple identified as important in all three analyses. $\mathbf{d}$, Turnover in composition of microbial taxa across environments, visualized using Principal Coordinates Analysis (PCoA) of weighted UniFrac distances. Distances are based on counts of microbial genomes from mapping metagenomic reads to the Web of Life database. Note the similarities between panels $\mathbf{c}$ and $\mathbf{d}$ with respect to the separation of free-living (e.g., 'Water') and host-associated (e.g., 'Animal distal gut') environments along Axis 1, and a gradient from living hosts (e.g., 'Plant surface'), to detritus 
Shaffer et al. Metabolite-microbe profiles are shaped by the environment

(e.g., 'Plant corpus'), to soils and sediments along Axis 2. Results from PERMANOVA for each level of EMPO are shown (i.e., all tests had $p$-value $=0.001$ ).

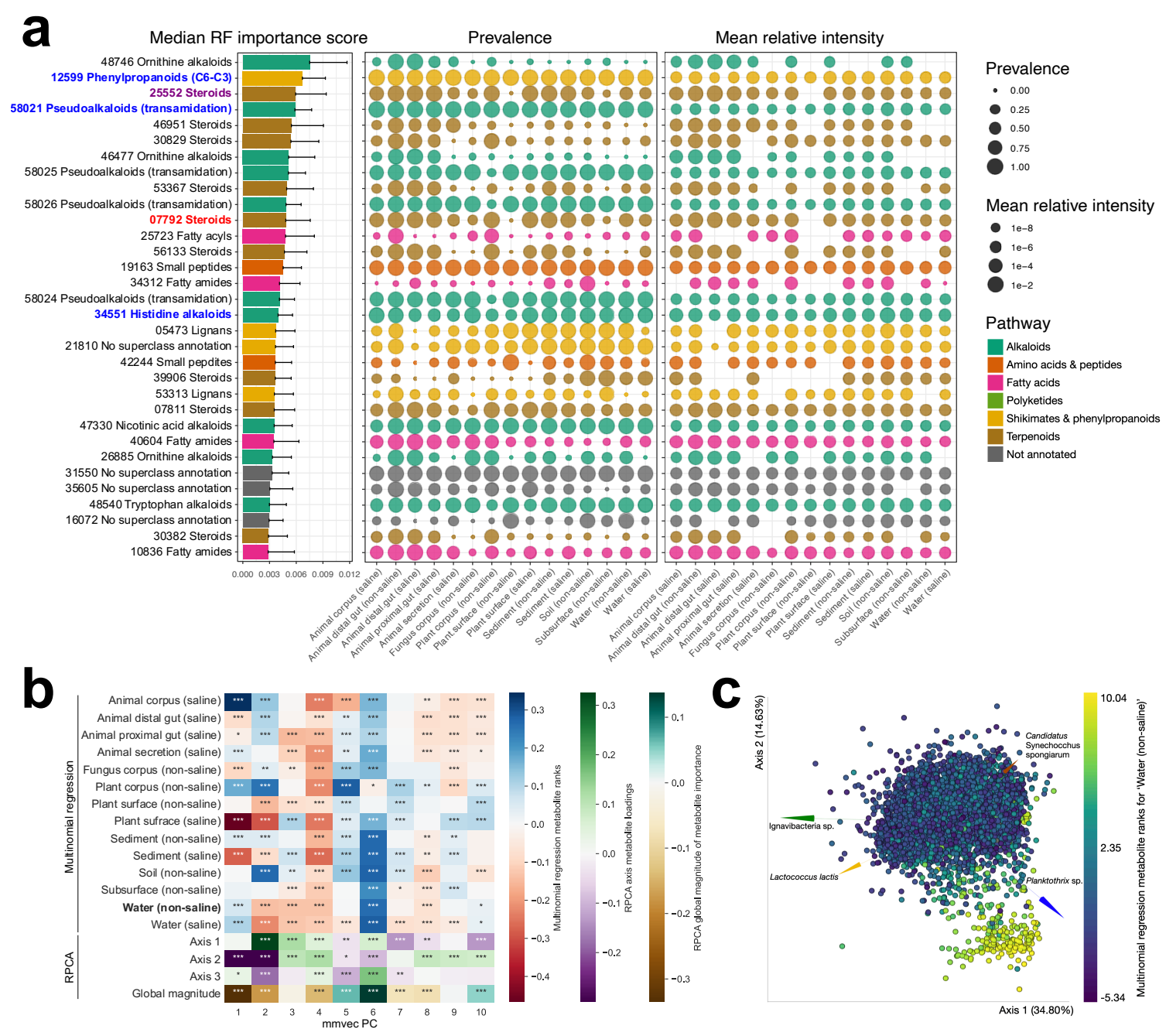

Fig. 3 | Microbially-related metabolites classify environments with a high accuracy and co-

occur with specific microbial taxa. a, The random-forest importance score, environment-wide prevalence, and mean relative intensity for the top 32 most important microbially-related metabolites contributing to the separation of environments. Metabolites are further described in Table S4. Those in red are those also identified in our multinomial regression analysis as among the top 10 ranked metabolites per environment (Tables S2), those in blue also identified to be strongly associated with RPCA axes (Fig. 2c, Table S3). b, Co-occurrence analysis results 
Shaffer et al. Metabolite-microbe profiles are shaped by the environment

showing correlation between mmvec principal coordinates (PCs) and (i) multinomial regression betas for metabolite abundances across environments, (ii) axes from the RPCA biplot in Fig. 2d corresponding to clustering of samples by environment (i.e., EMPO version 2, level 4) based on metabolite profiles, and (iii) a vector representing the global magnitude of features from the RPCA biplot in Fig. 2d. Values are Spearman correlation coefficients. Asterisks indicate significant correlations $\left({ }^{*} p<0.05,{ }^{* *} p<0.01,{ }^{* * *} p<0.001\right)$. c, The relationship between multinomial regression betas for metabolites with respect to 'Water (non-saline)' and the first three mmvec PCs shown as a multi-omics biplot of metabolite-microbe co-occurrences learned from microbial profiles. Points represent metabolites separated by their co-occurrences with microbial taxa. Metabolites are colored based on multinomial regression betas for their abundances with respect to 'Water (non-saline)'. Vectors represent specific microbial taxa strongly associated with ordination axes. The model predicting metabolite-microbe cooccurrences was more accurate than one representing a random baseline, with a pseudo- $Q^{2}$ value of 0.18 , indicating much reduced error during cross-validation. 
Shaffer et al. Metabolite-microbe profiles are shaped by the environment

\section{References.}

1. Davies, J. Specialized microbial metabolites: functions and origins. J. Antibiot. 66, 361-364 (2013).

2. Pham, J. V. et al. A Review of the Microbial Production of Bioactive Natural Products and Biologics. Front. Microbiol. 10, 1404 (2019).

3. Nayfach, S. et al. A genomic catalog of Earth's microbiomes. Nat. Biotechnol. (2020) doi:10.1038/s41587-020-0718-6.

4. Ziemert, N., Alanjary, M. \& Weber, T. The evolution of genome mining in microbes - a review. Nat. Prod. Rep. 33, 988-1005 (2016).

5. Libis, V. et al. Uncovering the biosynthetic potential of rare metagenomic DNA using cooccurrence network analysis of targeted sequences. Nat. Commun. 10, 3848 (2019).

6. Hugerth, L. W. et al. Metagenome-assembled genomes uncover a global brackish microbiome. Genome Biol. 16, 279 (2015).

7. Tully, B. J., Graham, E. D. \& Heidelberg, J. F. The reconstruction of 2,631 draft metagenome-assembled genomes from the global oceans. Sci Data 5, 170203 (2018).

8. Stewart, R. D. et al. Compendium of 4,941 rumen metagenome-assembled genomes for rumen microbiome biology and enzyme discovery. Nat. Biotechnol. 37, 953-961 (2019).

9. Van Goethem, M. W. et al. Long-read metagenomics of soil communities reveals phylumspecific secondary metabolite dynamics. Cold Spring Harbor Laboratory 2021.01.23.426502 (2021) doi:10.1101/2021.01.23.426502.

10. Amos, G. C. A. et al. Comparative transcriptomics as a guide to natural product discovery and biosynthetic gene cluster functionality. Proc. Natl. Acad. Sci. U. S. A. 114, E11121E11130 (2017). 
Shaffer et al. Metabolite-microbe profiles are shaped by the environment

11. Vogel, C. \& Marcotte, E. M. Insights into the regulation of protein abundance from proteomic and transcriptomic analyses. Nat. Rev. Genet. 13, 227-232 (2012).

12. Aksenov, A. A., da Silva, R., Knight, R., Lopes, N. P. \& Dorrestein, P. C. Global chemical analysis of biology by mass spectrometry. Nature Reviews Chemistry 1, 0054 (2017).

13. Dührkop, K. et al. Systematic classification of unknown metabolites using high-resolution fragmentation mass spectra. Nat. Biotechnol. (2020) doi:10.1038/s41587-020-0740-8.

14. Mohimani, H. et al. Dereplication of microbial metabolites through database search of mass spectra. Nat. Commun. 9, 4035 (2018).

15. Wang, M. et al. Sharing and community curation of mass spectrometry data with Global Natural Products Social Molecular Networking. Nat. Biotechnol. 34, 828-837 (2016).

16. van Santen, J. A. et al. The Natural Products Atlas: An Open Access Knowledge Base for Microbial Natural Products Discovery. ACS Cent Sci 5, 1824-1833 (2019).

17. Kautsar, S. A. et al. MIBiG 2.0: a repository for biosynthetic gene clusters of known function. Nucleic Acids Res. 48, D454-D458 (2020).

18. Nothias, L.-F. et al. Feature-based molecular networking in the GNPS analysis environment. Nat. Methods 17, 905-908 (2020).

19. Thompson, L. R. et al. A communal catalogue reveals Earth's multiscale microbial diversity. Nature 551, 457-463 (2017).

20. Thompson, L. et al. EMP Sample Submission Guide v1 (protocols.io.pfqdjmw). protocols.io (2018) doi:10.17504/protocols.io.pfqdjmw.

21. Yilmaz, P. et al. Minimum information about a marker gene sequence (MIMARKS) and minimum information about any (x) sequence (MIxS) specifications. Nat. Biotechnol. 29, 415-420 (2011). 
Shaffer et al. Metabolite-microbe profiles are shaped by the environment

22. Gonzalez, A. et al. Qiita: rapid, web-enabled microbiome meta-analysis. Nat. Methods 15, 796-798 (2018).

23. Chambers, M. C. et al. A cross-platform toolkit for mass spectrometry and proteomics. Nat. Biotechnol. 30, 918-920 (2012).

24. Pluskal, T., Castillo, S., Villar-Briones, A. \& Oresic, M. MZmine 2: modular framework for processing, visualizing, and analyzing mass spectrometry-based molecular profile data. $B M C$ Bioinformatics 11, 395 (2010).

25. Aksenov, A. A. et al. Auto-deconvolution and molecular networking of gas chromatographymass spectrometry data. Nat. Biotechnol. 39, 169-173 (2021).

26. Marotz, L. et al. Earth Microbiome Project (EMP) high throughput (HTP) DNA extraction protocol v1 (protocols.io.pdmdi46). protocols.io (2018) doi:10.17504/protocols.io.pdmdi46.

27. Marotz, C. et al. DNA extraction for streamlined metagenomics of diverse environmental samples. Biotechniques 62, 290-293 (2017).

28. Minich, J. J. et al. KatharoSeq Enables High-Throughput Microbiome Analysis from LowBiomass Samples. mSystems 3, (2018).

29. Minich, J. J. et al. Quantifying and Understanding Well-to-Well Contamination in Microbiome Research. mSystems 4, (2019).

30. Minich, J. J. et al. High-Throughput Miniaturized 16S rRNA Amplicon Library Preparation Reduces Costs while Preserving Microbiome Integrity. mSystems 3, (2018).

31. Karst, S. M. et al. High-accuracy long-read amplicon sequences using unique molecular identifiers with Nanopore or PacBio sequencing. Nat. Methods 18, 165-169 (2021). 
Shaffer et al. Metabolite-microbe profiles are shaped by the environment

32. Bolyen, E. et al. Reproducible, interactive, scalable and extensible microbiome data science using QIIME 2. Nat. Biotechnol. 37, 852-857 (2019).

33. Sanders, J. G. et al. Optimizing sequencing protocols for leaderboard metagenomics by combining long and short reads. Genome Biol. 20, 226 (2019).

34. Didion, J. P., Martin, M. \& Collins, F. S. Atropos: specific, sensitive, and speedy trimming of sequencing reads. PeerJ 5, e3720 (2017).

35. Zhu, Q. et al. Phylogenomics of 10,575 genomes reveals evolutionary proximity between domains Bacteria and Archaea. Nat. Commun. 10, 5477 (2019).

36. Swenson, N. G. Functional and Phylogenetic Ecology in R. (Springer, New York, NY, 2014). doi:10.1007/978-1-4614-9542-0.

37. Makowski, D., et al. Methods and Algorithms for Correlation Analysis in R. Journal of Open Source Software, 5, 2306 (2019).

38. Kuhn, M. Classification and Regression Training [R package caret version 6.0-86]. (2020).

39. Morton, J. T. et al. Learning representations of microbe-metabolite interactions. Nat. Methods 16, 1306-1314 (2019).

40. Martino, C. et al. A Novel Sparse Compositional Technique Reveals Microbial Perturbations. mSystems 4, (2019). 
Shaffer et al. Metabolite-microbe profiles are shaped by the environment

\section{Acknowledgements.}

We thank Lindsay Goldasich and Julia Toronczak for assistance with sample processing for sequencing; Marcus Fedarko, Rachel Diner, Joshua Ladau, Elisha Wood-Charlson, Stephen

Nayfach, Daniel Udwary, and Emiley Eloe-Fadrosh for reviewing the manuscript. This work was supported in part by the United States (US) National Institute of Health (NIH) (awards 1RF1AG058942-01, 1DP1AT010885, R01HL140976, R01DK102932, R01HL134887,

U19AG063744, U01 AI124316), US Department of Agriculture - National Institute of Food and Agriculture (USDA-NIFA) (award 2019-67013-29137), the US National Science Foundation (NSF) - Center for Aerosol Impacts on Chemistry of the Environment, Crohn's \& Colitis Foundation Award (CCFA) (award 675191), US Department of Energy - Office of Science Office of Biological and Environmental Research - Environmental System Science Program, Semiconductor Research Corporation and Defense Advanced Research Projects Agency (SRC/DARPA) (award GI18518), Department of Defense (award W81XWH-17-1-0589), the Office of Naval Research (ONR) (award N00014-15-1-2809), the Emerald Foundation (award 3022), IBM Research AI through the AI Horizons Network, and the Center for Microbiome Innovation. J.P.S. was supported by NIH/NIGMS IRACDA K12 GM068524. L.F.N. was supported by the NIH (award R01-GM107550). A.D.B. was supported by the Danish Council for Independent Research (DFF) (award 9058-00025B). W.B. was supported by the Research Foundation - Flanders (12W0418N). K.D. and S.B. were supported by Deutsche Forschungsgemeinschaft (BO 1910/20 and 1910/23). P.C.D. was supported by the Gordon and Betty Moore Foundation (award GBMF7622) and the NIH (award R01-GM107550).

Metabolomics analyses at Pacific Northwest National Laboratory (PNNL) were supported by the Laboratory Directed Research and Development program via the Microbiomes in Transition 
Shaffer et al. Metabolite-microbe profiles are shaped by the environment

Initiative and performed in the Environmental Molecular Sciences Laboratory, a national scientific user facility sponsored by the US Office of Biological and Environmental Research and located at PNNL. This contribution originates in part from the River Corridor Scientific Focus Area project at PNNL. PNNL is a multiprogram national laboratory operated by Battelle for the Department of Energy (DOE) under contract DE-AC05-76RLO 1830. We thank Eppendorf, Illumina, and Integrated DNA Technologies for in-kind support at various phases of the project.

\section{Author contributions.}

J.A.G., J.K.J., and R.K. conceived the idea for the project. P.C.D., and R.K. designed the multiomics component of the project and provided project oversight. J.P.S. managed the project, performed preliminary data exploration, coordinated data analysis, analyzed data, and provided data interpretation. L.F.N. coordinated and performed LC-MS/MS sample processing, coordinated and performed annotation of LC-MS/MS, analyzed LC-MS/MS data, and provided LC-MS/MS data interpretation. L.R.T. designed the multi-omics component of the project, solicited sample collection, curated sample metadata, processed samples, performed preliminary data exploration, and provided project oversight. J.G.S. designed the multi-omics component, managed the project, developed protocols and tools, coordinated and performed sequencing, and performed preliminary exploration of sequence data. R.A.S. developed protocols and coordinated and performed sequencing. S.P.C. and T.O.M. coordinated and performed GC-MS sample processing and provided interpretation of GC-MS data. A.D.B. conceived the idea for the paper, performed preliminary data exploration, analyzed data, and provided data interpretation. S.H. performed machine-learning analyses. F.L. performed co-occurrence analysis, multinomial 
Shaffer et al. Metabolite-microbe profiles are shaped by the environment

regression analyses, and correlations with co-occurrence data. H.L.L. performed multinomial regression analyses. Q.Z. developed tools and provided interpretation of shotgun metagenomics data. C.Mart. and J.T.M. provided oversight and interpretation of RPCA, multinomial regression, and co-occurrence analyses. S.K. performed preliminary exploration of shotgun metagenomics data. K.D., S.B, and H.W.K. annotated LC-MS/MS data. A.A.A. processed GC-MS data. W.B. provided oversight for machine-learning analyses. C.Maro. processed samples for sequencing. Y.V.B. performed preliminary data exploration and provided oversight for machine-learning analysis. A.T. and D.P. performed preliminary data exploration. L.P., A.P.C., N.H., and K.L.B. performed preliminary exploration of shotgun metagenomic data and performed machine learning analyses. P.D. performed preliminary exploration of shotgun metagenomics data. A.G. developed tools, provided interpretation of shotgun metagenomics data, and analyzed shotgun metagenomics data. G.H. coordinated short-read amplicon and shotgun metagenomics sequencing. M.M.B. and K.S. performed short-read amplicon and shotgun metagenomics sequencing. T.S. assisted with DNA extraction. D.M. coordinated long-read amplicon sequencing, analyzed shotgun metagenomics data, and provided interpretation of the data. S.M.K. and M.A. coordinated and performed long-read amplicon sequencing and long-read sequence data analysis. J.J.M. collected samples, coordinated field logistics, developed protocols, and performed short-read amplicon and shotgun metagenomics sequencing. S.S. collected samples, coordinated field logistics, and provided interpretation of the data. G.L.A. curated sample metadata and organized sequence data. J.D. processed sequence data. A.D.S. provided project oversight and data interpretation. T.T., A.S., and J.S. collected samples, coordinated field logistics, and provided interpretation of the data. J.P.S. and L.F.N. wrote the manuscript, with contributions from all authors. 
Shaffer et al. Metabolite-microbe profiles are shaped by the environment

\section{Earth Microbiome Project 500 (EMP500) Consortium.}

Jennifer L. Bowen ${ }^{1}$, Max Chavarría ${ }^{2,3}$, Don A. Cowan ${ }^{4}$, Jaime Huerta-Cepas ${ }^{5}$, Paul Jensen ${ }^{6}$, Lingjing Jiang ${ }^{7}$, Anton Lavrinienko ${ }^{8}$, Thulani P. Makhalanyane ${ }^{4}$, Tapio Mappes ${ }^{8}$, Ezequiel M. Marzinelli $^{9}$, Sou Miyake ${ }^{10}$, Timothy A. Mousseau ${ }^{8}$, Catalina Murillo-Cruz $^{2}$, Adrián A. PintoTomás $^{2}$, Dorota L. Porazinska ${ }^{11}$, Jean-Baptiste Ramond ${ }^{4,12}$, Taniya RoyChowdhury ${ }^{13,14}$, Henning Seedorf $^{10,13}$, J. Reuben Shipway ${ }^{16,17}$, Frank J. Stewart ${ }^{18}$, Jana M. U'Ren ${ }^{19}$, Phillip C. Watts ${ }^{8}$, Nicole S. Webster ${ }^{20,21}$, Jesse R. Zaneveld ${ }^{22}$, Shan Zhang ${ }^{23}$

${ }^{1}$ Northeastern University, Boston, Massachusetts, USA. ${ }^{2}$ University of Costa Rica, San José, Costa Rica. ${ }^{3}$ CENIBiot, San José, Costa Rica. ${ }^{4}$ University of Pretoria, Pretoria, South Africa. ${ }^{5}$ Universidad Politécnica de Madrid, Instituto Nacional de Investigación y Tecnología Agraria y Alimentaria, Madrid, Spain. ${ }^{6}$ University of California San Diego, La Jolla, California, USA.

${ }^{7}$ Janssen Research \& Development, San Diego, California, USA. ${ }^{8}$ University of Jyväskylä, Jyväskylä, Finland. ${ }^{9}$ The University of Sydney, Sydney, Australia. ${ }^{10}$ Temasek Life Sciences Laboratory, Singapore, Singapore. ${ }^{11}$ University of Florida, Gainesville, Florida, USA.

${ }^{12}$ Pontificia Universidad Católica de Chile, Santiago, Chile. ${ }^{13}$ Pacific Northwest National Laboratory, Richland, Washington, USA. ${ }^{14}$ University of Maryland, College Park, Maryland, USA. ${ }^{15}$ National University of Singapore, Singapore, Singapore. ${ }^{16}$ University of Portsmouth, Portsmouth, United Kingdom. ${ }^{17}$ University of Massachusetts Amherst, Amherst, Massachusetts, USA. ${ }^{18}$ Montana State University, Bozeman, Montana, USA. ${ }^{19}$ University of Arizona, Tucson, Arizona, USA. ${ }^{20}$ Australian Institute of Marine Science, Townsville, Qld, Australia. ${ }^{21}$ University 
Shaffer et al. Metabolite-microbe profiles are shaped by the environment

of Queensland, Brisbane, Australia. ${ }^{22}$ University of Washington Bothell, Bothell, Washington, USA. ${ }^{23}$ University of New South Wales, Sydney, Australia.

\section{Competing interests.}

S.B. and K.D. are co-founders of Bright Giant GmbH. 
Shaffer et al. Metabolite-microbe profiles are shaped by the environment

\section{Supplementary Material.}

\section{SUPPLEMENTARY METHODS}

\section{DATASET DESCRIPTION}

Sample collection. Samples were contributed by 34 principal investigators of the Earth Microbiome Project 500 (EMP500) Consortium. To achieve more even coverage across microbial environments, we devised an ontology of sample types (microbial environments), the EMP Ontology (EMPO) (http://www.earthmicrobiome.org/protocols-and-standards/empo/) ${ }^{1}$ and selected samples to fill out EMPO categories as broadly as possible. Samples were collected following the Earth Microbiome Project sample submission guide 2 . Briefly, samples were collected fresh, split into 10 aliquots, and then frozen, or alternatively collected and frozen, and subsequently split into 10 aliquots with minimal perturbation. Aliquot size was sufficient to yield 10-100 ng genomic DNA (approximately $10^{7}-10^{8}$ cells). To leave samples amenable to chemical characterization (metabolomics), buffers or solutions for sample preservation (e.g., RNAlater) were avoided. Ethanol (50-95\%) was allowed as it is compatible with LC-MS/MS though should also be avoided if possible.

Sampling guidance was tailored for four general sample types: bulk unaltered (e.g., soil, sediment, feces), bulk fractionated (e.g., sponges, corals, turbid water), swabs (e.g., biofilms), and filters. Bulk unaltered samples were split fresh (or frozen) sampled into 10 pre-labeled 2-mL screw-cap bead beater tubes (Sarstedt cat. no. 72.694 .005 or similar), ideally with at least $200 \mathrm{mg}$ biomass, and flash frozen in liquid nitrogen (if possible). Bulk fractionated samples were fractionated as appropriate for the sample type, split into 10 pre-labeled $2-\mathrm{mL}$ screw-cap bead 
Shaffer et al. Metabolite-microbe profiles are shaped by the environment

beater tubes, ideally with at least $200 \mathrm{mg}$ biomass, and flash frozen in liquid nitrogen (if possible). Swabs were collected as 10 replicate swabs using 5 BD SWUBE dual cotton swabs with wooden stick and screw cap (cat. no. 281130). Filters were collected as 10 replicate filters (47 mm diameter, 0.2 um pore size, polyethersulfone (preferred) or hydrophilic PTFE filters), placed in a pre-labeled 2-mL screw-cap bead beater tubes, and flash frozen in liquid nitrogen (if possible). All sample types were stored at $-80{ }^{\circ} \mathrm{C}$ if possible, otherwise $-20{ }^{\circ} \mathrm{C}$.

To track the provenance of sample aliquots, we employed a QR coding scheme. Labels were affixed to aliquot tubes before shipping, when possible. QR codes had the format "name.99.s003.a05", where "name" is the PI name, "99" is the study ID, "s003" is the sample number, and "a05" is the aliquot number. QR codes (version 2, 25 pixels x 25 pixels) were printed on 1.125" x 0.75" rectangular and 0.437" circular cap Cryogenic Direct Thermal Labels (GA International, part no. DFP-70) using a Zebra model GK420d printer and ZebraDesigner Pro software for Windows. After receipt but before aliquots were stored in freezers, QR codes were scanned into a sample inventory spreadsheet using a QR scanner.

Sample metadata. Environmental metadata was collected for all samples based on the EMP Metadata Guide ${ }^{2}$, which combines guidance from the Genomics Standards Consortium MIxS (Minimum Information about any Sequence) standard ${ }^{3}$ and the Qiita Database (https://qiita.ucsd.edu) ${ }^{4}$. The metadata guide provides templates and instructions for each MIxS environmental package (i.e., sample type). Relevant information describing each PI submission, or study, was organized into a separate study metadata file (Table S1).

\section{METABOLOMICS}


Shaffer et al. Metabolite-microbe profiles are shaped by the environment

LC-MS/MS sample extraction and preparation. All solvents and reactants used were LC-MS grade. To maximize the biomass extracted from each sample, the samples were prepared depending on their sampling method (e.g. bulk, swabs, filter, and controls). The bulk samples were transferred into a microcentrifuge tube (polypropylene, PP) and dissolved in 7:3 $\mathrm{MeOH}: \mathrm{H}_{2} \mathrm{O}$ using a volume varying from $600 \mu \mathrm{L}$ to $1.5 \mathrm{~mL}$, depending on the amounts of sample available, and homogenized in a tissue-lyser (QIAGEN) at $25 \mathrm{~Hz}$ for $5 \mathrm{~min}$. Then, the tubes were centrifuged at 15,000 rpm for $15 \mathrm{~min}$, and the supernatant was collected in a 96-well plate (PP). For swabs, the swabs were transferred into a 96-well plate (PP) and dissolved in 1.0 $\mathrm{mL}$ of 9:1 EtOH: $\mathrm{H}_{2} \mathrm{O}$. The prepared plates were sonicated for $30 \mathrm{~min}$, and after 12 hours at $4^{\circ} \mathrm{C}$, the swabs were removed from the wells. The filter samples were dissolved in $1.5 \mathrm{~mL}$ of $7: 3$ $\mathrm{MeOH}: \mathrm{H}_{2} \mathrm{O}$ in microcentrifuge tubes (PP) and were sonicated for $30 \mathrm{~min}$. After 12 hours at $4^{\circ} \mathrm{C}$, the filters were removed from the tubes. The tubes were centrifuged at 15,000 rpm for $15 \mathrm{~min}$, and the supernatants were transferred to $96-$ well plates (PP). The process control samples (bags, filters, and tubes) were prepared by adding $3.0 \mathrm{~mL}$ of $2: 8 \mathrm{MeOH}: \mathrm{H}_{2} \mathrm{O}$ and by recovering $1.5 \mathrm{~mL}$ after two minutes. After the extraction process, all sample plates were dried with a vacuum concentrator and subjected to solid phase extraction (SPE). The SPE is used to remove salts that could reduce ionization efficiency during mass spectrometry analysis, as well as the most polar and non-polar compounds (e.g., waxes) that cannot be analyzed efficiently by reversed-phase chromatography. The protocol was as follows: the samples (in plates) were dissolved in $300 \mu \mathrm{L}$ of 7:3 MeOH: $\mathrm{H}_{2} \mathrm{O}$ and put in an ultrasound bath for $20 \mathrm{~min}$. SPE was performed with SPE plates (Oasis HLB, Hydrophilic-Lipophilic-Balance, $30 \mathrm{mg}$ with particle sizes of $30 \mu \mathrm{m}$ ). The SPE beds were activated by priming them with $100 \% \mathrm{MeOH}$, and equilibrated with $100 \% \mathrm{H}_{2} \mathrm{O}$. The samples were loaded on the SPE beds, and $600 \mu \mathrm{L} \mathrm{100 \%} \mathrm{H}_{2} \mathrm{O}$ was used as wash solvent. The 
Shaffer et al. Metabolite-microbe profiles are shaped by the environment

eluted washing solution was discarded, as it contains salts and very polar metabolites that subsequent metabolomics analysis is not designed to handle. The sample elution was carried out sequentially with $600 \mu \mathrm{L}$ 7:3 MeOH: $\mathrm{H}_{2} \mathrm{O}$ and with $600 \mu \mathrm{L} 100 \% \mathrm{MeOH}$. The obtained plates were dried with a vacuum concentrator. For mass spectrometry analysis, the samples were resuspended in $130 \mu \mathrm{L}$ of 7:3 MeOH: $\mathrm{H}_{2} \mathrm{O}$ containing $0.2 \mu \mathrm{M}$ of amitriptyline as an internal standard. The plates were centrifuged at $2,000 \mathrm{rpm}$ for $15 \mathrm{~min}$ at $4^{\circ} \mathrm{C} .100 \mu \mathrm{L}$ of samples were transferred into a new 96-well plate (PP) for mass spectrometry analysis.

LC-MS/MS sample analysis. The extracted samples were analyzed by ultra-high performance liquid chromatography (UHPLC, Vanquish, Thermo Scientific, Waltham, Massachusetts, USA) coupled to a quadrupole-Orbitrap mass spectrometer (Q Exactive, Thermo Scientific, Waltham, Massachusetts, USA) operated in data-dependent acquisition mode (LC-MS/MS in DDA mode). Chromatographic separation was performed using a Kinetex $\mathrm{C}_{18}$ 1.7- $\mathrm{mm}$ (Phenomenex, Torrance, California, USA), 100-Å pore size, 2.1-mm (internal diameter) x 50-mm (length) column with a $\mathrm{C}_{18}$ guard cartridge (Phenomenex). The column was maintained at $40^{\circ} \mathrm{C}$. The mobile phase was composed of a mixture of (A) water with $0.1 \%$ formic acid $(\mathrm{v} / \mathrm{v})$ and (B) acetonitrile with $0.1 \%$ formic acid. Chromatographic elution method was set as follows: 0.00 $1.00 \mathrm{~min}$, isocratic $5 \% \mathrm{~B} ; 1.00-9.00 \mathrm{~min}$, gradient from $5 \%$ to $100 \% \mathrm{~B} ; 9.00-11.00 \mathrm{~min}$, isocratic $100 \% \mathrm{~B}$; and followed by equilibration $11.00-11.50 \mathrm{~min}$, gradient from $100 \%$ to $5 \% \mathrm{~B}$; $11.50-12.50 \mathrm{~min}$, isocratic $5 \% \mathrm{~B}$. The flow rate was set to $0.5 \mathrm{~mL} / \mathrm{min}$.

The UHPLC was interfaced to the orbitrap using a heated electrospray ionization (HESI) source with the following parameters: ionization mode: positive, spray voltage, $+3496.2 \mathrm{~V}$; heater temperature, $363.90^{\circ} \mathrm{C}$; capillary temperature, $377.50^{\circ} \mathrm{C}$; S-lens RF, 60 (arb. units); sheath gas 
Shaffer et al. Metabolite-microbe profiles are shaped by the environment

flow rate, 60.19 (arb. units); and auxiliary gas flow rate, 20.00 (arb. units). The $\mathrm{MS}^{1}$ scans were acquired at a resolution (at $\mathrm{m} / \mathrm{z} 200$ ) of 35,000 in the $\mathrm{m} / \mathrm{z} 100-1500$ range, and the fragmentation spectra $\left(\mathrm{MS}^{2}\right)$ scans at a resolution of 17,500 from 0.0 to $12.5 \mathrm{~min}$. The automatic gain control (AGC) target and maximum injection time were set at $1 \times 10^{6}$ and $160 \mathrm{~ms}$ for $\mathrm{MS}^{1}$ scans, and set at $5 \times 10^{5}$ and $220 \mathrm{~ms}$ for $\mathrm{MS}^{2}$ scans, respectively. Up to three $\mathrm{MS}^{2}$ scans in data-dependent mode (Top 3) were acquired for the most abundant ions per $\mathrm{MS}^{1}$ scans using the apex trigger mode (4 to $15 \mathrm{~s}$ ), dynamic exclusion ( $11 \mathrm{~s}$ ), and automatic isotope exclusion. The starting value for $\mathrm{MS}^{2}$ was $m / z$ 50. Higher-energy collision induced dissociation (HCD) was performed with a normalized collision energy of 20,30, $40 \mathrm{eV}$ in stepped mode. The major background ions originating from the SPE were excluded manually from the $\mathrm{MS}^{2}$ acquisition. Analyses were randomized within a plate and blank samples analyzed every 20 injections. A QC mix sample assembled from 20 random samples across the sample-types was injected at the beginning, the middle, and the end of each plate sequence. The chromatographic shift observed throughout the batch is estimated as less than $2 \mathrm{~s}$, and the relative standard deviation of ion intensity was $15 \%$ per replicates.

LC-MS/MS data processing. The mass spectrometry data were centroided and converted from the proprietary format (.raw) to the $m / z$ extensible markup language format (.mzML) using ProteoWizard (ver. 3.0.19, MSConvert tool) ${ }^{\frac{5}{}}$. The mzML files were then processed with MZmine toolbox $\underline{6}$ using the ion-identity networking modules $\underline{7}$ that allows advanced detection for adduct/isotopologue annotations. The MZmine processing was performed on Ubuntu 18.04 LTS 64-bits workstation (Intel Xeon E5-2637, 3.5 GHz, 8 cores, 64 Gb of RAM) and took ca. 3 d. The MZmine project, the MZmine batch file (.XML format), and results files (.MGF and .CSV) 
Shaffer et al. Metabolite-microbe profiles are shaped by the environment

are available in the MassIVE dataset MSV000083475. The MZmine batch file contains all the parameters used during the processing. Briefly, feature detection and deconvolution was

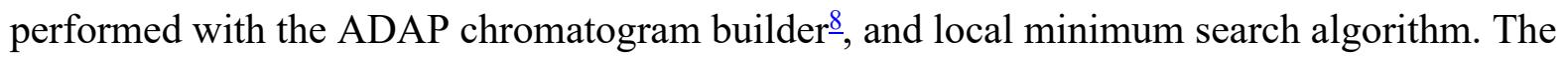
isotopologues were regrouped, and the features (i.e., peaks) were aligned across samples. The aligned peak list was gap filled and only peaks with an associated fragmentation spectrum and occurring in a minimum of 3 files were conserved. Peak shape correlation analysis grouped peaks originating from the same molecule, and to annotate adduct/isotopologue with ion-identity networking ${ }^{2}$. Finally the feature quantification table results (.CSV) and spectral information (.MGF) were exported with the GNPS module for feature-based molecular networking analysis on GNPS ${ }^{9}$ and with SIRIUS export modules.

LC-MS/MS data annotation. The results files of MZmine (.MGF and .CSV files) were uploaded to GNPS (http://gnps.ucsd.edu $)^{10}$ and analyzed with the feature-based molecular networking workflow ${ }^{9}$. Spectral library matching was performed against public fragmentation spectra $\left(\mathrm{MS}^{2}\right)$ spectral libraries on GNPS and the NIST17 library.

For the additional annotation of small peptides, the DEREPLICATOR tools were used via GNPS $\frac{11,12}{\text {. The MS }}{ }^{2}$ spectra were systematically annotated with the SIRIUS computational tools $\frac{13}{}$ computational annotation tool (v. 4.4.25, headless, Linux). Molecular formulas were computed with the SIRIUS module by matching the experimental and predicted isotopic patterns $\frac{14}{4}$, and from fragmentation trees analysis $\frac{15}{2}$ of $\mathrm{MS}^{2}$. Molecular formula prediction was refined with the ZODIAC module using Gibbs sampling $\underline{16}$ on the fragmentation spectra (chimeric spectra or had a poor fragmentation were excluded). 
Shaffer et al. Metabolite-microbe profiles are shaped by the environment

In silico structure annotation using structures from biodatabase was done with CSI:FingerID ${ }^{17}$. Systematic class annotations were obtained with CANOPUS $\underline{18}$ and used the NPClassifier ontology $\frac{19}{}$. The parameters for SIRIUS tools were set as follows, for SIRIUS: molecular formula candidates retained (80), molecular formula database (ALL), maximum precursor ion $\mathrm{m} / \mathrm{z}$ computed (750), profile (orbitrap), $\mathrm{m} / \mathrm{z}$ maximum deviation (10 ppm), ions annotated with MZmine were prioritized and other ions were considered $([\mathrm{M}+\mathrm{H} 3 \mathrm{~N}+\mathrm{H}]+,[\mathrm{M}+\mathrm{H}]+$, $[\mathrm{M}+\mathrm{K}]+,[\mathrm{M}+\mathrm{Na}]+,[\mathrm{M}+\mathrm{H}-\mathrm{H} 2 \mathrm{O}]+,[\mathrm{M}+\mathrm{H}-\mathrm{H} 4 \mathrm{O} 2]+,[\mathrm{M}+\mathrm{NH} 4]+)$; for ZODIAC: the features were split into 10 random subsets for lower computational burden and computed separately with the following parameters: threshold filter (0.9), minimum local connections (0); for CSI:FingerID: $m / z$ maximum deviation (10 ppm) and biological database (BIO).

To establish putative microbially-related secondary metabolites, we collected annotations from spectral library matching and the DEREPLICATOR tools and queried them against the largest microbial metabolite reference databases (Natural Products Atlas $\underline{20}$ and $\mathrm{MIBiG}^{21}$ ). Molecular networking 9 was then used to propagate the annotation of microbially-related secondary metabolites to throughout all molecular families (i.e., the network component).

LC-MS/MS data analysis. We combined the annotation results from the different tools described above to create a comprehensive feature metadata file. Using that information we generated a feature-table including only secondary metabolite features determined to be microbially-related. We then excluded very low-intensity features introduced to certain samples during the gap-filing step described above. These features were identified based on presence in negative controls that were universal to all sample types (i.e., bulk, filter, and swab), and by their relatively low persample intensity values. Finally, we excluded features present in positive controls for sampling 
Shaffer et al. Metabolite-microbe profiles are shaped by the environment

devices specific to each sample type (i.e., bulk, filter, or swab). The final feature-table contained 618 samples and 6,588 putative microbially-related secondary metabolite features that were used for subsequent analysis.

We used QIIME 2's 22 diversity plugin to quantify alpha-diversity (i.e., feature richness) for each sample, and deicode to quantify beta-diversity (i.e., robust Aitchison distance) $)^{23}$ between each pair of samples. We parameterized our robust Aitchison Principal Components Analysis $(\mathrm{RPCA})^{23}$ to exclude samples with fewer than 500 features, and features present in fewer than $10 \%$ of samples. We used the taxa plugin to quantify the relative abundance of microbiallyrelated secondary metabolite pathways and superclasses (i.e., based on NPClassifier) within each environment (i.e., for each level of EMPO, version 2 level 4), and songbird ${ }^{24}$ to identify sets of microbially-related secondary metabolites whose abundances were associated with certain environments. We parameterized our songbird model as follows: epochs $=1,000,000$, differential prior $=0.5$, learning rate $=1 \mathrm{E}-05$, summary interval $=2$, batch size $=400$, minimum sample count $=0$, and training on $80 \%$ of samples at each level of EMPO version 2, level 4 using 'Animal proximal gut (non-saline)' as the reference environment.

We used the RPCA biplot and EMPeror ${ }^{25}$ to visualize differences in composition among samples, as well as the association with samples of the 25 most influential microbially-related secondary metabolite features (i.e., those with the largest magnitude across the first three principal component loadings). We tested for significant differences in metabolite composition across the various levels of EMPO (version 2) using permutational multivariate analysis of

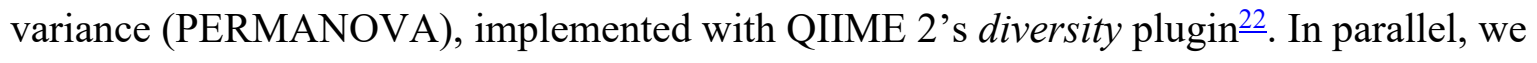
visualized significant differences in the relative abundances of specific microbially-related secondary metabolite pathways and superclasses within each environment by first selecting 
Shaffer et al. Metabolite-microbe profiles are shaped by the environment

features and calculating log-ratios using qurro ${ }^{26}$, and then plotting using the ggplot 2 package $\underline{27}$ in $\mathrm{R} \underline{28}$. We tested for significant differences in relative abundances across environments using Kruskal-Wallis tests, implemented using the base stats package in $\mathrm{R} \underline{28}$.

GC-MS sample extraction and preparation. Two protocols were used for sample extraction, one for the 105 soil samples and another for the 356 fecal and sediment samples which were treated as biosafety level 2. The 105 soil samples were received at the Pacific Northwest National Laboratory and processed as follows. Each soil sample (1 g) was weighed into microcentrifuge tubes (Biopur Safe-Lock, $2.0 \mathrm{~mL}$, Eppendorf, Hamburg, Germany). $1 \mathrm{~mL}$ of $\mathrm{H}_{2} \mathrm{O}$ and one scoop $(\sim 0.5 \mathrm{~g})$ of a $1: 1(\mathrm{v} / \mathrm{v})$ mixture of garnet $(0.15 \mathrm{~mm}$, Omni International, Kennesaw, Georgia, USA) and stainless steel (SS) (0.9 - 2.0 mm blend, Next Advance, Troy, New York) beads and one $3 \mathrm{~mm}$ SS bead (Qiagen, Hilden, Germany) was added to each tube. Samples were homogenized in a tissue lyser (Qiagen, Hilden, Germany) for $3 \mathrm{~min}$ at $30 \mathrm{~Hz}$ and transferred into $15 \mathrm{~mL}$ polypropylene tubes (Olympus, Genesee Scientific, San Diego, California, USA). Icecold water $(1 \mathrm{~mL})$ was used to rinse the smaller tube and combined into the $15 \mathrm{~mL}$ tube. $10 \mathrm{~mL}$ of $2: 1(\mathrm{v} / \mathrm{v})$ chloroform:methanol added and samples were rotated at $4^{\circ} \mathrm{C}$ for $10 \mathrm{~min}$ followed by cooling at $-70^{\circ} \mathrm{C}$ for $10 \mathrm{~min}$ and centrifuged at $4,000 \mathrm{rpm}$ for $10 \mathrm{~min}$ to separate phases. The top and bottom layers were combined into $40 \mathrm{~mL}$ glass vials and dried using a vacuum concentrator. $1 \mathrm{~mL}$ of 2:1 chloroform:methanol was added to each large glass vial and the sample was transferred into $1.5 \mathrm{~mL}$ tubes and centrifuged at $12,000 \mathrm{~g}$. The supernatant was transferred into glass vials and dried for derivatization.

The remaining 356 samples that were received from UCSD that included fecal and sediment samples were processed as follows: $100 \mu \mathrm{L}$ of each sample was transferred to a $2 \mathrm{~mL}$ 
Shaffer et al. Metabolite-microbe profiles are shaped by the environment

microcentrifuge tube using a scoop (MSP01, Next Advance, Tustin, California, USA). The final volume of sample was brought to $1.5 \mathrm{~mL}$ ensuring the solvent ratio is $3: 8: 4 \mathrm{H}_{2} \mathrm{O}: \mathrm{CHCl}_{3}: \mathrm{MeOH}$ by adding the appropriate volumes of $\mathrm{H}_{2} \mathrm{O}, \mathrm{MeOH}$, and $\mathrm{CHCl}_{3}$. After transfer, one 3-mm SS bead (QIAGEN), $400 \mu \mathrm{L}$ of methanol, and $300 \mu \mathrm{L}$ of $\mathrm{H} 2 \mathrm{O}$ were added to each tube and the samples were vortexed for $30 \mathrm{~s}$. Then, $800 \mu \mathrm{L}$ of chloroform was added and samples were vortexed for $30 \mathrm{~s}$. After centrifuging at 4,000 rpm for $10 \mathrm{~min}$ to separate phases, the top and bottom layers were combined in a vial and dried for derivatization.

The samples were derivatized for GC-MS analysis as follows: $20 \mu \mathrm{L}$ of a methoxyamine solution in pyridine $(30 \mathrm{mg} / \mathrm{mL})$ was added to the sample vial and vortexed for $30 \mathrm{~s}$. A bath sonicator was used to ensure the sample was completely dissolved. Samples were incubated at $37^{\circ} \mathrm{C}$ for $1.5 \mathrm{~h}$ while shaking at 1,000 rpm. $80 \mu \mathrm{L}$ of $\mathrm{N}$-methyl-N-trimethylsilyltrifluoroacetamide and $1 \%$ trimethylchlorosilane (MSTFA) solution was added and samples were vortexed for $10 \mathrm{~s}$, followed by incubation at $37^{\circ} \mathrm{C}$ for $30 \mathrm{~min}$ with $1000 \mathrm{rpm}$ shaking. The samples were then transferred into a vial with an insert.

An Agilent 7890A gas chromatograph coupled with a single quadrupole 5975C mass spectrometer (Agilent Technologies, Santa Clara, California, USA) and a HP-5MS column (30 m $\times 0.25 \mathrm{~mm} \times 0.25 \mu \mathrm{m}$; Agilent Technologies, Santa Clara, California, USA) was used for untargeted analysis. Samples $(1 \mu \mathrm{L})$ were injected in splitless mode, and the helium gas flow rate was determined by the Agilent Retention Time Locking function based on analysis of deuterated myristic acid (Agilent Technologies, Santa Clara, California, USA). The injection port temperature was held at $250^{\circ} \mathrm{C}$ throughout the analysis. The $\mathrm{GC}$ oven was held at $60^{\circ} \mathrm{C}$ for $1 \mathrm{~min}$ after injection, and the temperature was then increased to $325^{\circ} \mathrm{C}$ by $10^{\circ} \mathrm{C} / \mathrm{min}$, followed by a 10 min hold at $325^{\circ} \mathrm{C}$. Data were collected over the mass range of $m / z 50-600$. A mixture of 
Shaffer et al. Metabolite-microbe profiles are shaped by the environment

FAMEs (C8-C28) was analyzed each day with the samples for retention index alignment purposes during subsequent data analysis.

GC-MS data processing and annotation. The data were converted from vendor's format to the .mzML format and processed using GNPS GC-MS data analysis workflow (https://gnps.ucsd.edu) ${ }^{\underline{29}}$. The compounds were identified by matching experimental spectra to the public libraries available at GNPS as well as NIST 17 and Wiley libraries. The data are publicly available at the MassIVE depository (https://massive.ucsd.edu); dataset ID:

MSV000083743. The GNPS deconvolution can be found at the following link: https://gnps.ucsd.edu/ProteoSAFe/status.jsp?task=d5c5135a59eb48779216615e8d5cb3ac The library search can be found at the following link: https://gnps.ucsd.edu/ProteoSAFe/status.jsp?task=59b20fc8381 f4ee6b79d35034de81d86

GC-MS data analysis. For multi-omic analyses including GC-MS data, we first removed noisy features by excluding those with GNPS balance scores $<50$. We then estimated beta-diversity for each dataset using robust Aitchison distances ${ }^{23}$. The final feature table for GC-MS contained 460 samples and 216 features that were used for subsequent analysis.

\section{METAGENOMICS}

DNA extraction. For each round of extractions described below for both amplicon and shotgun metagenomic sequencing, a single aliquot of each sample was processed for DNA extraction. DNA was extracted following the EMP 96-sample, magnetic bead-based DNA extraction protocol $\frac{30}{}$, following Marotz et al. (2017) $)^{\frac{31}{1}}$, Minich et al. (2018) $)^{\frac{32}{2}}$, and Minich et al. (2019) 
Shaffer et al. Metabolite-microbe profiles are shaped by the environment

and using the QIAGEN® MagAttract ${ }^{\circledR}$ PowerSoil ${ }^{\circledR}$ DNA KF Kit (384) (i.e., optimized for KingFisher). Importantly, material from each sample was added to a unique bead tube (containing garnet beads) for single-tube lysis, which has been shown to reduce sample-to-

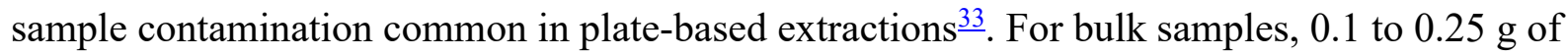
material was added to each well; for filtered samples, one entire filter was added to each well; for swabbed samples, one swab head was added to each well. The lysis solution was dissolved at $60^{\circ} \mathrm{C}$ before addition to each tube, then capped tubes were incubated at $65^{\circ} \mathrm{C}$ for 10 min prior to mechanical lysis at $6000 \mathrm{rpm}$ for 20 min using a MagNA Lyser (Roche Diagnostics, California, USA). Lysate from each bead tube was then randomly assigned and added to wells of a 96-well plate, and then cleaned-up using the KingFisher Flex system (Thermo Scientific, Waltham, Massachusetts, USA), and resulting DNA stored at $-20^{\circ} \mathrm{C}$ for sequencing. We note that whereas QIAGEN does not offer a 'hybrid' extraction kit allowing for single-tube lysis and plate-based clean-up, the Thermo MagMAX Microbiome Ultra kit does, and was recently shown to be comparable to the EMP protocol used here $\underline{34}$.

Amplicon sequencing. We generated amplicon sequence data for variable region four (V4) of the bacterial and archaeal 16S ribosomal RNA (rRNA) gene, variable region nine (V9) of the eukaryotic 18S rRNA gene, and the fungal internal transcribed spacer one (ITS1). For amplifying and sequencing all targets, we used a low-cost, miniaturized (i.e., 5- $\mu \mathrm{L}$ volume), high-throughput (384-sample) amplicon library preparation method implementing the Echo 550

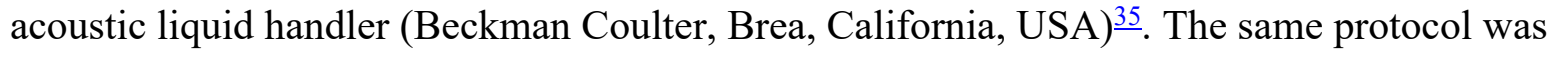
modified with different primer sets and PCR cycling parameters depending on the target. Two rounds of DNA extraction and sequencing were performed for each target to obtain greater 
Shaffer et al. Metabolite-microbe profiles are shaped by the environment

coverage per sample. For a subset of 500 samples, we also generated high-quality sequence data for full-length bacterial rRNA operons following the protocol described by Karst et al. (2021) which is outlined briefly below.

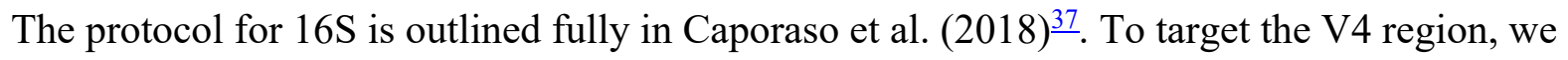
used the primers 515F (Parada) (5'-GTGYCAGCMGCCGCGGTAA-3') and 806R (Apprill) (5'GGACTACNVGGGTWTCTAAT-3'). These primers are updated from the original EMP 16S-

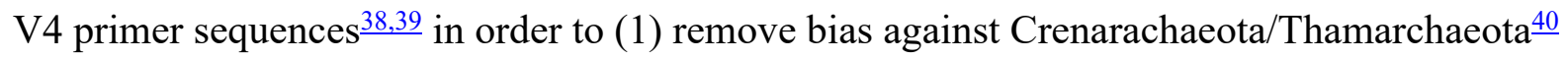
and the marine freshwater clade SAR11 (Alphaproteobacteria) ${ }^{41}$, and (2) enable the use of various reverse primer constructs (e.g., the V4-V5 region using the reverse primer $926 \mathrm{R} \underline{42}$ by moving the barcode/index to the forward primer ${ }^{40}$ ). We note that whereas we previously named these updated primers " $515 \mathrm{FB}$ " and " $806 \mathrm{RB}$ " to distinguish them from the original primers, the "B" may be misinterpreted to indicate "Barcode". To avoid ambiguity, we now use the original names suffixed with the lead author name (i.e., "515F (Parada)", "806R (Apprill)", and "926R (Quince)". We highly recommend to always check the primer sequence in addition to the primer name. For Qiita users, studies with "library_construction_protocol” as "515f/806rbc" used the original primers, whereas " $515 \mathrm{fbc} / 806 \mathrm{r}$ " indicates use of updated primers, where "bc" refers to the location of barcode.

To facilitate sequencing on Illumina platforms, the following primer constructs were used to integrate adapter sequences during amplification $\underline{38,39,43}$

(https://msystems.asm.org/content/1/1/e00009-15). For the barcoded forward primer, constructs included (5' to 3'): the 5' Illumina adapter

(AATGATACGGCGACCACCGAGATCTACACGCT), a Golay barcode (variable), a forward primer pad (TATGGTAATT), a forward primer linker (GT), and the forward primer (515F 
Shaffer et al. Metabolite-microbe profiles are shaped by the environment

[Parada]) (GTGYCAGCMGCCGCGGTAA). For the reverse primer, constructs included (5' to 3'): The reverse complement of 3' Illumina adapter (CAAGCAGAAGACGGCATACGAGAT), a reverse primer pad (AGTCAGCCAG), a reverse primer linker (CC), and the reverse primer (806R [Apprill]) (GGACTACNVGGGTWTCTAAT).

For each $25-\mu \mathrm{L}$ reaction, we combined $13 \mu \mathrm{L}$ PCR-grade water (Sigma St. Louis, MO, USA, cat. no. W3500 or QIAGEN, Hilden, Germany, cat. no. 17000-10), $10 \mu$ L Platinum Hot Start PCR Master Mix (2X) (Thermo Scientific, Waltham, Massachusetts, USA, cat. no. 13000014), $0.5 \mu \mathrm{L}$ of each primer $(10 \mu \mathrm{M})$, and $1 \mu \mathrm{L}$ of template DNA. The final concentration of the master mix in each $1 \mathrm{X}$ reaction was $0.8 \mathrm{X}$ and that of each primer was $0.2 \mu \mathrm{M}$. Cycling parameters for a 384well thermal cycler were as follows: $94^{\circ} \mathrm{C}$ for $3 \mathrm{~min}$; 35 cycles of $94^{\circ} \mathrm{C}$ for $1 \mathrm{~min}, 50^{\circ} \mathrm{C}$ for 1 $\min$, and $72^{\circ} \mathrm{C}$ for $105 \mathrm{~s}$; and $72^{\circ} \mathrm{C}$ for $10 \mathrm{~min}$. For a 96 -well thermal cycler, we recommend the following: $94^{\circ} \mathrm{C}$ for $3 \mathrm{~min}$; 35 cycles of $94^{\circ} \mathrm{C}$ for $45 \mathrm{~s}, 50^{\circ} \mathrm{C}$ for $1 \mathrm{~min}$, and $72^{\circ} \mathrm{C}$ for $90 \mathrm{~s}$; and $72^{\circ} \mathrm{C}$ for $10 \mathrm{~min}$.

We amplified each sample in triplicate (i.e., each sample was amplified in three replicate $25-\mu \mathrm{L}$ reactions), and pooled products from replicate reactions for each sample into a single volume (75 $\mu \mathrm{L})$. We visualized expected products between $300-350 \mathrm{bp}$ on agarose gels, and note that whereas low-biomass samples may yield no visible bands, instruments such as a Bioanalyzer or TapeStation (Agilent, Santa Clara, California, USA) can be used to confirm amplification. We quantified amplicons using the Quant-iT PicoGreen dsDNA Assay Kit (Thermo Scientific, Waltham, MA, USA, cat. no. P11496), following the manufacturer's instructions. To pool samples, we combined an equal amount of product from each sample (240 ng) into a single tube, and cleaned the pool using the UltraClean PCR Clean-Up Kit (QIAGEN, Hilden, Germany, cat. no. 12596-4), following the manufacturer's instructions. We checked DNA quality using a 
Shaffer et al. Metabolite-microbe profiles are shaped by the environment

Nanodrop (Thermo Scientific, Waltham, Massachusetts, USA), confirming A260/A280 ratios were between 1.8-2.0.

For sequencing, the following primer constructs were used. Read 1 constructs included (5' to 3'): a forward primer pad (TATGGTAATT), a forward primer linker (GT), and the forward primer (515F [Parada]) (GTGYCAGCMGCCGCGGTAA). Read 2 constructs included (5' to 3'): a reverse primer pad (AGTCAGCCAG), a reverse primer linker (CC), and the reverse primer (806R [Apprill]) (GGACTACNVGGGTWTCTAAT). The index primer sequence was AATGATACGGCGACCACCGAGATCTACACGCT, which we highlight as having an extra GCT at the 3' end compared to Illumina's index primer sequence, in order to increase the $\mathrm{T}_{\mathrm{m}}$ for read 1 during sequencing.

The protocol for $18 \mathrm{~S}$ is outlined fully in Amaral-Zettler et al. (2018) $\underline{44}$. To target variable region nine (V9), we used the primers $1391 \mathrm{f}$ (5'-GTACACACCGCCCGTC-3') and EukBr (5'TGATCCTTCTGCAGGTTCACCTAC-3'). These primers are based on those of Amaral-Zettler et al. (2009) $)^{45}$ and Stoek et al. (2010) ${ }^{46}$, and are designed for use with Illumina platforms. The forward primer is a universal small-subunit primer, whereas the reverse primer favors eukaryotes but with mismatches can bind and amplify Bacteria and Archaea. In addition to deviations from the $16 \mathrm{~S}$ protocol above with respect to primer construct sequences and PCR cycling parameters, we included a blocking primer that reduces amplification of vertebrate host DNA for host-

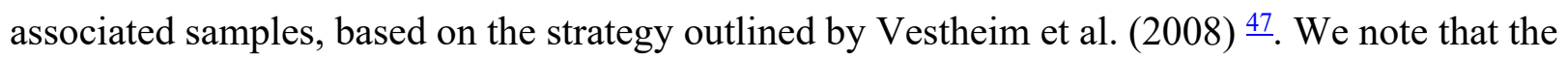
blocking primer is particularly useful for host-associated samples with a low biomass of eukaryotic DNA.

The following primer constructs were used to integrate adapter sequences during amplification. For the barcoded forward primer, constructs included ( $5^{\prime}$ to 3 '): the 5' Illumina adapter 
Shaffer et al. Metabolite-microbe profiles are shaped by the environment

(AATGATACGGCGACCACCGAGATCTACAC), a forward primer pad (TATCGCCGTT), a forward primer linker (CG), and the forward primer(Illumina_Euk_1391f)

(GTACACACCGCCCGTC). For the reverse primer, constructs included (5' to $3^{\prime}$ ): The reverse complement of 3' Illumina adapter (CAAGCAGAAGACGGCATACGAGAT), a Golay barcode (variable), a reverse primer pad (AGTCAGTCAG), a reverse primer linker (CA), and the reverse primer (806R [Apprill]) (TGATCCTTCTGCAGGTTCACCTAC). The construct for the blocking primer is as such and is formatted for ordering from IDT (Coralville, Iowa, USA): “GCCCGTCGCTACTACCGATTGG/ideoxyI//ideoxyI//ideoxyI//ideoxyI//ideoxyI/TTAGTGAG GCCCT/3SpC3/”.

Reaction mixtures without the blocking primer were prepared as described for $16 \mathrm{~S}$. For reactions including the blocking primer, we combined $9 \mu \mathrm{L}$ PCR-grade water, $10 \mu \mathrm{L}$ master mix, $0.5 \mu \mathrm{L}$ of each primer $(10 \mu \mathrm{M}), 4 \mu \mathrm{L}$ of blocking primer $(10 \mu \mathrm{M})$, and $1 \mu \mathrm{L}$ of template DNA. The final concentration of the master mix in each $1 \mathrm{X}$ reaction was $0.8 \mathrm{X}$, that of each primer was $0.2 \mu \mathrm{M}$, and that of the blocking primer was $1.6 \mu \mathrm{M}$. Without blocking primers, cycling parameters for a 384-well thermal cycler were as follows: $94^{\circ} \mathrm{C}$ for $3 \mathrm{~min} ; 35$ cycles of $94^{\circ} \mathrm{C}$ for $45 \mathrm{~s}, 57^{\circ} \mathrm{C}$ for 1 min, and $72^{\circ} \mathrm{C}$ for $90 \mathrm{~s}$; and $72^{\circ} \mathrm{C}$ for $10 \mathrm{~min}$. With blocking primers, cycling parameters for a 384-well thermal cycler were as follows: $94^{\circ} \mathrm{C}$ for $3 \mathrm{~min}$; 35 cycles of $94^{\circ} \mathrm{C}$ for $45 \mathrm{~s}, 65^{\circ} \mathrm{C}$ for $15 \mathrm{~s}, 57^{\circ} \mathrm{C}$ for $30 \mathrm{~s}$, and $72^{\circ} \mathrm{C}$ for $90 \mathrm{~s}$; and $72^{\circ} \mathrm{C}$ for $10 \mathrm{~min}$. Expected bands ranged between 210-310 bp.

For sequencing, the following primer constructs were used. Read 1 constructs

(Euk_illumina_read1_seq_primer) included ( $5^{\prime}$ to $3^{\prime}$ ): a forward primer pad (TATCGCCGTT), a forward primer linker (CG), and the forward primer (1391f) (GTACACACCGCCCGTC). Read 2 constructs (Euk_illumina_read2_seq_primer) included ( $5^{\prime}$ to $3^{\prime}$ ): a reverse primer pad 
Shaffer et al. Metabolite-microbe profiles are shaped by the environment

(AGTCAGTCAG), a reverse primer linker (CA), and the reverse primer (EukBr)

(TGATCCTTCTGCAGGTTCACCTAC). The index primer construct

(Euk_illumina_index_seq_primer) included (5' to 3'): the reverse complement of the reverse primer (EukBr) (GTAGGTGAACCTGCAGAAGGATCA), the reverse complement of the reverse primer linker (TG), and the reverse complement of the reverse primer pad (CTGACTGACT).

The protocol for ITS is outlined fully in Smith et al. (2018) $\underline{48}$. To target the fungal internal transcribed spacer (ITS1), we used the primers ITS1f (5'-CTTGGTCATTTAGAGGAAGTAA3') and ITS2 (5'-GCTGCGTTCTTCATCGATGC-3'). These primers are based on those of

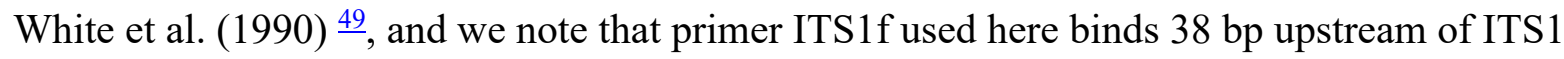
reported in that study.

The following primer constructs were used to integrate adapter sequences during amplification. For the barcoded forward primer, constructs included ( $5^{\prime}$ to $3^{\prime}$ ): the 5' Illumina adapter (AATGATACGGCGACCACCGAGATCTACAC), a forward primer linker (GG), and the forward primer (ITS1f) (CTTGGTCATTTAGAGGAAGTAA). For the reverse primer, constructs included (5' to 3'): The reverse complement of 3' Illumina adapter (CAAGCAGAAGACGGCATACGAGAT), a Golay barcode (variable), a reverse primer linker (CG), and the reverse primer (ITS2) (GCTGCGTTCTTCATCGATGC).

Reaction mixtures without the blocking primer were prepared as described for 16S. Cycling parameters for a 384 -well thermal cycler were as follows: $94^{\circ} \mathrm{C}$ for $1 \mathrm{~min} ; 35$ cycles of $94^{\circ} \mathrm{C}$ for $30 \mathrm{~s}, 52^{\circ} \mathrm{C}$ for $30 \mathrm{~s}$, and $68^{\circ} \mathrm{C}$ for $30 \mathrm{~s}$; and $68^{\circ} \mathrm{C}$ for $10 \mathrm{~min}$. Expected bands ranged between $250-600 \mathrm{bp}^{\underline{50,51} .}$ 
Shaffer et al. Metabolite-microbe profiles are shaped by the environment

For sequencing, the following primer constructs were used. Read 1 sequencing primer constructs included (5' to 3'): a forward primer segment (TTGGTCATTTAGAGGAAGTAA), and a region extending into the amplicon (AAGTCGTAACAAGGTTTCC). Read 2 sequencing primer constructs included ( $5^{\prime}$ to $3^{\prime}$ ): a reverse primer segment (CGTTCTTCATCGATGC), and a region extending into the amplicon (VAGARCCAAGAGATC). The index sequencing primer construct included $\left(5^{\prime}\right.$ to $\left.3^{\prime}\right)$ : the reverse complement of the region extending into the amplicon (TCTC), the reverse complement of the reverse primer (GCATCGATGAAGAACGCAGC), and the reverse complement of the linker $(\mathrm{CG})$.

The protocol for generating bacterial full-length rRNA operon data is described in Karst et al. $(2021)^{36}$. The method uses a unique molecular identifier (UMI) strategy to remove PCR errors and chimeras resulting in a mean error rate of $0.0007 \%$ and a chimera rate of $0.02 \%$ of the final amplicon data. Briefly, the bacterial rRNA operons were targeted with an initial PCR using tailed versions of 27f ( AGRGTTYGATYMTGGCTCAG) ${ }^{52}$ and 2490r

(GACGGGCGGTGWGTRCA) $^{53}$. The primer tails contained synthetic priming sites and $18 \mathrm{bp}$ long patterned UMIs (NNNYRNNNYRNNNYRNNN). The PCR reaction (50 $\mu \mathrm{L})$ contained 1-2 ng DNA template, 1U Platinum SuperFi DNA Polymerase High Fidelity (Thermo Fisher Scientific, Waltham, Massachusetts, USA) and a final concentration of $1 \times$ SuperFi buffer, $0.2 \mathrm{mM}$ of each dNTP, $500 \mathrm{nM}$ of each tailed $27 \mathrm{f}$ and tailed $2490 \mathrm{r}$. The PCR program consisted of initial denaturation $\left(3 \mathrm{~min}\right.$ at $\left.95^{\circ} \mathrm{C}\right)$ and two cycles of denaturation $\left(30 \mathrm{~s}\right.$ at $\left.95^{\circ} \mathrm{C}\right)$, annealing $(30$ $\mathrm{s}$ at $\left.55^{\circ} \mathrm{C}\right)$ and extension $\left(6 \mathrm{~min}\right.$ at $\left.72^{\circ} \mathrm{C}\right)$. The PCR product was purified using a custom bead purification protocol 'SPRI size selection protocol for $>1.5-2 \mathrm{~kb}$ DNA fragments' (ONT)'. The resulting product is uniquely tagged rRNA operon amplicons. The uniquely tagged rRNA operons were amplified in a second PCR, where the reaction $(100 \mu \mathrm{L})$ contained $2 \mathrm{U}$ Platinum 
Shaffer et al. Metabolite-microbe profiles are shaped by the environment

SuperFi DNA Polymerase High Fidelity (Thermo Fisher Scientific, Waltham, Massachusetts, USA) and a final concentration of $1 \mathrm{X}$ SuperFi buffer, $0.2 \mathrm{mM}$ of each dNTP, $500 \mathrm{nM}$ of each forward and reverse synthetic primer targeting the tailed primers from above. The PCR program consisted of initial denaturation $\left(3 \mathrm{~min}\right.$ at $\left.95^{\circ} \mathrm{C}\right)$ and then $25-35$ cycles of denaturation $(15 \mathrm{~s}$ at $\left.95^{\circ} \mathrm{C}\right)$, annealing $\left(30 \mathrm{~s}\right.$ at $\left.60^{\circ} \mathrm{C}\right)$ and extension $\left(6 \mathrm{~min}\right.$ at $\left.72^{\circ} \mathrm{C}\right)$ followed by final extension $(5$ min at $72^{\circ} \mathrm{C}$ ). The PCR product was purified using a custom bead purification protocol. Batches of 25 amplicon libraries were barcoded and sent for PacBio Sequel II library preparation and sequencing (Sequel II SMRT Cell 8M and $30 \mathrm{~h}$ collection time) at the DNA Sequencing Center at Brigham Young University. CCS reads were generated using CCS v.3.4.1

(https://github.com/PacificBiosciences/ccs) using default settings. UMI consensus sequences were generated using the longread_umi pipeline (https://github.com/SorenKarst/longread_umi) using the following command: longread_umi pacbio_pipeline -d ccs_reads.fq -o out_dir -m 3500 -M 6000 -s 60 -e 60 -f CAAGCAGAAGACGGCATACGAGAT -F

\section{AGRGTTYGATYMTGGCTCAG -r AATGATACGGCGACCACCGAGATC -R} CGACATCGAGGTGCCAAAC -U '0.75;1.5;2;0' -c 2.

Amplicon data analysis. For multi-omic analyses including amplicon sequence data, we processed each dataset for comparison of beta-diversity. Raw sequence data were converted from bcl to fastq, and then multiplexed files for each sequencing run uploaded as separate preparations to Qiita (study: 13114). For each sequencing run, data were then demultiplexed, trimmed to 150bp, and denoised using Deblur ${ }^{54}$ to generate a feature-table of sub-operational taxonomic units (sOTUs) per sample. 
Shaffer et al. Metabolite-microbe profiles are shaped by the environment

For each $16 \mathrm{~S}$ sequencing run, we placed denoised reads into the GreenGenes $13 \_8$ phylogeny $^{55}$ via fragment insertion using SATé-Enabled Phylogenetic Placement (SEPP) ${ }^{56}$, to produce a phylogeny for diversity analyses. Reads (i.e., sOTUs) not placed during SEPP were removed from each feature-table. We then merged feature-tables across sequencing runs, excluded singleton sOTUs, rarefied to 5,000 features per sample, and estimated beta-diversity using unweighted UniFrac distances, using QIIME2's ${ }^{22}$ diversity plugin. The final feature-table for $16 \mathrm{~S}$ beta-diversity analysis had 681 samples and 93,260 features.

For $18 \mathrm{~S}$ data, we first merged feature-tables across sequencing runs, and classified taxonomy for each sOTU via pre-fitted machine-learning classifiers ${ }^{57}$, using the SILVA 138 reference database ${ }^{58}$. We then excluded singleton sOTUs and samples with a total frequency $<3,000$ features, and estimated beta-diversity for each dataset using robust Aitchison distances ${ }^{23}$. The final feature table for $18 \mathrm{~S}$ beta-diversity analysis had 496 samples and 40,587 features.

For fungal ITS data separately, we first merged feature-tables across sequencing runs, and classified taxonomy for each sOTU as above but using UNITE 8 reference database ${ }^{59}$. We then excluded singleton sOTUs and samples with a total frequency $<500$ features, and estimated beta-diversity for each dataset using robust Aitchison distances ${ }^{23}$. The final feature table for fungal ITS beta-diversity analysis had 488 samples and 10,821 features.

For full-length rRNA operon data, per-sample fasta files were re-formatted for importing to QIIME2 as SampleData[Sequences] (i.e., with each header as $'>\{\text { sample_identifier }\}_{-}\{$sequence_identifier $\left.\}\right)$, concatenated into a single fasta file, and imported. We then used QIIME2's vsearch plugin ${ }^{60}$ to dereplicate sequences and then cluster them at $65 \%$ similarity (i.e., due to rapid evolution at bacterial ITS regions). The $65 \%$ OTU feature-table had 365 samples and 285 features. The concatenated fasta file and $65 \%$ OTU 
Shaffer et al. Metabolite-microbe profiles are shaped by the environment

feature-table were uploaded to Qiita as a distinct preparations (study: 13114). We then excluded singleton OTUs and samples with a total frequency $<500$ features, and estimated beta-diversity for each dataset using robust Aitchison distances ${ }^{23}$. The final feature table for full-length rRNA operon beta-diversity analysis had 242 samples and 196 features.

Shotgun metagenomic sequencing. One round of DNA extraction was performed as above for shotgun metagenomic sequencing. Sequencing libraries were prepared using a high-throughput version of the HyperPlus library chemistry (Kapa Biosystems) miniaturized to approximately 1:10 reagent volume and optimized for nanoliter-scale liquid-handling robotics $\underline{61}$. An exhaustive, step-by-step protocol and accompanying software can be found in Sanders et al. $(2019)^{61}$. Briefly, DNA from each sample was transferred to a 384-well plate and quantified using the Quant-iT PicoGreen dsDNA Assay Kit, and then normalized to $5 \mathrm{ng}$ in $3.5 \mu \mathrm{L}$ of moleculargrade water using an Echo 550 acoustic liquid-handling robot (Labcyte, San Jose, CA, USA). For library preparation, reagents for each step (i.e., fragmentation, end repair and A-tailing, ligation, and PCR) were added in 1:10 the recommended volumes using a Mosquito HTS micropipetting robot (SPT Labtech, Tokyo, Japan). Fragmentation was performed at $37^{\circ} \mathrm{C}$ for 20 min and A-tailing at $65^{\circ} \mathrm{C}$ for $30 \mathrm{~min}$.

Sequencing adapters and barcode indices were added in two steps ${ }^{62}$. First, the Mosquito HTS robot was used to add universal adapter "stub" adapters and ligase mix to the end-repaired DNA, and the ligation reaction performed for $20^{\circ} \mathrm{C}$ for $1 \mathrm{~h}$. Adapter-ligated DNA was then cleaned-up using AMPure XP magnetic beads and a BlueCat purification robot (BlueCat Bio, Concord, Massachusetts, USA) by adding $7.5 \mu \mathrm{L}$ magnetic bead solution to the total sample volume, washing twice with $70 \% \mathrm{EtOH}$, and resuspending in $7 \mu \mathrm{L}$ molecular-grade water. Then, the Echo 
Shaffer et al. Metabolite-microbe profiles are shaped by the environment

550 robot was used to add individual i7 and i5 indices were added to adapter-ligated samples without repeating any barcodes, and by iterating the assignment of i7 to i5 indices such to minimize repeating unique i7:i5 pairs. Cleaned, adapter-ligated DNA was then amplified by adding $4.5 \mu \mathrm{L}$ of each sample to $5.5 \mu \mathrm{L}$ PCR master mix and running for 15 cycles, and then purified again using magnetic beads and the BlueCat robot. Each sample was eluted into $10 \mu \mathrm{L}$ water, and then transferred to a 384-well plate using the Mosquito HTS robot. Each library was quantified using qPCR and then pooled to equal molar fractions using the Echo 550 robot. The final pool was sequenced at Illumina on a NovaSeq6000 using S2 flow cells and 2x150-bp chemistry (Illumina, San Diego, California, USA). To increase sequence coverage for certain samples, libraries were re-pooled and a second sequencing run performed as above.

Shotgun data analysis. Raw sequence data were converted from bcl to fastq and demultiplexed to produce per-sample fastq files before processing with Atropos (v1.1.24) ${ }^{63}$ to trim universal adapter sequences, and poly-G tails introduced by the NovaSeq instrument (i.e., from use of twocolor chemistry), from reads. Trimmed reads were then mapped to the Web of Life database of microbial genomes ${ }^{64}$ to produce alignments that were used for both taxonomic and functional analysis of microbial communities.

For the taxonomic analysis, we generated a feature-table of counts of operational genomic units (OGUs) for each sample, using Woltka's (https://github.com/qiyunzhu/woltka) classify function, with per-genome alignments and default parameters. For diversity analyses, to best normalize sampling effort per sample while maintaining $\geq 75 \%$ of samples representative of the Earth's environments, we rarefied the OGU feature-table to 6,550 reads per sample. The final featuretable for diversity analyses contained 612 samples and 8,692 OGUs. For alpha-diversity, we 
Shaffer et al. Metabolite-microbe profiles are shaped by the environment

quantified three metrics, in part to see which had the strongest correlations with microbiallyrelated metabolite richness. We used the R package geiger ${ }^{65}$ to quantify weighted Faith's PD for each sample following the method of Swensen ${ }^{66}$. We used QIIME 2's diversity plugin ${ }^{22}$ to quantify richness and Faith's PD (i.e., unweighted), as well as beta-diversity (i.e., using weighted UniFrac distance) between each pair of samples. We performed PERMANOVA on that distance matrix to test for significant differences in microbial community composition across the various levels of EMPO (version 2). We then used Principal Coordinates Analysis (PCoA) and EMPeror $\underline{25}$ to visualize differences in composition among samples.

For the functional analysis, we generated a feature-table of counts of Gene Ontology (GO) Terms (i.e., for biological process, molecular function, and cellular compartment) for each sample using Woltka's collapse function, inputting per-gene alignments and with default parameters for mapping to GO Terms through MetaCyc. For subsequent analysis, we excluded singleton features and rarefied the data to 5,000 sequences per sample. The final feature-table for functional analysis included 517 samples and 3,776 features.

\section{MULTI-OMICS}

Alpha-diversity correlations. Using the alpha-diversity metrics for LC-MS/MS (i.e., richness) and shotgun metagenomic taxonomic data (i.e., richness, Faith's PD, and weighted Faith's PD), we performed correlation analysis to better understand their relationship. We used the function multilevel available in the R package correlation $^{67}$ to perform Spearman correlations for each environment (i.e., based on EMPO version 2, level 4), treating study as a random effect, and adjusting for multiple comparisons using the Benjamini-Hochberg correction. 
Shaffer et al. Metabolite-microbe profiles are shaped by the environment

Machine-learning analyses. For machine-learning analyses of LC-MS/MS and shotgun metagenomic taxonomic- and functional data, additional samples were filtered from featuretables such to exclude environments (i.e., based on EMPO version 2, level 4) with relatively low sample representation $(<10$ samples).

For the LC-MS/MS feature-table, we excluded samples in the three EMPO environments (i.e., "Animal corpus (non-saline)", "Surface (saline)", "Animal proximal gut (non-saline)"), and the final feature-table included 606 samples (covering 15 EMPO environments), and 6,588 secondary metabolites.

For the shotgun metagenomic feature-table for taxonomic analysis, we used the rarefied table described above, but further excluded samples in the five lowest-representation environments (based on EMPO version 2, level 4); the final feature-table included 598 samples (representing 14 environments), and 8,567 features (i.e., OGUs).

For the shotgun metagenomic feature-table for functional analysis, we used the rarefied table described above, but further excluded the seven lowest-representation environments; the final feature-table included 485 samples (representing 12 environments) and 2,436 features (i.e., GO terms).

For each feature-table, we trained a Random Forest classifier for predicting environments with tuned hyperparameters with a 20-time repeated group 5-fold cross-validation approach using the R package caret $^{68}$. Each dataset was split into a training set (80\%) and a testing set (20\%), with similar environmental distributions in each fold iteration for the classification of samples. To assess any batch effects on the classification of environments (i.e., potential confounding from overrepresentation of environments by single studies), the data-split in our approach was such 
Shaffer et al. Metabolite-microbe profiles are shaped by the environment

that all samples from the same study were assigned to either train- or test data sets. With this approach, we can report the study-level prediction accuracy of Random Forest classifiers. We next evaluated the prediction performance with overall accuracy quantified from a confusion matrix based on all predictions in a 100-fold cross-validation. The overall accuracy of the classifiers was calculated from the confusion matrix based on all predictions in the 100 -fold cross-validation. We evaluated how well the classifier could predict each environment by quantifying the area under the receiver operating characteristic (AUROC) and the area under the precision-recall curve (AUPRC). To identify the most important features contributing to the classification, we visualized the importance score and environment-wide prevalence and relative abundance (i.e., intensity for metabolites) of the top 32 most important features in each dataset.

Metabolite-microbe co-occurrence analysis. For co-occurrence analyses of LC-MS/MS metabolites and genomes profiled from shotgun metagenomic data, feature-tables were further filtered to only retain the 434 samples found in both datasets. For the LC-MS/MS feature-table of microbially-related secondary metabolites, we excluded 172 samples lacking shotgun metagenomics data, resulting in a final set of 6,501 metabolites. For the shotgun metagenomics feature-table for taxonomy, we excluded 150 samples lacking LC-MS/MS data, resulting in a final set of 4,120 OGUs.

We obtained co-occurrence probabilities using mmvec, which we used to predict metabolites based on microbial taxa from neural-network, compositionally-robust modeling ${ }^{69}$. The model was trained on $80 \%$ of the 434 samples, which were selected to balance environments (i.e., EMPO version 2, level 4), and used the following parameters: epochs $=200$, batch-size $=165$, learning-rate $=1 \mathrm{e}-5$, summary-interval $=1$, and with 'equalize-biplot'. We also filtered for 
Shaffer et al. Metabolite-microbe profiles are shaped by the environment

training and testing to retain only those features present in at least 10 samples (i.e., min-featurecount $=10$ ), and restricted decomposition of the co-occurrence matrix to 10 principal components $(\mathrm{PCs})$ (i.e., latent-dim $=10)$. We then calculated the Spearman correlation between the loadings of metabolites on each PC vs. (i) betas for metabolite abundances across environments from Songbird multinomial regression, (ii) loadings for metabolites on the first three axes from the RPCA ordination corresponding to clustering of samples by environment, and (iii) a vector representing the global magnitude of metabolite importance across all three axes from the RPCA biplot. We also visualized the relationships between metabolite-microbe cooccurrences and (i) by ordinating the first three PCs and coloring metabolites by their beta values (e.g., Fig. 3c).

Mantel correlations between datasets. To explore the relationships between sample-to-sample distances for any two datasets (e.g., LC-MS/MS vs. shotgun metagenomic taxonomic), we used QIIME2's diversity plugin ${ }^{22}$ to perform Mantel tests using Spearman correlations. Input distance matrices are those described above for each dataset.

\section{SUPPLEMENTARY FIGURES}


Shaffer et al. Metabolite-microbe profiles are shaped by the environment
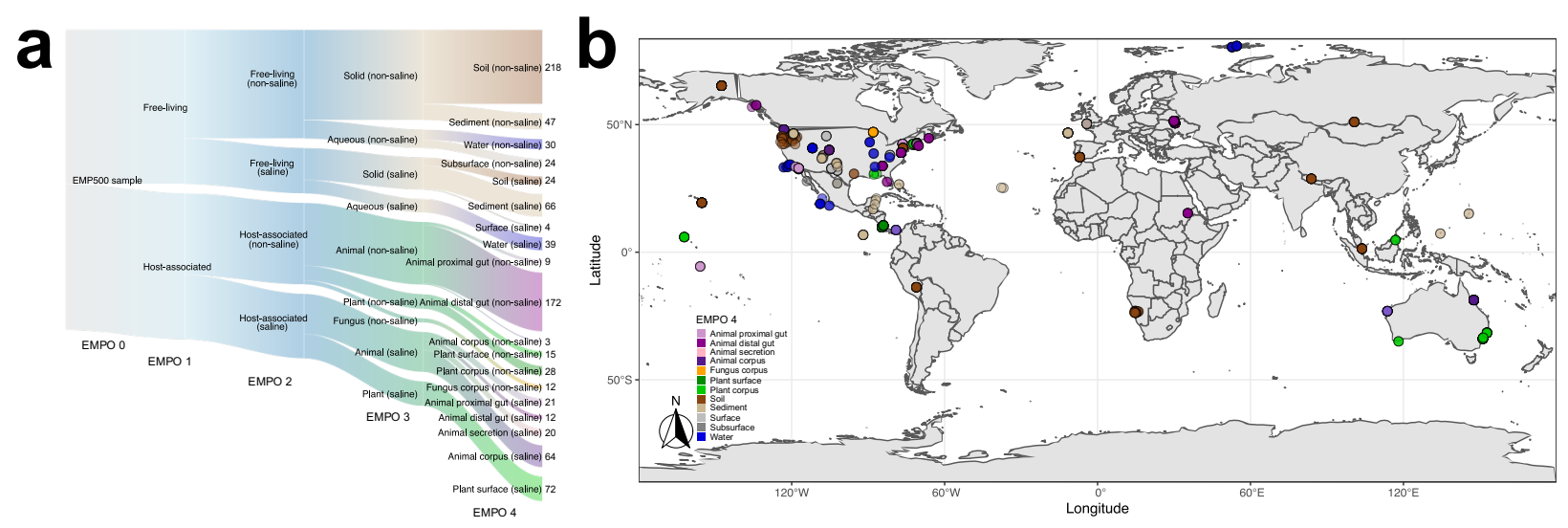

Figure S1 | a, Distribution of samples among the Earth Microbiome Project Ontology (EMPO version 2) categories. b, Geographic distribution of samples with points colored by EMPO (version 2, level 4). Extensive information about each sample set contributed is described in Table S1. 
bioRxiv preprint doi: https://doi.org/10.1101/2021.06.04.446988; this version posted November 4, 2021. The copyright holder for this preprint (which was not certified by peer review) is the author/funder. All rights reserved. No reuse allowed without permission.

Shaffer et al. Metabolite-microbe profiles are shaped by the environment

a

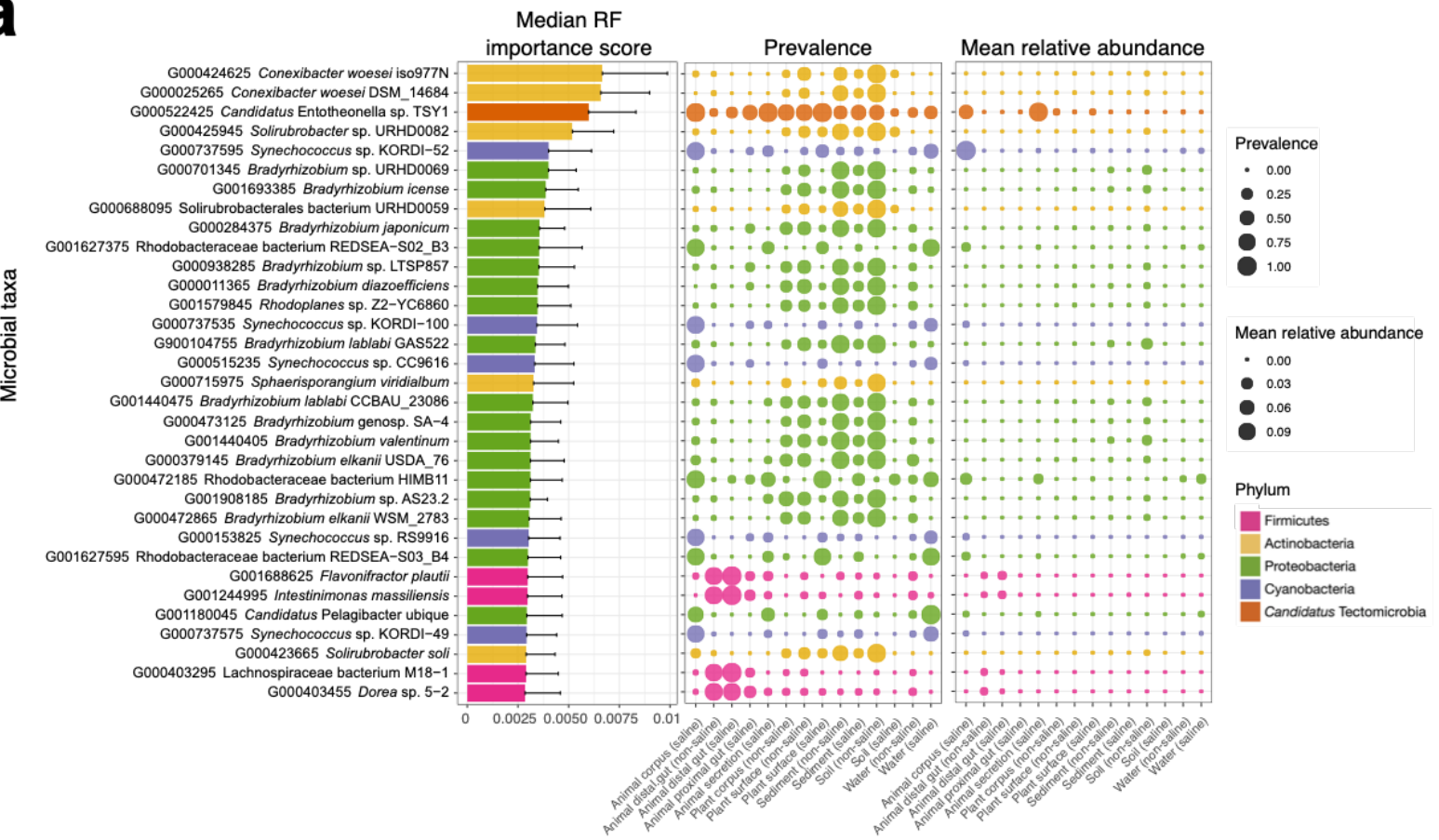

b

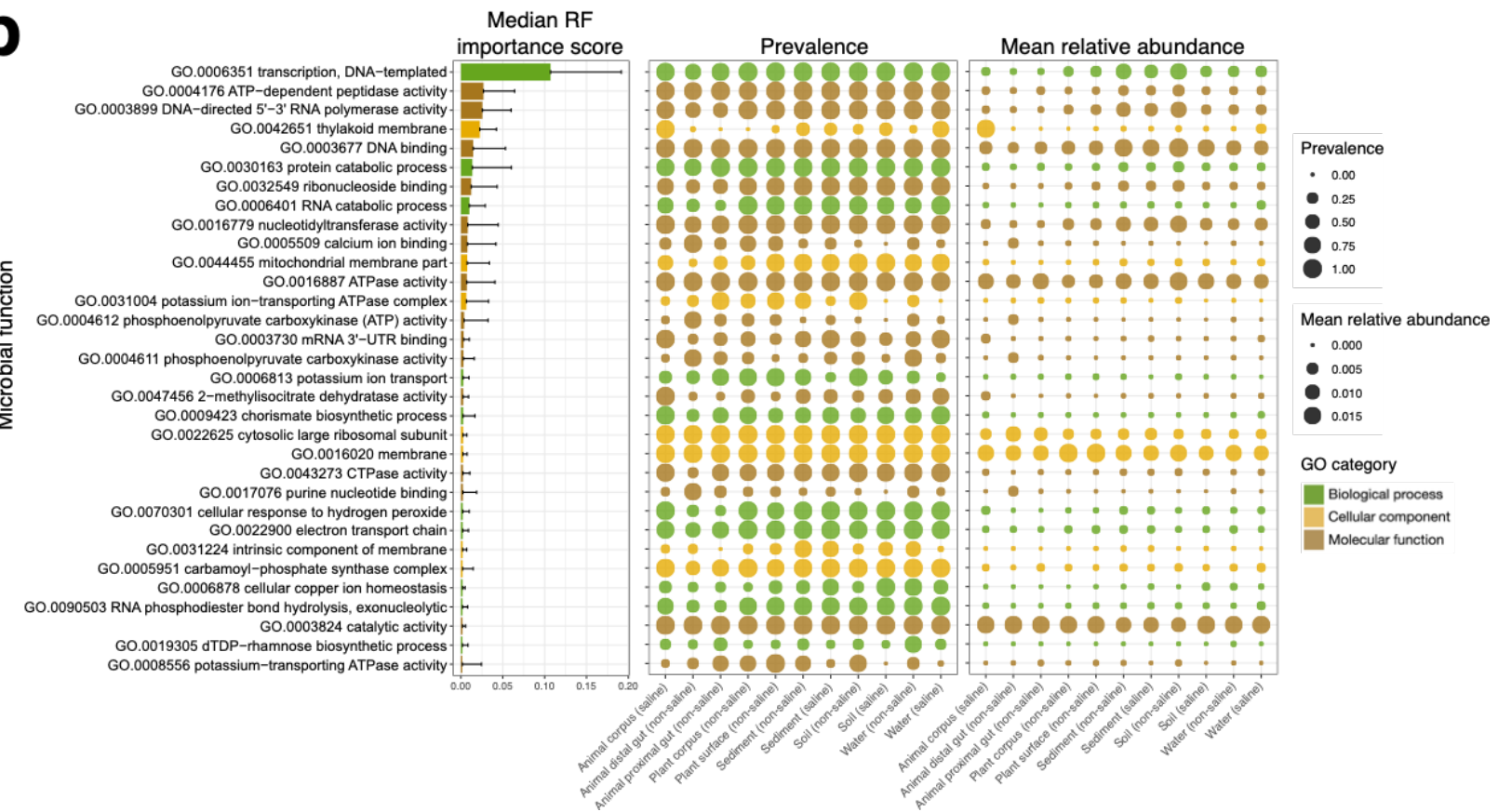

Figure S2 | Machine-learning analysis of shotgun metagenomics data highlighting the most

important microbial taxa and functions. a, The random-forest (RF) importance score,

environment-wide prevalence, and mean relative abundance for the top 32 most important

microbial taxa contributing to the separation of environments. b, The RF importance score, 
Shaffer et al. Metabolite-microbe profiles are shaped by the environment

environment-wide prevalence, and mean relative abundance for the top 32 most important microbial functions (i.e., GO Terms) contributing to the separation of environments. For both analyses, environments are described by the Earth Microbiome Project Ontology (EMPO version 2, level 4). All samples were analyzed using 20-time repeated group five-fold cross validation, where 'group' indicates the study identifier that a sample belongs to. In this approach, we split the data by 'group' such that all samples from the same study identifier can be assigned to either train or test data sets. 
bioRxiv preprint doi: https://doi.org/10.1101/2021.06.04.446988; this version posted November 4, 2021. The copyright holder for this preprint (which was not certified by peer review) is the author/funder. All rights reserved. No reuse allowed without permission.

Shaffer et al. Metabolite-microbe profiles are shaped by the environment
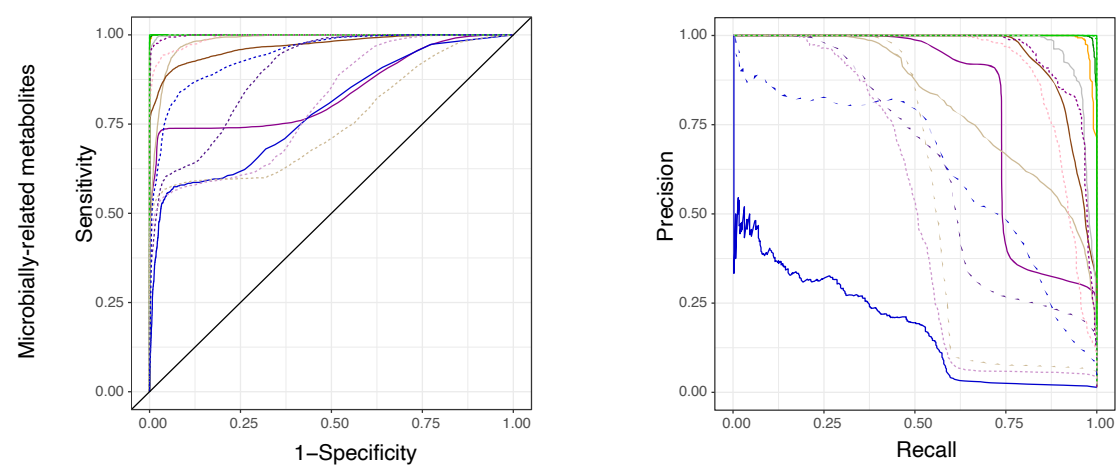

Environment

.... Animal corpus (saline): AUROC $=0.9$; AUPRC $=0.64$

- Animal distal gut (non-saline): AUROC $=0.84 ;$ AUPRC $=0.81$

.... Animal distal gut (saline): AUROC=1; $A \cup P R C=0.96$

-...'Animal proximal.gut (saline): AUROC=0.81; AUPRC=0.51

-1. Animal secretion (saline): AUROC=0.99; $\mathrm{AUPRC}=0.91$

- Fungus corpus (non-saline): AUROC=1; AUPRC=1

- Plant corpus (non-saline): AUROC=1; AUPRC=1

- Plant surface (non-saline): $A U R O C=1 ;$ AUPRC= 1

.... Plant surface (saline): $A U R O C=1 ;$ AUPRC $=1$

— Sediment (non-saline): AUROC=0.98; AUPRC=0.82

wn.. Sediment (saline): AUROC $=0.75$; AUPRC $=0.58$

- Soil (non-saline): AUROC=0.97; AUPRC=0.95

- Subsurface (non-saline): AUROC=1; AUPRC=0.97

- Water (non-saline): AUROC=0.79; AUPRC=0.18

... Water (saline): AUROC=0.95; AUPRC=0.64
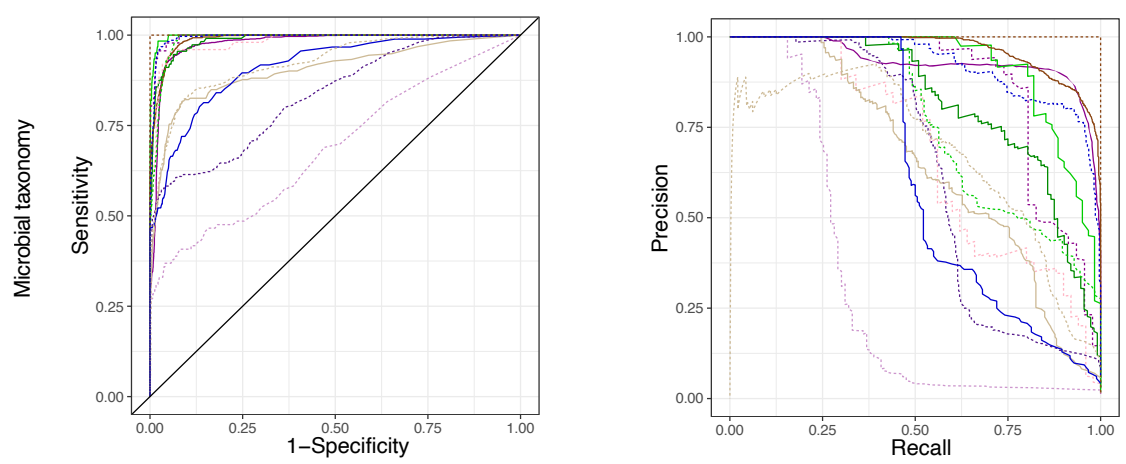

Environment

- Animal distal.gut (non-saline): AUROC=0.98; AUPRC=0.93

..." Animal distal.gut (saline): AUROC=1; AUPRC=0.87

nun Animal proximal.gut (saline): AUROC=0.68; AUPRC=0.31

(unul Animal secretion (saline): AUROC $=0.98 ;$ AUPRC $=0.68$

- Plant corpus (non-saline): AUROC=0.99; AUPRC $=0.82$

- Plant surface (non-saline): AUROC=1; AUPRC=0.92

nus! Plant surface (saline): $\mathrm{AUROC}=0.99$; $\mathrm{AUPRC}=0.76$

- Sediment (non-saline): AUROC=0.9; AUPRC=0.65

n.... Sediment (saline): AUROC=0.92; AUPRC=0.68

- Soil (non-saline): AUROC=0.99; AUPRC=0.96

.... Soil (saline): AUROC=1; AUPRC=1

- Water (non-saline): AUROC $=0.91 ;$ AUPRC $=0.61$

... Water (saline): AUROC=0.99; AUPRC $=0.92$
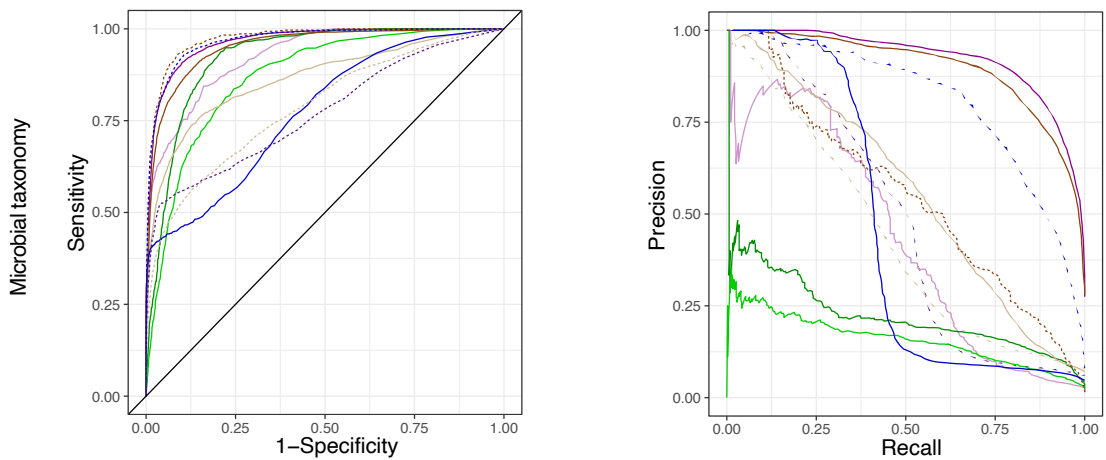

Environment

1.". Animal corpus (saline): $A$ UROC $=0.77 ;$ AUPRC $=0.48$

- Animal distal gut (non-saline): AUROC $=0.97 ;$ AUPRC $=0.92$

- Animal proximal gut (non-saline): $A U R O C=0.93 ;$ AUPRC $=0.43$

- Plant corpus (non-saline): $A \cup R O C=0.92 ; A U P R C=0.22$

- Plant surface (non-saline): AUROC $=0.87 ;$ AUPRC $=0.16$

- Sediment (non-saline): AUROC $=0.86 ;$ AUPRC $=0.56$

"IIII. Sediment (saline): $A U R O C=0.79 ;$ AUPRC $=0.43$

- Soil (non-saline): AUROC $=0.95 ;$ AUPRC $=0.9$

I". Soil (saline): $A U R O C=0.97 ;$ AUPRC $=0.55$

- Water (non-saline): AUROC $=0.77 ;$ AUPRC $=0.45$

I.. Water (saline): AUROC $=0.97 ;$ AUPRC $=0.81$

Figure S3 | Machine-learning analysis of LC-MS/MS metabolomics and shotgun

metagenomics data highlighting per-environment classification accuracy. a, Receiver

operating characteristic curves (and AUROC) and precision-recall curves (and AUPRC)

illustrating classification accuracy of the random forest model across all environments based on

microbial taxonomic (i.e., OGU) profiles. b, Receiver operating characteristic curves (and

AUROC) and precision-recall curves (and AUPRC) illustrating classification accuracy of the

random forest model across all environments based on microbial functional (i.e., GO terms) 
Shaffer et al. Metabolite-microbe profiles are shaped by the environment

profiles. For both analyses, environments are described by the Earth Microbiome Project

Ontology (EMPO version 2, level 4). All samples were analyzed using 20-time repeated group

five-fold cross validation, where 'group' indicates the study identifier that a sample belongs to.

In this approach, we split the data by 'group' such that all samples from the same study identifier can be assigned to either train or test data sets. 
bioRxiv preprint doi: https://doi.org/10.1101/2021.06.04.446988; this version posted November 4, 2021. The copyright holder for this preprint (which was not certified by peer review) is the author/funder. All rights reserved. No reuse allowed without permission.

Shaffer et al. Metabolite-microbe profiles are shaped by the environment

\section{SUPPLEMENTARY TABLES}


Table S1. Study metadata, including study identifiers, principal investigator, title, sampling method, number of samples, environmental package, EMPO level 3 (version 1), and EMPO level 4 (version 2) for each study.

\begin{tabular}{|c|c|c|c|c|c|c|c|}
\hline emp500_study_id & emp500_principal_investigator & emp500_title & sampling_method & number_of_samples & environmental_package & empo_3 & empo_v2_4 \\
\hline 1 & Kshtrika & Biota oil & bulk & 14 & misc environment & Subsurface (non-saline) & Subsurface (non-saline) \\
\hline 2 & Berry & Postglacial pond sediment profiles & bulk & 20 & sediment & Sediment (non-saline) & Sediment (non-saline) \\
\hline 3 & MacRae-Crerar & Mongolia soils (NSF PIRE) & bulk & 12 & soil & Soil (non-saline) & Soil (non-saline) \\
\hline 5 & Myrold & Tree-associated soils & bulk & 12 & soil & Soil (non-saline) & Soil (non-saline) \\
\hline 9 & Zaneveld & Scleractinian corals & fractionated & 22 & host-associated, water & Animal secretion, Water (saline) & Animal secretion, Water (saline) \\
\hline 18 & Thomas & Ocean sponges & fractionated & 57 & host-associated & Animal corpus & Animal corpus (saline) \\
\hline 19 & Thomas & Australian algae & swab & 64 & host-associated & Plant surface & Plant surface (saline) \\
\hline 21 & McMahon & Bioreactors for wastewater and anammox & bulk & 17 & wastewater/sludge & Water (non-saline) & Water (non-saline) \\
\hline 23 & Shade & Centralia soil & bulk & 10 & soil & Soil (non-saline) & Soil (non-saline) \\
\hline 26 & Stewart & Marine oxygen minimum zone water & filter & 8 & water & Water (saline) & Water (saline) \\
\hline 27 & King & Puhimau Hawai'i volcanic soil & bulk & 21 & soil & Soil (non-saline) & Soil (non-saline) \\
\hline 33 & Mayer & West Texas playa & bulk & 7 & sediment & Sediment (non-saline) & Sediment (non-saline) \\
\hline 34 & Mayer & Animas watershed & bulk & 10 & sediment & Sediment (non-saline) & Sediment (non-saline) \\
\hline 36 & Stegen & Permafrost soil & bulk & 15 & soil & Soil (non-saline) & Soil (non-saline) \\
\hline 37 & Stegen & Hyporheic sediment & bulk & 10 & sediment & Sediment (non-saline) & Sediment (non-saline) \\
\hline 38 & Stegen & Active layer soil & bulk & 18 & soil & Soil (non-saline) & Soil (non-saline) \\
\hline 39 & Smith & Palmyra algae & bulk or swab & 8 & host-associated & Plant surface & Plant surface (saline) \\
\hline 40 & Metcalf & Mouse decomposition soil & bulk & 14 & soil & Soil (non-saline) & Soil (non-saline) \\
\hline 42 & Palenik & San Diego seawater & filter & 20 & water & Water (saline) & Water (saline) \\
\hline 43 & Jensen & Ocean sediments & bulk & 19 & sediment & Sediment (saline) & Sediment (saline) \\
\hline 45 & Roy-Chowdhury & Spanish hypersaline sandy soils & bulk & 24 & soil & Soil (non-saline) & Soil (non-saline) \\
\hline 46 & Makhalanyane & Namibia gravel plain gradient soils & bulk & 10 & soil & Soil (non-saline) & Soil (non-saline) \\
\hline 47 & Makhalanyane & Namibia dune aridity gradient soils & bulk & 9 & soil & Soil (non-saline) & Soil (non-saline) \\
\hline 50 & Girguis & Whale feces & bulk & 12 & host-associated & Animal distal gut & Animal distal gut (saline) \\
\hline 51 & Song & Captive terrestrial mammal feces & bulk & 20 & host-associated & Animal distal gut & Animal distal gut (non-saline) \\
\hline 52 & Song & Captive herp feces & bulk & 15 & host-associated & Animal distal gut & Animal distal gut (non-saline) \\
\hline 53 & Song & Captive bird feces & bulk & 15 & host-associated & Animal distal gut & Animal distal gut (non-saline) \\
\hline 54 & Sandin & Fish gut contents & bulk & 15 & host-associated & Animal proximal gut & Animal proximal gut (saline) \\
\hline 56 & Schmidt & Peru and Nepal alpine soils & bulk & 26 & soil & Soil (non-saline) & Soil (non-saline) \\
\hline 58 & Tucker & Coal and coal-associated water & filter & 16 & misc environment & Subsurface (non-saline) & Subsurface (non-saline) \\
\hline 59 & Myrold & Oregon soils & bulk & 22 & soil & Soil (non-saline) & Soil (non-saline) \\
\hline 62 & Pinto & Costa Rican leaf-cutter ant soils & bulk & 24 & soil & Soil (non-saline) & Soil (non-saline) \\
\hline 63 & Pinto & Costa Rican leaf-cutter ant and bee material & bulk & 28 & host-associated & Plant corpus & Plant corpus (non-saline) \\
\hline 65 & Angenent & Rumen animals and bioreactor & bulk & 9 & host-associated & Animal proximal gut & Animal proximal gut (non-saline) \\
\hline 68 & Bittleston & Pitcher plant fluids & bulk & 15 & plant-associated & Plant surface & $\begin{array}{l}\text { Plant surface (non-saline) } \\
\text { Pantion }\end{array}$ \\
\hline 71 & Seedorf & Singapore plant-associated soils & bulk & 14 & soil & Soil (non-saline) & Soil (non-saline) \\
\hline 72 & Distel & Shipworm tissue and wood & fractionated & 15 & host-associated & $\begin{array}{l}\text { Animal corpus, Animal proximal gut, } \\
\text { Surface (non-saline) }\end{array}$ & $\begin{array}{l}\text { Animal corpus, Animal proximal gut, } \\
\text { Surface (non-saline) }\end{array}$ \\
\hline 74 & Bowen & Massachusetts salt marsh sediments & bulk & 16 & sediment & Sediment (saline) & Sediment (saline) \\
\hline 75 & Song & Captive aquatic mammal feces & bulk & 5 & host-associated & Animal distal gut & Animal distal gut (non-saline) \\
\hline 76 & Minich & Livestock feces & bulk & 6 & host-associated & Animal distal gut & Animal distal gut (non-saline) \\
\hline 77 & Tait & English Channel water & filter & 9 & water & Water (saline) & Water (saline) \\
\hline 78 & Tait & English Channel sediment & bulk & 3 & sediment & Sediment (saline) & Sediment (saline) \\
\hline 81 & Metcalf & Mouse decomposition skin & bulk & 3 & host-associated & Animal corpus & Animal corpus (non-saline) \\
\hline 82 & Uren & Michigan forest lichen & bulk & 12 & plant-associated & Fungus corpus & Fungus corpus (non-saline) \\
\hline 84 & Rohwer & Franz Josef Land glacier soils and water & bulk & 15 & soil, water & Soil (non-saline), Water (non-saline) & Soil (non-saline), Water (non-saline) \\
\hline 85 & Rohwer & Iberia and Guatemala deep-sea sediments & bulk & 27 & sediment & Sediment (saline) & Sediment (saline) \\
\hline 86 & Rohwer & Mexico microbial mats & bulk & 3 & microbial matbiofilm, sediment & Surface (saline), Sediment (saline) & Surface (saline), Sediment (saline) \\
\hline 88 & Mousseau & Chernobyl vole feces & bulk & 112 & host-associated & Animal distal gut & Animal distal gut (non-saline) \\
\hline
\end{tabular}


Shaffer et al. Metabolite-microbe profiles are shaped by the environment

Note: Scientific justification and metadata columns for each study are included in the version of the table here and will be included with the final Supplementary Material, but were excluded here due to space limitations. 
bioRxiv preprint doi: https://doi.org/10.1101/2021.06.04.446988; this version posted November 4, 2021. The copyright holder for this preprint (which was not certified by peer review) is the author/funder. All rights reserved. No reuse allowed without permission.

Shaffer et al. Metabolite-microbe profiles are shaped by the environment

Table S2. The top 10 ranked microbially-related metabolites for each environment, described using the Earth Microbiome Project Ontology (EMPO version 2, level 4). Data are from

Songbird multinomial regression (model: composition $=$ EMPO version 2, level 4; pseudo- $Q^{2}=$ 0.21). Metabolites in bold font are those also identified to be strongly associated with RPCA

axes (Fig. 2c, Table S3), those in bold, underlined font also strongly separated environments in machine-learning analysis (Table S4), and those in bold, underlined, italic font identified as important in all three analyses.

\begin{tabular}{|c|c|c|c|c|c|c|}
\hline Environment & Feature rank & Feature ID & Molecular formula & Pathway & Superclass & Class \\
\hline Animal corpus (saline) & 1 & 23235 & $5 \mathrm{C} 27 \mathrm{H} 40 \mathrm{O} 2$ & Terpenoids & Sesterterpenoids & Not annotated \\
\hline Animal corpus (saline) & 2 & 41006 & 6 Not annotated & Not annotated & Not annotated & Not annotated \\
\hline Animal corpus (saline) & 3 & 28910 & O C27H38 & Terpenoids & Steroids & Cholestane steroids \\
\hline Animal corpus (saline) & 4 & 23136 & $6 \mathrm{C} 25 \mathrm{H} 32 \mathrm{O} 3$ & Terpenoids & Cyclic polyketides & Not annotated \\
\hline Animal corpus (saline) & 5 & 21140 & $0 \mathrm{C} 29 \mathrm{H} 46 \mathrm{O} 4$ & Terpenoids & Triterpenoids & Not annotated \\
\hline Animal corpus (saline) & 6 & 3559 & 9 Not annotated & Not annotated & Not annotated & Not annotated \\
\hline Animal corpus (saline) & 7 & 23517 & 7 Not annotated & Not annotated & Not annotated & Not annotated \\
\hline Animal corpus (saline) & 8 & 41001 & 1 Not annotated & Not annotated & Not annotated & Not annotated \\
\hline Animal corpus (saline) & 9 & 3955 & 5 Not annotated & Not annotated & Not annotated & Not annotated \\
\hline Animal corpus (saline) & 10 & 39515 & 5 Not annotated & Not annotated & Not annotated & Not annotated \\
\hline Animal distal gut (non-saline) & 1 & 25552 & 2 C24H3804 & Terpenoids & Steroids & Cholane steroids \\
\hline Animal distal gut (non-saline) & 2 & 7792 & $2 \mathrm{C} 24 \mathrm{H} 38 \mathrm{O} 4$ & Terpenoids & Steroids & Cholane steroids \\
\hline Animal distal gut (non-saline) & 3 & 25862 & $2 \mathrm{C} 27 \mathrm{H} 42 \mathrm{O} 7$ & Terpenoids & Steroids & Ecdysteroids \\
\hline Animal distal gut (non-saline) & 4 & 51039 & $9 \mathrm{C} 27 \mathrm{H} 44 \mathrm{O} 3$ & Terpenoids & Steroids & Cholestane steroids \\
\hline Animal distal gut (non-saline) & 5 & 2081 & $1 \mathrm{C} 15 \mathrm{H} 1004$ & Shikimates and Phenylpropanoids & Isoflavonoids & Isoflavones \\
\hline Animal distal gut (non-saline) & 6 & 1236 & $6 \mathrm{C} 18 \mathrm{H} 3405$ & Fatty acids & Octadecanoids & Other Octadecanoids \\
\hline Animal distal gut (non-saline) & 7 & 50823 & $3 \mathrm{C} 26 \mathrm{H} 45 \mathrm{NO} 06 \mathrm{~S}$ & Terpenoids & Steroids & Cholane steroids \\
\hline Animal distal gut (non-saline) & 8 & 22299 & $9 \mathrm{C} 29 \mathrm{H} 46 \mathrm{O} 2$ & Terpenoids & Meroterpenoids & Prenyl quinone meroterpenoids \\
\hline Animal distal gut (non-saline) & 9 & 2826 & 6 С19H3404 & Fatty acids & Octadecanoids & Other Octadecanoids \\
\hline Animal distal gut (non-saline) & 10 & 12107 & $7 \mathrm{C} 24 \mathrm{H} 41 \mathrm{NO} 3$ & Terpenoids & Steroids & Cholane steroids \\
\hline Animal distal gut (saline) & 1 & 29157 & 7 Not annotated & Not annotated & Not annotated & Not annotated \\
\hline Animal distal gut (saline) & 2 & 38117 & 7 C4OH52O4 & Terpenoids & Carotenoids (C40) & Carotenoids $(C 40, \beta-\beta)$ \\
\hline Animal distal gut (saline) & 3 & 7942 & $2 \mathrm{C} 17 \mathrm{H} 24 \mathrm{~N} 2 \mathrm{O} 4$ & Alkaloids & Ornithine alkaloids & Not annotated \\
\hline Animal distal gut (saline) & 4 & 53059 & $9 \mathrm{C} 15 \mathrm{H} 18 \mathrm{BrO}$ & Terpenoids & Sesquiterpenoids & Not annotated \\
\hline Animal distal gut (saline) & 5 & 30947 & 7 C7H9NO2 & Shikimates and Phenylpropanoids & Not annotated & Shikimic acids and derivatives \\
\hline Animal distal gut (saline) & 6 & 20600 & 0 Not annotated & Not annotated & Not annotated & Not annotated \\
\hline Animal distal gut (saline) & 7 & 50368 & 8 C27H31N303 & Alkaloids & Tryptophan alkaloids & Not annotated \\
\hline Animal distal gut (saline) & 8 & 52381 & 1 Not annotated & Not annotated & Not annotated & Not annotated \\
\hline Animal distal gut (saline) & 9 & 50979 & $9 \mathrm{C} 24 \mathrm{H} 3602 \mathrm{~S}$ & Terpenoids & Steroids & Cholane steroids \\
\hline Animal distal gut (saline) & 10 & 12107 & $7 \mathrm{C} 24 \mathrm{H} 41 \mathrm{NO} 3$ & Terpenoids & Steroids & Cholane steroids \\
\hline Animal proximal gut (saline) & 1 & 46142 & $2 \mathrm{C} 19 \mathrm{H} 39 \mathrm{NO} 2 \mathrm{~S}$ & Fatty acids & Fatty Acids and Conjugates & Not annotated \\
\hline Animal proximal gut (saline) & 2 & 50500 & 0 Not annotated & Not annotated & Not annotated & Not annotated \\
\hline Animal proximal gut (saline) & 3 & 46161 & 1 C19H39NO2S & Fatty acids & Fatty Acids and Conjugates & Not annotated \\
\hline Animal proximal gut (saline) & 4 & 50865 & 5 Not annotated & Not annotated & Not annotated & Not annotated \\
\hline Animal proximal gut (saline) & 5 & 8409 & $9 \mathrm{C} 22 \mathrm{H} 35 \mathrm{NO} 2$ & Fatty acids & Fatty amides & $\mathrm{N}$-acyl ethanolamines (endocannabinoids) \\
\hline Animal proximal gut (saline) & 6 & 15710 & 0 Not annotated & Not annotated & Not annotated & Not annotated \\
\hline Animal proximal gut (saline) & 7 & 19189 & 9 Not annotated & Not annotated & Not annotated & Not annotated \\
\hline Animal proximal gut (saline) & 8 & 44429 & 9 Not annotated & Not annotated & Not annotated & Not annotated \\
\hline Animal proximal gut (saline) & 9 & 47998 & 8 Not annotated & Not annotated & Not annotated & Not annotated \\
\hline Animal proximal gut (saline) & 10 & 49293 & 3 C18H36N4O4 & Amino acids and Peptides & Small peptides & Not annotated \\
\hline Animal secretion (saline) & 1 & 24213 & $3 \mathrm{C} 25 \mathrm{H} 40 \mathrm{O} 3$ & Fatty acids & Glycerolipids & Monoacylglycerols \\
\hline Animal secretion (saline) & 2 & 46948 & $8 \mathrm{C} 2 \mathrm{OH} 35 \mathrm{~N} 5 \mathrm{O} 13$ & Carbohydrates & Polyols & Amino cyclitols \\
\hline Animal secretion (saline) & 3 & 24185 & 5 C2OH33 $\mathrm{ClO} 4$ & Polyketides & Not annotated & Not annotated \\
\hline Animal secretion (saline) & 4 & 10248 & $8 \mathrm{C} 24 \mathrm{H} 41 \mathrm{NOS}$ & Not annotated & Steroids & Not annotated \\
\hline Animal secretion (saline) & 5 & 16936 & 6 С33H52O13 & Fatty acids & Glycerolipids & Glycosyldiacylglycerols \\
\hline Animal secretion (saline) & 6 & 24147 & $7 \mathrm{C} 25 \mathrm{H} 4003$ & Terpenoids & Steroids & Cholane steroids \\
\hline Animal secretion (saline) & 7 & 40413 & $3 \mathrm{C} 22 \mathrm{H} 46 \mathrm{NO} 08 \mathrm{P}$ & Fatty acids & Glycerophospholipids & Glycerophosphates \\
\hline Animal secretion (saline) & 8 & 44580 & $\mathrm{O}$ C11H21N3O & Alkaloids & Small peptides & Dipeptides \\
\hline Animal secretion (saline) & 9 & 22091 & $1 \mathrm{C} 28 \mathrm{H} 5009$ & Polyketides & Polyethers & Not annotated \\
\hline Animal secretion (saline) & 10 & 46073 & $3 \mathrm{C} 10 \mathrm{H} 17 \mathrm{~N}$ & Terpenoids & Monoterpenoids & Not annotated \\
\hline Fungus corpus (non-saline) & 1 & 20438 & $8 \mathrm{C} 18 \mathrm{H} 1007$ & Shikimates and Phenylpropanoids & Flavonoids & Flavonols \\
\hline Fungus corpus (non-saline) & 2 & 16650 & O C18H12O8 & Shikimates and Phenylpropanoids & Flavonoids & Flavonols \\
\hline Fungus corpus (non-saline) & 3 & 16117 & 7 C25H43NO9 & Fatty acids & Glycerolipids & Glycosyldiacylglycerols \\
\hline Fungus corpus (non-saline) & 4 & 16952 & 2 Not annotated & Not annotated & Not annotated & Not annotated \\
\hline Fungus corpus (non-saline) & 5 & 16752 & 2 C18H30Cl2O2 & Fatty acids & Fatty Acids and Conjugates & Not annotated \\
\hline Fungus corpus (non-saline) & 6 & 16681 & $1 \mathrm{C} 18 \mathrm{H} 12 \mathrm{O} 6$ & Shikimates and Phenylpropanoids & Flavonoids & Not annotated \\
\hline Fungus corpus (non-saline) & 7 & 16632 & $2 \mathrm{C} 17 \mathrm{H} 10 \mathrm{O} 6$ & Polyketides & Xanthones & Methyl xanthones \\
\hline Fungus corpus (non-saline) & 8 & 16615 & $5 \mathrm{C} 18 \mathrm{H} 12 \mathrm{O} 7$ & Shikimates and Phenylpropanoids & Not annotated & Not annotated \\
\hline Fungus corpus (non-saline) & 9 & 16743 & $3 \mathrm{C} 18 \mathrm{H} 12 \mathrm{O} 8$ & Shikimates and Phenylpropanoids & Flavonoids & Flavonols \\
\hline Fungus corpus (non-saline) & 10 & 15767 & $7 \mathrm{C} 2 \mathrm{OH} 34 \mathrm{O} 2$ & Fatty acids & Fatty Acids and Conjugates & Not annotated \\
\hline
\end{tabular}


bioRxiv preprint doi: https://doi.org/10.1101/2021.06.04.446988; this version posted November 4, 2021. The copyright holder for this preprint (which was not certified by peer review) is the author/funder. All rights reserved. No reuse allowed without permission.

\section{Shaffer et al. Metabolite-microbe profiles are shaped by the environment}

\section{Table S2. (continued)}

\begin{tabular}{|c|c|c|c|c|c|c|}
\hline Environment & Feature rank & Feature ID & Molecular formula & Pathway & Superclass & Class \\
\hline Fungus corpus (non-saline) & 1 & 2043 & $8 \mathrm{C} 18 \mathrm{H} 1007$ & Shikimates and Phenylpropanoids & Flavonoids & Flavonols \\
\hline Fungus corpus (non-saline) & 2 & 1665 & $0 \mathrm{C} 18 \mathrm{H} 1208$ & Shikimates and Phenylpropanoids & Flavonoids & Flavonols \\
\hline Fungus corpus (non-saline) & 3 & 1611 & 7 C25H43NO9 & Fatty acids & Glycerolipids & Glycosyldiacylglycerols \\
\hline Fungus corpus (non-saline) & 4 & 1695 & 2 Not annotated & Not annotated & Not annotated & Not annotated \\
\hline Fungus corpus (non-saline) & 5 & 1675 & $2 \mathrm{C} 18 \mathrm{H} 30 \mathrm{Cl} 2 \mathrm{O} 2$ & Fatty acids & Fatty Acids and Conjugates & Not annotated \\
\hline Fungus corpus (non-saline) & 6 & 1668 & $1 \mathrm{C} 18 \mathrm{H} 12 \mathrm{O} 6$ & Shikimates and Phenylpropanoids & Flavonoids & Not annotated \\
\hline Fungus corpus (non-saline) & 7 & 1663 & $2 \mathrm{C} 17 \mathrm{H} 10 \mathrm{O} 6$ & Polyketides & Xanthones & Methyl xanthones \\
\hline Fungus corpus (non-saline) & 8 & 1661 & $5 \mathrm{C} 18 \mathrm{H} 12 \mathrm{O} 7$ & Shikimates and Phenylpropanoids & Not annotated & Not annotated \\
\hline Fungus corpus (non-saline) & 9 & 1674 & $3 \mathrm{C} 18 \mathrm{H} 12 \mathrm{O} 8$ & Shikimates and Phenylpropanoids & Flavonoids & Flavonols \\
\hline Fungus corpus (non-saline) & 10 & 1576 & $7 \mathrm{C} 2 \mathrm{OH} 3402$ & Fatty acids & Fatty Acids and Conjugates & Not annotated \\
\hline Plant corpus (non-saline) & 1 & 2467 & 0 С $33 \mathrm{H} 4004$ & Polyketides & Meroterpenoids & Not annotated \\
\hline Plant corpus (non-saline) & 2 & 2467 & $8 \mathrm{C} 13 \mathrm{H} 16 \mathrm{BNO} 7$ & Amino acids and Peptides & Not annotated & Not annotated \\
\hline Plant corpus (non-saline) & 3 & 2469 & 2 C33H48O4 & Polyketides & Meroterpenoids & Not annotated \\
\hline Plant corpus (non-saline) & 4 & 739 & $5 \mathrm{C} 22 \mathrm{H} 3003$ & Terpenoids & Meroterpenoids & Cannabinoids \\
\hline Plant corpus (non-saline) & 5 & 2468 & 4 Not annotated & Not annotated & Not annotated & Not annotated \\
\hline Plant corpus (non-saline) & 6 & 2467 & 7 C24H2604 & Polyketides & Phloroglucinols & Acyl phloroglucinols \\
\hline Plant corpus (non-saline) & 7 & 3011 & $1 \mathrm{C} 27 \mathrm{H} 42 \mathrm{O} 6$ & Terpenoids & Steroids & Ecdysteroids \\
\hline Plant corpus (non-saline) & 8 & 2486 & $6 \mathrm{C19H} 1805$ & Polyketides & Phloroglucinols & Not annotated \\
\hline Plant corpus (non-saline) & 9 & 2628 & $5 \mathrm{C} 28 \mathrm{H} 42$ & Terpenoids & Steroids & Vitamin $\mathrm{D} 2$ and derivatives \\
\hline Plant corpus (non-saline) & 10 & 3173 & 5 C24H39N508 & Amino acids and Peptides & Small peptides & Tripeptides \\
\hline Plant surface (non-saline) & 1 & 1132 & $6 \mathrm{C} 24 \mathrm{H} 41 \mathrm{NO} 4$ & Terpenoids & Steroids & Cholane steroids \\
\hline Plant surface (non-saline) & 2 & 3640 & 0 С $32 \mathrm{H} 5404$ & Polyketides & Meroterpenoids & Not annotated \\
\hline Plant surface (non-saline) & 3 & 2077 & $5 \mathrm{C} 2 \mathrm{OH} 41 \mathrm{NO} 4$ & Fatty acids & Not annotated & Other Octadecanoids \\
\hline Plant surface (non-saline) & 4 & 494 & 9 С13н100 & Shikimates and Phenylpropanoids & Flavonoids & Chalcones \\
\hline Plant surface (non-saline) & 5 & 3075 & $1 \mathrm{C9H} 15 \mathrm{NO} 4$ & Polyketides & Cyclic polyketides & 2-pyrone derivatives \\
\hline Plant surface (non-saline) & 6 & 5118 & $8 \mathrm{C} 21 \mathrm{H} 24 \mathrm{O} 3$ & Terpenoids & Diterpenoids & Tetracyclic diterpenoids \\
\hline Plant surface (non-saline) & 7 & 20098 & $8 \mathrm{C} 7 \mathrm{H} 8 \mathrm{NO} 4$ & Amino acids and Peptides & Small peptides & Aminoacids \\
\hline Plant surface (non-saline) & 8 & 4516 & $9 \mathrm{C} 26 \mathrm{H} 41 \mathrm{NO} 8$ & Alkaloids & Not annotated & Not annotated \\
\hline Plant surface (non-saline) & 9 & 3886 & 6 Not annotated & Not annotated & Not annotated & Not annotated \\
\hline Plant surface (non-saline) & 10 & 2181 & 5 C3OH5ON6O4 & Amino acids and Peptides & Oligopeptides & Lipopeptides \\
\hline Plant surface (saline) & 1 & 5561 & 4 C8H13N3O & Alkaloids & Nicotinic acid alkaloids & Pyridine alkaloids \\
\hline Plant surface (saline) & 2 & 5597 & 5 C22H41N308 & Polyketides & Not annotated & Not annotated \\
\hline Plant surface (saline) & 3 & 5648 & 5 Not annotated & Not annotated & Not annotated & Not annotated \\
\hline Plant surface (saline) & 4 & 5632 & 4 C21H37N307 & Polyketides & Not annotated & Not annotated \\
\hline Plant surface (saline) & 5 & 3440 & $7 \mathrm{C1OH} 19 \mathrm{~N} 3 \mathrm{O} 2$ & Alkaloids & Not annotated & Primary amides \\
\hline Plant surface (saline) & 6 & 56318 & $8 \mathrm{C} 10 \mathrm{H} 17 \mathrm{~N} 3 \mathrm{O}$ & Alkaloids & Monoterpenoids & Pyridine alkaloids \\
\hline Plant surface (saline) & 7 & 3465 & $2 \mathrm{C} 10 \mathrm{H} 19 \mathrm{~N} 3 \mathrm{O} 2$ & Alkaloids & Not annotated & Primary amides \\
\hline Plant surface (saline) & 8 & 5654 & $8 \mathrm{C} 23 \mathrm{H} 4008$ & Fatty acids & Linear polyketides & Open-chain polyketides \\
\hline Plant surface (saline) & 9 & 5601 & 4 C22H39N3O7 & Amino acids and Peptides & Not annotated & Not annotated \\
\hline Plant surface (saline) & 10 & 5649 & $6 \mathrm{C} 21 \mathrm{H} 41 \mathrm{~N} 3 \mathrm{O} 7$ & Polyketides & Linear polyketides & Open-chain polyketides \\
\hline Sediment (non-saline) & 1 & 1502 & $4 \mathrm{C} 13 \mathrm{H} 24 \mathrm{~N} 607$ & Carbohydrates & Not annotated & Not annotated \\
\hline Sediment (non-saline) & 2 & 470 & 2 С9H1605 & Fatty acids & Glycerolipids & Not annotated \\
\hline Sediment (non-saline) & 3 & 2822 & $1 \mathrm{C} 36 \mathrm{H} 5408$ & Terpenoids & Diterpenoids & Tigliane diterpenoids \\
\hline Sediment (non-saline) & 4 & 803 & $0 \mathrm{C} 2 \mathrm{OH} 30$ & Terpenoids & Steroids & Not annotated \\
\hline Sediment (non-saline) & 5 & 2137 & $9 \mathrm{C} 25 \mathrm{H} 26 \mathrm{O}$ & Shikimates and Phenylpropanoids & Coumarins & Not annotated \\
\hline Sediment (non-saline) & 6 & 588 & $2 \mathrm{C9H} 6 \mathrm{O} 2$ & Shikimates and Phenylpropanoids & Phenylpropanoids (C6-C3) & Cinnamic acids and derivatives \\
\hline Sediment (non-saline) & 7 & 172 & $1 \mathrm{C} 27 \mathrm{H} 3805$ & Terpenoids & Not annotated & Not annotated \\
\hline Sediment (non-saline) & 8 & 1109 & 9 C19H3405 & Fatty acids & Linear polyketides & Open-chain polyketides \\
\hline Sediment (non-saline) & 9 & 2535 & 1 Not annotated & Not annotated & Not annotated & Not annotated \\
\hline Sediment (non-saline) & 10 & 1508 & $6 \mathrm{C} 12 \mathrm{H} 1806$ & Polyketides & Not annotated & Not annotated \\
\hline Sediment (saline) & 1 & 1261 & $7 \mathrm{C} 27 \mathrm{H} 47 \mathrm{NO} 13$ & Fatty acids & Not annotated & Not annotated \\
\hline Sediment (saline) & 2 & 4220 & 2 Not annotated & Not annotated & Not annotated & Not annotated \\
\hline Sediment (saline) & 3 & 1036 & $1 \mathrm{C} 42 \mathrm{H} 56 \mathrm{O}$ & Terpenoids & Carotenoids (C40) & Carotenoids $(C 40, \beta-\beta)$ \\
\hline Sediment (saline) & 4 & 1511 & 8 C19H35NO9 & Fatty acids & Not annotated & Not annotated \\
\hline Sediment (saline) & 5 & 4698 & $4 \mathrm{C} 21 \mathrm{H} 34 \mathrm{O}$ & Terpenoids & Steroids & Cholane steroids \\
\hline Sediment (saline) & 6 & 3311 & 3 C32H57NO9 & Polyketides & Linear polyketides & Open-chain polyketides \\
\hline Sediment (saline) & 7 & 1253 & 7 C4OH5805 & Terpenoids & Carotenoids (C40) & Carotenoids $(C 40, \beta-\beta)$ \\
\hline Sediment (saline) & 8 & 1286 & $2 \mathrm{C} 21 \mathrm{H} 34 \mathrm{O}$ & Fatty acids & Eicosanoids & Not annotated \\
\hline Sediment (saline) & 9 & 1262 & $3 \mathrm{C} 28 \mathrm{H} 49 \mathrm{NO} 13$ & Fatty acids & Not annotated & Not annotated \\
\hline Sediment (saline) & 10 & 1275 & 0 Not annotated & Not annotated & Not annotated & Not annotated \\
\hline
\end{tabular}


bioRxiv preprint doi: https://doi.org/10.1101/2021.06.04.446988; this version posted November 4, 2021. The copyright holder for this preprint (which was not certified by peer review) is the author/funder. All rights reserved. No reuse allowed without permission.

\section{Shaffer et al. Metabolite-microbe profiles are shaped by the environment}

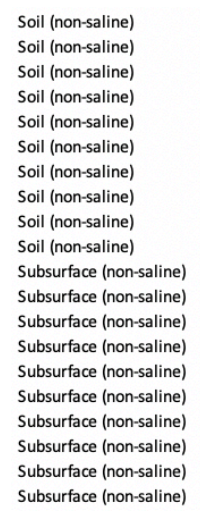

2102 C18H37NO2
10177 C32H52N8O5
2073 C17H19NO2
2217 C13H21CIN2O3
2161 C32H51N5O5
7661 C2OH39NO
2135 C18H18N2O
2288 C11H12N2O2
7420 C13H19FOS2
5153 C2OH22O4
14373 C14H19NO
14208 C8H12O4
42569 Not annotated
17757 C2OH20O
6222 C18H22
27023 C17H32O5
14337 Not annotated
15209 C16H20O2S
$2225 \mathrm{C} 14 \mathrm{H} 21$ NO
9506 C17H18O

Fatty acids
Amino acids and Peptides
Alkaloids
Fatty acids
Amino acids and Peptides
Fatty acids
Alkaloids
Alkaloids
Terpenoids
Shikimates and Phenylpropanoids
Terpenoids
Fatty acids
Not annotated
Terpenoids
Terpenoids
Fatty acids
Not annotated
Terpenoids
Fatty acids
Terpenoids

Fatty Acids and Conjugates

Unsaturated fatty acids

Oligopeptides

Not annotated

Not annotated

Oligopeptides

Fatty amides

Tryptophan alkaloids

Monoterpenoids

Lignans

Sesquiterpenoids

Fatty Acids and Conjugates

Not annotated

Steroids

Steroids

Glycerolipids

Not annotated

Steroids

Fatty amides

Not annotated

Linear peptides

Not annotated

Not annotated

Cyclic peptides

Primary amides

Simple indole alkaloids

Simple indole alkaloids

Not annotated

Dibenzylbutane lignans

Not annotated

Dicarboxylic acids

Not annotated

Not annotated

Estrane steroids

Monoacylglycerols

Not annotated

Not annotated

Phenylalanine-derived alkaloids

Not annotated

$\underline{\text { Table S2. (continued) }}$

\begin{tabular}{|c|c|c|c|c|c|c|}
\hline Environment & Feature rank & Feature ID & Molecular formula & Pathway & Superclass & Class \\
\hline Water (non-saline) & 1 & 14668 & $\mathrm{C} 30 \mathrm{H} 62 \mathrm{O} 16$ & Carbohydrates & Glycerolipids & Not annotated \\
\hline Water (non-saline) & 2 & 14675 & $\mathrm{C} 18 \mathrm{H} 33 \mathrm{~N} 705$ & Alkaloids & Pseudoalkaloids (transamidation) & Not annotated \\
\hline Water (non-saline) & 3 & 14677 & С32H66017 & Carbohydrates & Glycerolipids & Not annotated \\
\hline Water (non-saline) & 4 & 14832 & $\mathrm{C} 19 \mathrm{H} 31 \mathrm{BN} 604$ & Alkaloids & Not annotated & Not annotated \\
\hline Water (non-saline) & 5 & 14725 & $\mathrm{C} 18 \mathrm{H} 38010$ & Fatty acids & Glycerolipids & Diacylglycerols \\
\hline Water (non-saline) & 6 & 14693 & $\mathrm{C} 22 \mathrm{H} 46012$ & Fatty acids & Glycerolipids & Diacylglycerols \\
\hline Water (non-saline) & 7 & 14821 & $\mathrm{C} 14 \mathrm{H} 2807$ & Fatty acids & Not annotated & Not annotated \\
\hline Water (non-saline) & 8 & 14665 & $\mathrm{C} 28 \mathrm{H} 58015$ & Carbohydrates & Glycerolipids & Not annotated \\
\hline Water (non-saline) & 9 & 15877 & Not annotated & Not annotated & Not annotated & Not annotated \\
\hline Water (non-saline) & 10 & 14797 & Not annotated & Not annotated & Not annotated & Not annotated \\
\hline Water (saline) & 1 & 14668 & С30H62O16 & Carbohydrates & Glycerolipids & Not annotated \\
\hline Water (saline) & 2 & 14677 & C32H66O17 & Carbohydrates & Glycerolipids & Not annotated \\
\hline Water (saline) & 3 & 14832 & $\mathrm{C} 19 \mathrm{H} 31 \mathrm{BN} 604$ & Alkaloids & Not annotated & Not annotated \\
\hline Water (saline) & 4 & 14665 & $\mathrm{C} 28 \mathrm{H} 58015$ & Carbohydrates & Glycerolipids & Not annotated \\
\hline Water (saline) & 5 & 14693 & $\mathrm{C} 22 \mathrm{H} 46 \mathrm{O} 12$ & Fatty acids & Glycerolipids & Diacylglycerols \\
\hline Water (saline) & 6 & 14675 & $\mathrm{C} 18 \mathrm{H} 33 \mathrm{~N} 705$ & Alkaloids & Pseudoalkaloids (transamidation) & Not annotated \\
\hline Water (saline) & 7 & 15877 & Not annotated & Not annotated & Not annotated & Not annotated \\
\hline Water (saline) & 8 & 14785 & Not annotated & Not annotated & Not annotated & Not annotated \\
\hline Water (saline) & 9 & 14844 & $\mathrm{C} 26 \mathrm{H} 55 \mathrm{NO} 13$ & Carbohydrates & Aminosugars and aminoglycosides & Not annotated \\
\hline Water (saline) & 10 & 14724 & $\mathrm{C} 24 \mathrm{H} 50013$ & Fatty acids & Glycerolipids & Diacylglycerols \\
\hline
\end{tabular}


bioRxiv preprint doi: https://doi.org/10.1101/2021.06.04.446988; this version posted November 4, 2021. The copyright holder for this preprint (which was not certified by peer review) is the author/funder. All rights reserved. No reuse allowed without permission.

Shaffer et al. Metabolite-microbe profiles are shaped by the environment

Table S3. The top 25 microbially-related metabolites across all environments, ranked based on association with RPCA ordination axes (i.e., global magnitude across all axes). Metabolites in bold font are those also identified to be strongly associated with particular environments from multinomial regression (Table S2), those in bold, underlined font also strongly separated environments in machine-learning analysis (Table S4), and those in bold, underlined, italic font identified as important in all three analyses.

\begin{tabular}{|c|c|c|c|c|}
\hline Feature ID & Molecular formu & Pathway & Superclass & Class \\
\hline 362 & $\mathrm{C} 17 \mathrm{H} 24 \mathrm{O} 3$ & Terpenoids & Sesquiterpenoids & Not annotated \\
\hline 1630 & $\mathrm{C} 9 \mathrm{H} 8 \mathrm{O} 2$ & Shikimates and Phenylpropanoids & Phenylpropanoids (C6-C3) & Cinnamic acids and derivatives \\
\hline 1632 & $\mathrm{C} 9 \mathrm{H} 8 \mathrm{O} 2$ & Shikimates and Phenylpropanoids & Phenolic acids (C6-C1) & Simple phenolic acids \\
\hline 1856 & $\mathrm{C} 22 \mathrm{H} 41 \mathrm{NO}$ & Fatty acids & Fatty amides & Primary amides \\
\hline 3394 & $\mathrm{C} 28 \mathrm{H} 38$ & Terpenoids & Steroids & Vitamin D2 and derivatives \\
\hline 3830 & $\mathrm{C} 28 \mathrm{H} 38$ & Terpenoids & Steroids & Vitamin D2 and derivatives \\
\hline 4561 & $\mathrm{C} 9 \mathrm{H} 6 \mathrm{O} 2$ & Shikimates and Phenylpropanoids & Phenylpropanoids (C6-C3) & Cinnamic acids and derivatives \\
\hline 4949 & C13H10O & Shikimates and Phenylpropanoids & Flavonoids & Chalcones \\
\hline 6974 & $\mathrm{C} 14 \mathrm{H} 24 \mathrm{O} 5$ & Terpenoids & Not annotated & Not annotated \\
\hline 8263 & Not annotated & Not annotated & Not annotated & Not annotated \\
\hline 8273 & Not annotated & Not annotated & Not annotated & Not annotated \\
\hline 12599 & C10H10O2 & Shikimates and Phenylpropanoids & Phenylpropanoids (C6-C3) & Cinnamic acids and derivatives \\
\hline$\overline{13892}$ & $\overline{\mathrm{C} 8 \mathrm{H} 18 \mathrm{O} 5}$ & Fatty acids & $\overrightarrow{\text { Glycerolipids }}$ & Diacylglycerols \\
\hline 14665 & C28H58O15 & Carbohydrates & Glycerolipids & Not annotated \\
\hline 14675 & $\mathrm{C} 18 \mathrm{H} 33 \mathrm{~N} 7 \mathrm{O} 5$ & Alkaloids & Pseudoalkaloids (transamidation) & Not annotated \\
\hline 14680 & $\mathrm{C} 22 \mathrm{H} 35 \mathrm{~N} 5 \mathrm{O} 5$ & Alkaloids & Pseudoalkaloids (transamidation) & Not annotated \\
\hline 15105 & $\mathrm{C} 26 \mathrm{H} 37 \mathrm{NO} 6$ & Terpenoids & Diterpenoids & Tigliane diterpenoids \\
\hline 16345 & Not annotated & Not annotated & Not annotated & Not annotated \\
\hline 22299 & $\mathrm{C} 29 \mathrm{H} 46 \mathrm{O} 2$ & Terpenoids & Meroterpenoids & Prenyl quinone meroterpenoids \\
\hline$\underline{25552}$ & C24H34O2 & Terpenoids & Steroids & Cholane steroids \\
\hline 33591 & $\mathrm{C} 19 \mathrm{H} 30 \mathrm{O} 2$ & Terpenoids & Diterpenoids & Androstane steroids \\
\hline 33598 & $\mathrm{C} 9 \mathrm{H} 8 \mathrm{O} 2$ & Shikimates and Phenylpropanoids & Phenolic acids (C6-C1) & Simple phenolic acids \\
\hline$\underline{34551}$ & C10H17N3O2 & Alkaloids & Histidine alkaloids & Imidazole alkaloids \\
\hline 46387 & $\mathrm{C} 19 \mathrm{H} 35 \mathrm{~N} 7 \mathrm{O} 3 \mathrm{~S}$ & Amino acids and Peptides & Not annotated & Not annotated \\
\hline$\underline{58021}$ & $\mathrm{C} 8 \mathrm{H} 10 \mathrm{~N} 4 \mathrm{O} 2$ & Alkaloids & Pseudoalkaloids (transamidation) & Purine alkaloids \\
\hline
\end{tabular}


bioRxiv preprint doi: https://doi.org/10.1101/2021.06.04.446988; this version posted November 4, 2021. The copyright holder for this preprint (which was not certified by peer review) is the author/funder. All rights reserved. No reuse allowed without permission.

Shaffer et al. Metabolite-microbe profiles are shaped by the environment

Table S4. The top 32 most important metabolites across all metabolites contributing to separation of environments, identified in random forest machine-learning analysis. Metabolites in bold font are those also identified to be strongly associated with particular environments from multinomial regression (Table S2), those in bold, underlined font also identified to be strongly associated with RPCA axes (Fig. 2c, Table S3), and those in bold, underlined, italic font identified as important in all three analyses.

\begin{tabular}{|c|c|c|c|c|}
\hline Feature ID & Molecular formula & Pathway & Superclass & Class \\
\hline 5473 & $\mathrm{C} 20 \mathrm{H} 18 \mathrm{O} 6$ & Shikimates and Phenylpropanoids & Lignans & Not annotated \\
\hline 7792 & $\mathrm{C} 24 \mathrm{H} 36 \mathrm{O} 3$ & Terpenoids & Steroids & Cholane steroids \\
\hline 7811 & $\mathrm{C} 24 \mathrm{H} 36 \mathrm{O} 3$ & Terpenoids & Steroids & Cholane steroids \\
\hline 10836 & $\mathrm{C} 24 \mathrm{H} 45 \mathrm{NO} 3$ & Fatty acids & Fatty amides & $\mathrm{N}$-acyl amines \\
\hline 12599 & $\underline{\mathrm{C} 10 \mathrm{H} 10 \mathrm{O} 2}$ & Shikimates and Phenylpropanoids & Phenylpropanoids (C6-C3) & Cinnamic acids and derivatives \\
\hline$\overline{16072}$ & Not annotated & Not annotated & Not annotated & Not annotated \\
\hline 19163 & $\mathrm{C} 10 \mathrm{H} 14 \mathrm{~N} 6 \mathrm{O} 4$ & Amino acids and Peptides & Small peptides & Dipeptides \\
\hline 21810 & $\mathrm{C} 18 \mathrm{H} 1805$ & Shikimates and Phenylpropanoids & Not annotated & Not annotated \\
\hline$\underline{25552}$ & $\underline{\mathrm{C} 24 \mathrm{H} 34 \mathrm{O} 2}$ & Terpenoids & $\underline{\text { Steroids }}$ & Cholane steroids \\
\hline$\overline{25723}$ & $\overline{\mathrm{C} 18 \mathrm{H} 39 \mathrm{NO} 4}$ & Fatty acids & $\overline{\text { Fatty acyls }}$ & Fatty alcohols \\
\hline 26885 & $\mathrm{C} 11 \mathrm{H} 23 \mathrm{~N} 3 \mathrm{O} 2$ & Alkaloids & Ornithine alkaloids & Polyamines \\
\hline 30382 & $\mathrm{C} 27 \mathrm{H} 38 \mathrm{O}$ & Terpenoids & Steroids & Cholestane steroids \\
\hline 30829 & $\mathrm{C} 27 \mathrm{H} 40 \mathrm{O}$ & Terpenoids & Steroids & Not annotated \\
\hline 31550 & Not annotated & Not annotated & Not annotated & Not annotated \\
\hline 34312 & $\mathrm{C} 24 \mathrm{H} 45 \mathrm{NO} 2$ & Fatty acids & Fatty amides & Not annotated \\
\hline$\underline{34551}$ & $\underline{\mathrm{C} 10 \mathrm{H} 17 \mathrm{~N} 3 \mathrm{O} 2}$ & Alkaloids & Histidine alkaloids & Imidazole alkaloids \\
\hline$\overline{35605}$ & $\overline{\text { Not annotated }}$ & Not annotated & Not annotated & Not annotated \\
\hline 39906 & C27H39NO & Terpenoids & Steroids & Not annotated \\
\hline 40604 & $\mathrm{C} 27 \mathrm{H} 43 \mathrm{NO} 3$ & Fatty acids & Fatty amides & $\mathrm{N}$-acyl amines \\
\hline 42244 & $\mathrm{C} 12 \mathrm{H} 20 \mathrm{~N} 2 \mathrm{O} 4$ & Amino acids and Peptides & Small peptides & Dipeptides \\
\hline 46477 & $\mathrm{C} 17 \mathrm{H} 22 \mathrm{~N} 2 \mathrm{O} 4$ & Alkaloids & Ornithine alkaloids & Not annotated \\
\hline 46951 & $\mathrm{C} 27 \mathrm{H} 47 \mathrm{NO} 5$ & Terpenoids & Steroids & Cholestane steroids \\
\hline 47330 & $\mathrm{C} 8 \mathrm{H} 14 \mathrm{~N} 2$ & Alkaloids & Nicotinic acid alkaloids & Pyridine alkaloids \\
\hline 48540 & $\mathrm{C} 14 \mathrm{H} 18 \mathrm{~N} 2 \mathrm{O}$ & Alkaloids & Tryptophan alkaloids & Simple indole alkaloids \\
\hline 48746 & $\mathrm{C} 17 \mathrm{H} 24 \mathrm{~N} 2 \mathrm{O} 4$ & Alkaloids & Ornithine alkaloids & Not annotated \\
\hline 53313 & $\mathrm{C} 18 \mathrm{H} 16 \mathrm{O} 2$ & Shikimates and Phenylpropanoids & Lignans & Not annotated \\
\hline 53367 & $\mathrm{C} 27 \mathrm{H} 44$ & Terpenoids & Steroids & Cholestane steroids \\
\hline 56133 & $\mathrm{C} 27 \mathrm{H} 42 \mathrm{O}$ & Terpenoids & Steroids & Cholestane steroids \\
\hline$\underline{58021}$ & $\underline{\mathrm{C} 8 \mathrm{H} 10 \mathrm{~N} 4 \mathrm{O} 2}$ & Alkaloids & Pseudoalkaloids (transamidation) & Purine alkaloids \\
\hline 58024 & $\mathrm{C} 8 \mathrm{H} 10 \mathrm{~N} 4 \mathrm{O} 2$ & Alkaloids & Pseudoalkaloids (transamidation) & Purine alkaloids \\
\hline 58025 & $\mathrm{C} 8 \mathrm{H} 10 \mathrm{~N} 4 \mathrm{O} 2$ & Alkaloids & Pseudoalkaloids (transamidation) & Purine alkaloids \\
\hline 58026 & $\mathrm{C} 8 \mathrm{H} 10 \mathrm{~N} 4 \mathrm{O} 2$ & Alkaloids & Pseudoalkaloids (transamidation) & Purine alkaloids \\
\hline
\end{tabular}


Shaffer et al. Metabolite-microbe profiles are shaped by the environment

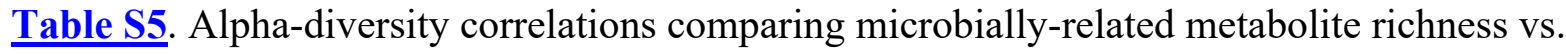

either shotgun metagenomic microbial taxonomic richness, Faith's PD, or weighted Faith's PD.

Values are Spearman correlation coefficients, and p-values were adjusted using the Benjamini-

Hochberg correction.

\begin{tabular}{|c|c|c|c|c|c|c|c|c|}
\hline Environment & $n$ & shotgun metric & $r$ & lower $95 \% \mathrm{Cl}$ & I upper $95 \% \mathrm{Cl}$ & $t$ & $s$ & $p$-value \\
\hline Animal corpus (non-saline) & 3 & richness & $\mathrm{N} / \mathrm{A}$ & $\mathrm{N} / \mathrm{A}$ & N/A & $\mathrm{N} / \mathrm{A}$ & $\mathrm{N} / \mathrm{A}$ & $\mathrm{N} / \mathrm{A}$ \\
\hline Animal corpus (saline) & 44 & richness & 0.39 & 0.11 & 0.62 & 2.74 & N/A & 0.01 \\
\hline Animal distal gut (non-saline) & 139 & richness & 3.43E-04 & -0.17 & 0.17 & 4.01E-03 & N/A & 1 \\
\hline Animal distal gut (saline) & 11 & richness & -0.12 & -0.68 & 0.53 & N/A & 274.06 & 0.7 \\
\hline Animal proximal gut (non-saline) & 5 & richness & -0.21 & -0.93 & 0.84 & N/A & 24.1 & 0.7 \\
\hline Animal proximal gut (saline) & 12 & richness & 0.73 & 0.26 & 0.92 & N/A & 76 & 0.01 \\
\hline Animal secretion (saline) & 12 & richness & 0.05 & -0.55 & 0.62 & N/A & 272 & 0.9 \\
\hline Fungus corpus (non-saline) & 6 & richness & 0.43 & -0.61 & 0.93 & N/A & 20 & 0.4 \\
\hline Plant corpus (non-saline) & 26 & richness & 0.74 & 0.49 & 0.88 & N/A & 750.63 & 0.001 \\
\hline Plant surface (non-saline) & 13 & richness & -0.36 & -0.77 & 0.26 & N/A & 494 & 0.2 \\
\hline Plant surface (saline) & 0 & richness & $N / A$ & N/A & N/A & N/A & N/A & N/A \\
\hline Sediment (non-saline) & 31 & richness & 0.42 & 0.07 & 0.67 & 2.47 & N/A & 0.05 \\
\hline Sediment (saline) & 31 & richness & 0.27 & -0.09 & 0.57 & 1.52 & N/A & 0.1 \\
\hline Soil (non-saline) & 78 & richness & 0.06 & -0.16 & 0.28 & 0.54 & N/A & 0.6 \\
\hline Soil (saline) & 0 & richness & N/A & N/A & N/A & N/A & N/A & N/A \\
\hline Subsurface (non-saline) & 3 & richness & N/A & N/A & N/A & N/A & N/A & N/A \\
\hline Surface (saline) & 2 & richness & N/A & N/A & N/A & N/A & N/A & N/A \\
\hline Water (non-saline) & 8 & richness & 0.24 & -0.56 & 0.81 & 0.6 & N/A & 0.6 \\
\hline Water (saline) & 30 & richness & 0.57 & 0.26 & 0.77 & 3.65 & N/A & 0.01 \\
\hline Animal corpus (non-saline) & 3 & Faith's PD & N/A & N/A & N/A & N/A & N/A & N/A \\
\hline Animal corpus (saline) & 44 & Faith's PD & 0.29 & -0.01 & 0.54 & 1.98 & N/A & 0.05 \\
\hline Animal distal gut (non-saline) & 139 & Faith's PD & 0.02 & -0.15 & 0.18 & 0.19 & N/A & 0.8 \\
\hline Animal distal gut (saline) & 11 & Faith's PD & -0.05 & -0.64 & 0.58 & N/A & 230 & 0.9 \\
\hline Animal proximal gut (non-saline) & 5 & Faith's PD & 0.1 & -0.87 & 0.91 & N/A & 18 & 0.9 \\
\hline Animal proximal gut (saline) & 12 & Faith's PD & 0.72 & 0.23 & 0.92 & N/A & 80 & 0.01 \\
\hline Animal secretion (saline) & 12 & Faith's PD & 0.17 & -0.46 & 0.69 & N/A & 238 & 0.6 \\
\hline Fungus corpus (non-saline) & 6 & Faith's PD & 0.43 & -0.61 & 0.93 & N/A & 20 & 0.4 \\
\hline Plant corpus (non-saline) & 26 & Faith's PD & 0.74 & 0.49 & 0.88 & N/A & 760 & 0.001 \\
\hline Plant surface (non-saline) & 13 & Faith's PD & -0.41 & -0.79 & 0.2 & N/A & 512 & 0.2 \\
\hline Plant surface (saline) & 0 & Faith's PD & N/A & N/A & N/A & N/A & N/A & N/A \\
\hline Sediment (non-saline) & 31 & Faith's PD & 0.44 & 0.1 & 0.69 & 2.65 & N/A & 0.05 \\
\hline Sediment (saline) & 31 & Faith's PD & 0.09 & -0.28 & 0.43 & 0.47 & N/A & 0.6 \\
\hline Soil (non-saline) & 78 & Faith's PD & 8.73E-04 & -0.22 & 0.22 & 7.61E-03 & N/A & 1 \\
\hline Soil (saline) & 0 & Faith's PD & N/A & N/A & N/A & N/A & N/A & N/A \\
\hline Subsurface (non-saline) & 3 & Faith's PD & N/A & N/A & N/A & N/A & N/A & N/A \\
\hline Surface (saline) & 2 & Faith's PD & N/A & N/A & N/A & N/A & N/A & N/A \\
\hline Water (non-saline) & 8 & Faith's PD & -0.24 & -0.81 & 0.56 & -0.6 & N/A & 0.6 \\
\hline Water (saline) & 30 & Faith's PD & 0.6 & 0.31 & 0.79 & 4.01 & N/A & 0.001 \\
\hline Animal corpus (non-saline) & 3 & weighted Faith's PD & N/A & N/A & N/A & N/A & N/A & N/A \\
\hline Animal corpus (saline) & 44 & weighted Faith's PD & -0.13 & -0.41 & 0.17 & -0.87 & N/A & 0.4 \\
\hline Animal distal gut (non-saline) & 139 & weighted Faith's PD & 0.05 & -0.11 & 0.22 & 0.64 & N/A & 0.5 \\
\hline Animal distal gut (saline) & 11 & weighted Faith's PD & -0.39 & -0.81 & 0.29 & N/A & 306 & 0.2 \\
\hline Animal proximal gut (non-saline) & 5 & weighted Faith's PD & 0.1 & -0.87 & 0.91 & N/A & 18 & 0.9 \\
\hline Animal proximal gut (saline) & 12 & weighted Faith's PD & 0.53 & -0.08 & 0.85 & N/A & 134 & 0.08 \\
\hline Animal secretion (saline) & 12 & weighted Faith's PD & 0.31 & -0.34 & 0.76 & N/A & 198 & 0.3 \\
\hline Fungus corpus (non-saline) & 6 & weighted Faith's PD & 0.6 & -0.44 & 0.95 & N/A & 14 & 0.2 \\
\hline Plant corpus (non-saline) & 26 & weighted Faith's PD & 0.46 & 0.07 & 0.72 & N/A & 1585 & 0.05 \\
\hline Plant surface (non-saline) & 13 & weighted Faith's PD & -0.45 & -0.81 & 0.15 & N/A & 528 & 0.12 \\
\hline Plant surface (saline) & 0 & weighted Faith's PD & N/A & N/A & N/A & N/A & N/A & N/A \\
\hline Sediment (non-saline) & 31 & weighted Faith's PD & 0.35 & 0 & 0.63 & 2.02 & N/A & 0.05 \\
\hline Sediment (saline) & 31 & weighted Faith's PD & -0.19 & -0.51 & 0.17 & -0.105 & N/A & 0.3 \\
\hline Soil (non-saline) & 78 & weighted Faith's PD & $-6.16 \mathrm{E}+03$ & -0.23 & 0.22 & -0.05 & N/A & 1 \\
\hline Soil (saline) & 0 & weighted Faith's PD & N/A & N/A & N/A & N/A & N/A & N/A \\
\hline Subsurface (non-saline) & 3 & weighted Faith's PD & N/A & N/A & N/A & N/A & N/A & N/A \\
\hline Surface (saline) & 2 & weighted Faith's PD & N/A & N/A & N/A & $\mathrm{N} / \mathrm{A}$ & N/A & N/A \\
\hline Water (non-saline) & 8 & weighted Faith's PD & 0.12 & -0.64 & 0.76 & 0.29 & N/A & 0.8 \\
\hline Water (saline) & 30 & weighted Faith's PD & 0.42 & 0.08 & 0.68 & 2.48 & $\mathrm{~N} / \mathrm{A}$ & 0.05 \\
\hline
\end{tabular}


Shaffer et al. Metabolite-microbe profiles are shaped by the environment

Table S6. Mantel test results comparing data layers generated for the EMP500 samples. Note the strong relationships between the metabolomics data (i.e., LC-MS/MS and GC-MS) and the sequence data from Bacteria and Archaea (i.e., shotgun metagenomics, 16S, and full-length rRNA operon) as compared to between metabolomics data and sequence data from eukaryotes (i.e., 18S and ITS), as well as the strong relationships between difference sequence data from Bacteria and Archaea (rho $>0.2$ in bolded font; $>0.4$ in bolded, italics; $>0.5$ additionally underlined).

\begin{tabular}{|c|c|c|c|c|}
\hline Dataset 1 & Dataset 2 & $n$ & Spearman rho & p-value \\
\hline \multirow[t]{7}{*}{ LC-MS/MS } & GC-MS & 401 & 0.13 & 0.001 \\
\hline & Metagenomics (taxa) & 454 & 0.43 & 0.001 \\
\hline & Metagenomics (function) & 378 & 0.24 & 0.001 \\
\hline & $16 S$ & 477 & 0.27 & 0.001 \\
\hline & $18 S$ & 356 & 0.06 & 0.034 \\
\hline & ITS & 359 & 0.08 & 0.001 \\
\hline & full-length rRNA operon & 181 & 0.34 & 0.001 \\
\hline \multirow[t]{6}{*}{ GC-MS } & Metagenomics (taxa) & 331 & 0.07 & 0.002 \\
\hline & Metagenomics (function) & 279 & 0.05 & 0.029 \\
\hline & $16 S$ & 349 & 0.22 & 0.001 \\
\hline & $18 S$ & 284 & -0.01 & 0.641 \\
\hline & ITS & 258 & 0.02 & 0.404 \\
\hline & full-length rRNA operon & 168 & 0.11 & 0.001 \\
\hline \multirow[t]{5}{*}{ Metagenomics (taxa) } & Metagenomics (function) & 508 & 0.24 & 0.001 \\
\hline & $16 S$ & 538 & $\underline{0.51}$ & 0.001 \\
\hline & $18 S$ & 378 & -0.04 & 0.159 \\
\hline & ITS & 408 & 0.05 & 0.023 \\
\hline & full-length rRNA operon & 235 & 0.48 & 0.001 \\
\hline \multirow[t]{4}{*}{ Metagenomics (function) } & $16 S$ & 449 & 0.11 & 0.001 \\
\hline & $18 S$ & 305 & 0.12 & 0.001 \\
\hline & ITS & 337 & 0.14 & 0.001 \\
\hline & full-length rRNA operon & 219 & 0.21 & 0.001 \\
\hline \multirow[t]{3}{*}{$16 S$} & $18 \mathrm{~S}$ & 430 & 0.04 & 0.117 \\
\hline & ITS & 447 & 0.09 & 0.001 \\
\hline & full-length rRNA operon & 215 & 0.51 & 0.001 \\
\hline \multirow[t]{2}{*}{$18 \mathrm{~S}$} & ITS & 387 & 0.20 & 0.001 \\
\hline & full-length rRNA operon & 174 & 0.06 & 0.06 \\
\hline ITS & full-length rRNA operon & 166 & 0.09 & 0.001 \\
\hline
\end{tabular}


Shaffer et al. Metabolite-microbe profiles are shaped by the environment

\section{SUPPLEMENTARY NOTES}

\section{Permits for sample collection.}

For samples from Costa Rica, permits were granted by the Institutional Biodiversity Commission of the University of Costa Rica (UCR, resolution number 055-2016) and authorized by the Organization for Tropical Studies (OTS) and the Central Pacific Conservation Area (ACOPAC) of the Ministry of Energy and the Environment (MINAE), Costa Rican government, under UCR project B6-656.

\section{Data availability.}

The mass spectrometry method and data (.RAW and .mzML) were deposited on the MassIVE public repository and are available under the dataset accession number MSV000083475. The processing files were also added to the deposition (updates/2019-08-

21_lfnothias_7cc0af40/other/1908_EMPv2_INN/). GNPS molecular networking job is available at https://gnps.ucsd.edu/ProteoSAFe/status.jsp?task=929ce9411f684cf8abd009670b293a33 and was also performed in analogue mode https://gnps.ucsd.edu/ProteoSAFe/status.jsp?task=fafdbfc058184c2b8c87968a7c56d7aa. The DEREPLICATOR jobs can be accessed here: https:/gnps.ucsd.edu/ProteoSAFe/status.jsp?task=ee40831bcc314bda928886964d853a52 and https://gnps.ucsd.edu/ProteoSAFe/status.jsp?task=1fafd4d4fe7e47dd9dd0b3d8bb0e6606. The SIRIUS results are available on the GitHub repository ['emp/data/metabolomics/FBMN/SIRIUS']. The notebooks for metabolomics data preparation and microbially-related molecules establishment are available on this repository 
Shaffer et al. Metabolite-microbe profiles are shaped by the environment

(https://github.com/lfnothias/emp_metabolomic). Amplicon and shotgun metagenomic sequence data are submitted to the European Nucleotide Archive under Project: PRJEB42019

(https://www.ebi.ac.uk/ena/browser/view/PRJEB42019). Raw and demultiplexed amplicon and shotgun sequence data, the feature-table for full-length rRNA operon analysis, feature-tables for LC-MS/MS classical molecular networking and feature-based molecular networking, and the feature-table for GC-MS molecular networking data are available for download and analysis through Qiita at qiita.ucsd.edu (study: 13114).

\section{References.}

1. Thompson, L. R. et al. A communal catalogue reveals Earth's multiscale microbial diversity. Nature 551, 457-463 (2017).

2. Thompson, L. et al. EMP Sample Submission Guide v1 (protocols.io.pfqdjmw). protocols.io (2018) doi:10.17504/protocols.io.pfqdjmw.

3. Yilmaz, P. et al. Minimum information about a marker gene sequence (MIMARKS) and minimum information about any (x) sequence $(\mathrm{MIxS})$ specifications. Nat. Biotechnol. 29, 415-420 (2011).

4. Gonzalez, A. et al. Qiita: rapid, web-enabled microbiome meta-analysis. Nat. Methods 15, 796-798 (2018).

5. Chambers, M. C. et al. A cross-platform toolkit for mass spectrometry and proteomics. Nat. Biotechnol. 30, 918-920 (2012).

6. Pluskal, T., Castillo, S., Villar-Briones, A. \& Oresic, M. MZmine 2: modular framework for processing, visualizing, and analyzing mass spectrometry-based molecular profile data. BMC Bioinformatics 11, 395 (2010). 
Shaffer et al. Metabolite-microbe profiles are shaped by the environment

7. Schmid, R. et al. Ion Identity Molecular Networking in the GNPS Environment. Cold Spring Harbor Laboratory 2020.05.11.088948 (2020) doi:10.1101/2020.05.11.088948.

8. Du, X., Smirnov, A., Pluskal, T., Jia, W. \& Sumner, S. Metabolomics Data Preprocessing Using ADAP and MZmine 2. Methods Mol. Biol. 2104, 25-48 (2020).

9. Nothias, L.-F. et al. Feature-based molecular networking in the GNPS analysis environment. Nat. Methods 17, 905-908 (2020).

10. Wang, M. et al. Sharing and community curation of mass spectrometry data with Global Natural Products Social Molecular Networking. Nat. Biotechnol. 34, 828-837 (2016).

11. Mohimani, H. et al. Dereplication of peptidic natural products through database search of mass spectra. Nat. Chem. Biol. 13, 30-37 (2017).

12. Mohimani, H. et al. Dereplication of microbial metabolites through database search of mass spectra. Nat. Commun. 9, 4035 (2018).

13. Dührkop, K. et al. SIRIUS 4: a rapid tool for turning tandem mass spectra into metabolite structure information. Nat. Methods 16, 299-302 (2019).

14. Böcker, S., Letzel, M. C., Lipták, Z. \& Pervukhin, A. SIRIUS: decomposing isotope patterns for metabolite identification. Bioinformatics 25, 218-224 (2009).

15. Böcker, S. \& Dührkop, K. Fragmentation trees reloaded. J. Cheminform. 8, 5 (2016).

16. Ludwig, M. et al. Database-independent molecular formula annotation using Gibbs sampling through ZODIAC. Nature Machine Intelligence 2, 629-641 (2020).

17. Dührkop, K., Shen, H., Meusel, M., Rousu, J. \& Böcker, S. Searching molecular structure databases with tandem mass spectra using CSI:FingerID. Proc. Natl. Acad. Sci. U. S. A. 112, $12580-12585$ (2015). 
Shaffer et al. Metabolite-microbe profiles are shaped by the environment

18. Dührkop, K. et al. Systematic classification of unknown metabolites using high-resolution fragmentation mass spectra. Nat. Biotechnol. (2020) doi:10.1038/s41587-020-0740-8.

19. Kim, H. et al. NPClassifier: A Deep Neural Network-Based Structural Classification Tool for Natural Products. J. Nat. Prod. (2021) doi: 10.1021/acs.jnatprod.1c00399

20. van Santen, J. A. et al. The Natural Products Atlas: An Open Access Knowledge Base for Microbial Natural Products Discovery. ACS Cent Sci 5, 1824-1833 (2019).

21. Kautsar, S. A. et al. MIBiG 2.0: a repository for biosynthetic gene clusters of known function. Nucleic Acids Res. 48, D454-D458 (2020).

22. Bolyen, E. et al. Reproducible, interactive, scalable and extensible microbiome data science using QIIME 2. Nat. Biotechnol. 37, 852-857 (2019).

23. Martino, C. et al. A Novel Sparse Compositional Technique Reveals Microbial Perturbations. mSystems 4, (2019).

24. Morton, J. T. et al. Establishing microbial composition measurement standards with reference frames. Nat. Commun. 10, 2719 (2019).

25. Vázquez-Baeza, Y., Pirrung, M., Gonzalez, A. \& Knight, R. EMPeror: a tool for visualizing high-throughput microbial community data. Gigascience 2, 16 (2013).

26. Fedarko, M. W. et al. Visualizing 'omic feature rankings and log-ratios using Qurro. NAR Genom Bioinform 2, lqaa023 (2020).

27. Wilkinson, L. Ggplot2: Elegant graphics for data analysis by WICKHAM, H. Biometrics 67, $678-679$ (2011).

28. Team, R. C. \& Others. R: A language and environment for statistical computing. (2013).

29. Aksenov, A. A. et al. Auto-deconvolution and molecular networking of gas chromatographymass spectrometry data. Nat. Biotechnol. 39, 169-173 (2021). 
Shaffer et al. Metabolite-microbe profiles are shaped by the environment

30. Marotz, L. et al. Earth Microbiome Project (EMP) high throughput (HTP) DNA extraction protocol v1 (protocols.io.pdmdi46). protocols.io (2018) doi:10.17504/protocols.io.pdmdi46.

31. Marotz, C. et al. DNA extraction for streamlined metagenomics of diverse environmental samples. Biotechniques 62, 290-293 (2017).

32. Minich, J. J. et al. KatharoSeq Enables High-Throughput Microbiome Analysis from LowBiomass Samples. mSystems 3, (2018).

33. Minich, J. J. et al. Quantifying and Understanding Well-to-Well Contamination in Microbiome Research. mSystems 4, (2019).

34. Shaffer, J. P. et al. A comparison of DNA/RNA extraction protocols for high-throughput sequencing of microbial communities. Biotechniques 70, 149-159 (2021).

35. Minich, J. J. et al. High-Throughput Miniaturized 16S rRNA Amplicon Library Preparation Reduces Costs while Preserving Microbiome Integrity. mSystems 3, (2018).

36. Karst, S. M. et al. High-accuracy long-read amplicon sequences using unique molecular identifiers with Nanopore or PacBio sequencing. Nat. Methods 18, 165-169 (2021).

37. Greg, J. et al. EMP 16S Illumina Amplicon Protocol v1 (protocols.io.nuudeww). protocols.io (2018) doi:10.17504/protocols.io.nuudeww.

38. Caporaso, J. G. et al. Global patterns of $16 \mathrm{~S}$ rRNA diversity at a depth of millions of sequences per sample. Proc. Natl. Acad. Sci. U. S. A. 108 Suppl 1, 4516-4522 (2011).

39. Caporaso, J. G. et al. Ultra-high-throughput microbial community analysis on the Illumina HiSeq and MiSeq platforms. ISME J. 6, 1621-1624 (2012).

40. Parada, A. E., Needham, D. M. \& Fuhrman, J. A. Every base matters: assessing small subunit rRNA primers for marine microbiomes with mock communities, time series and global field samples. Environ. Microbiol. 18, 1403-1414 (2016). 
Shaffer et al. Metabolite-microbe profiles are shaped by the environment

41. Apprill, A., McNally, S., Parsons, R. \& Weber, L. Minor revision to V4 region SSU rRNA 806R gene primer greatly increases detection of SAR11 bacterioplankton. Aquat. Microb. Ecol. 75, 129-137 (2015).

42. Quince, C., Lanzen, A., Davenport, R. J. \& Turnbaugh, P. J. Removing noise from pyrosequenced amplicons. BMC Bioinformatics 12, 38 (2011).

43. Walters, W. et al. Improved Bacterial 16S rRNA Gene (V4 and V4-5) and Fungal Internal Transcribed Spacer Marker Gene Primers for Microbial Community Surveys. mSystems 1, (2016).

44. Linda et al. EMP 18S Illumina Amplicon Protocol v1 (protocols.io.nuvdew6). protocols.io (2018) doi:10.17504/protocols.io.nuvdew6.

45. Amaral-Zettler, L. A., McCliment, E. A., Ducklow, H. W. \& Huse, S. M. A method for studying protistan diversity using massively parallel sequencing of V9 hypervariable regions of small-subunit ribosomal RNA genes. PLoS One 4, e6372 (2009).

46. Stoeck, T. et al. Multiple marker parallel tag environmental DNA sequencing reveals a highly complex eukaryotic community in marine anoxic water. Mol. Ecol. 19 Suppl 1, 21-31 (2010).

47. Vestheim, H. \& Jarman, S. N. Blocking primers to enhance PCR amplification of rare sequences in mixed samples - a case study on prey DNA in Antarctic krill stomachs. Front. Zool. 5, 12 (2008).

48. Dylan P, S. et al. EMP ITS Illumina Amplicon Protocol v1 (protocols.io.pa7dihn). protocols.io (2018) doi:10.17504/protocols.io.pa7dihn. 
Shaffer et al. Metabolite-microbe profiles are shaped by the environment

49. White, T. et al. Amplification and direct sequencing of fungal ribosomal RNA genes for phylogenetics. PCR protocols: a guide to methods and applications. https://www.scienceopen.com/document?vid=36d59e39-6250-4a7f-b5fe-7155abbb4e03.

50. Hoggard, M. et al. Characterizing the Human Mycobiota: A Comparison of Small Subunit rRNA, ITS1, ITS2, and Large Subunit rRNA Genomic Targets. Front. Microbiol. 9, 2208 (2018).

51. Bokulich, N. A. \& Mills, D. A. Improved selection of internal transcribed spacer-specific primers enables quantitative, ultra-high-throughput profiling of fungal communities. Appl. Environ. Microbiol. 79, 2519-2526 (2013).

52. Klindworth, A. et al. Evaluation of general 16S ribosomal RNA gene PCR primers for classical and next-generation sequencing-based diversity studies. Nucl. Acids Res. 41, e1 (2012).

53. Hunt, D. E. et al. Evaluation of 23S rRNA PCR primers for use in phylogenetic studies of bacterial diversity. Appl. Environ. Microbiol. 72, 2221-2225 (2006).

54. Amir, A. et al. Deblur rapidly resolves single-nucleotide community sequence patterns. mSystems 2:e0191-16 (2017).

55. McDonald, D. et al. An improved Greengenes taxonomy with explicit ranks for ecological and evolutionary analysis of bacteria and archaea. ISME J. 6, 610-618 (2012).

56. Janssen, S. et al. Phylogenetic placement of exact amplicon sequences improves associations with clinical information. mSystems 3:e0021-18 (2018).

57. Bokulich, N. A. et al. Optimizing taxonomic classification of marker-gene amplicon sequences with QIIME 2's q2-feature-classifier plugin. Microbiome 6, 90 (2018). 
Shaffer et al. Metabolite-microbe profiles are shaped by the environment

58. Yilmaz, P. et al. The SILVA and "All-species Living Tree Project (LPT)" taxonomic frameworks. Nucl. Acids Res. 42, D643-D648 (2014).

59. Nilsson, R. H. et al. 2018. The UNITE database for molecular identification of fungi: handling dark taxa and parallel taxonomic classifications. Nucl. Acids Res. 47, D259-D264. (2018).

60. Rognes, T., Flouri, T., Nichols, B., Quince, C., and Mahé, F. Vsearch: a versatile open source tool for metagenomics. PeerJ, 4:e2584 (2016).

61. Sanders, J. G. et al. Optimizing sequencing protocols for leaderboard metagenomics by combining long and short reads. Genome Biol. 20, 226 (2019).

62. Glenn, T. C. et al. Adapterama I: Universal stubs and primers for 384 unique dual-indexed or 147,456 combinatorially-indexed Illumina libraries (iTru \& iNext). bioRxiv 049114 (2019) doi:10.1101/049114.

63. Didion, J. P., Martin, M. \& Collins, F. S. Atropos: specific, sensitive, and speedy trimming of sequencing reads. PeerJ 5, e3720 (2017).

64. Zhu, Q. et al. Phylogenomics of 10,575 genomes reveals evolutionary proximity between domains Bacteria and Archaea. Nat. Commun. 10, 5477 (2019).

65. Pennell, M. W. et al. geiger v2.0: an expanded suite of methods for fitting macroevolutionary models to phylogenetic trees. Bioinformatics 30, 2216-2218 (2014).

66. Swenson, N. G. Functional and Phylogenetic Ecology in R. (Springer, New York, NY, 2014). doi:10.1007/978-1-4614-9542-0.

67. Makowski, D., et al. Methods and Algorithms for Correlation Analysis in R. Journal of Open Source Software, 5, 2306 (2019).

68. Kuhn, M. Classification and Regression Training [R package caret version 6.0-86]. (2020). 
Shaffer et al. Metabolite-microbe profiles are shaped by the environment

69. Morton, J. T. et al. Learning representations of microbe-metabolite interactions. Nat.

Methods 16, 1306-1314 (2019).

\section{Earth Microbiome Project 500 (EMP500) Consortium*}

Lars T. Angenant ${ }^{1}$, Alison M. Berry ${ }^{2}$, Leonora S. Bittleston ${ }^{3}$, Dan Distel ${ }^{4}$, Peter R. Girguis ${ }^{5}$,

Gary M. King ${ }^{6}$, Aurora MacRae-Crerar ${ }^{7}$, Gregory Mayer $^{8}$, Katherine D. McMahon ${ }^{9}$, Jessica L.

Metcalf $^{10}$, David Myrold ${ }^{11}$, Brian Palenik ${ }^{12}$, Forest Rohwer ${ }^{13}$, Stuart A. Sandin ${ }^{12}$, Steven K.

Schmidt $^{14}$, Jennifer E. Smith ${ }^{12}$, Karen Tait ${ }^{15}$, Yael Tucker ${ }^{16}$

${ }^{1}$ University of Tübingen, Tübingen, Germany. ${ }^{2}$ University of California, Davis, Davis, California, USA. ${ }^{3}$ Massachusetts Institute of Technology, Cambridge, Massachusetts, USA.

${ }^{4}$ Northeastern University, Boston, Massachusetts, USA. ${ }^{5}$ Harvard University, Cambridge, Massachusetts, USA. ${ }^{6}$ Louisiana State University, Baton Rouge, Louisiana, USA. ${ }^{7}$ University of Pennsylvania, Philadelphia, Pennsylvania, USA. ${ }^{8}$ Texas Technology University, Lubbock, Texas, USA. ${ }^{9}$ University of Wisconsin, Madison, Wisconsin, USA. ${ }^{10}$ Colorado State University, Fort Collins, Colorado, USA. ${ }^{11}$ Oregon State University, Corvallis, Oregon, USA. ${ }^{12}$ University of California, San Diego, La Jolla, California, USA. ${ }^{13}$ San Diego State University, San Diego, California, USA. ${ }^{14}$ University of Colorado, Boulder, Colorado, USA. ${ }^{15}$ Plymouth Marine

Laboratory, Plymouth, United Kingdom. ${ }^{16}$ National Energy Technology Laboratory, USA. *Listed members are EMP500 principal investigators who contributed samples but did not contribute directly to this paper. 